\title{
Reinforcer Quality and Self-Control
}

by

Heather L. Peters

\author{
A thesis \\ submitted to the Victoria University of Wellington \\ in fulfilment of the \\ requirements for the degree of \\ Doctor of Philosophy \\ in Psychology
}

Victoria University of Wellington

2005 


\section{ACKNOWLEDGEMENTS}

I want to acknowledge the hours of truly patient assistance provided by my supervisors David Harper and Maree Hunt. Appreciation is also extended to Randy Grace for his help with model fitting. Thanks goes also to Victoria University of Wellington, which supplied me with scholarships while I was studying. As well, I want to thank Richard Moore for keeping me supplied with rats and coffee. I also owe a debt of thanks to all of the $7^{\text {th }}$ Floor Lab members for their sympathy during all the repeats. And finally, I want to thank my husband and sons for their unending support without whom the journey that was this thesis would never have begun. 
CONTENTS

LIST OF TABLES $\quad$ V V

LIST OF FIGURES vi

$\begin{array}{ll}\text { ABSTRACT } & 1\end{array}$

GENERAL INTRODUCTION 3

Ethanol Consumption and Drug Abuse 4

Quantifying Choice

Concurrent Schedules and the Generalised Matching Law 7

The Concatenated Matching Law 11

$\begin{array}{ll}\text { Self-Control Paradigms } & 12\end{array}$

The Contextual Choice Model 23

EXPERIMENT 1: REINFORCER QUALITY AND RESPONSE DISTRIBUTION

IN A CONCURRENT CHAINS PROCEDURE

$\begin{array}{ll}\text { Introduction } & 26\end{array}$

$\begin{array}{ll}\text { Method } & 32\end{array}$

$\begin{array}{ll}\text { Results and Discussion } & 38\end{array}$

EXPERIMENT 2: ALTERNATIVE SELF-CONTROL PARADIGMS

$\begin{array}{ll}\text { Introduction } & 60\end{array}$

$\begin{array}{ll}\text { Method } & 67\end{array}$

$\begin{array}{ll}\text { Results } & 74\end{array}$

$\begin{array}{ll}\text { Discussion } & 84\end{array}$

EXPERIMENT 3: MODIFICATION OF THE MN - ML PROCEDURE

$\begin{array}{ll}\text { Introduction } & 87\end{array}$

$\begin{array}{ll}\text { Method } & 89\end{array}$

$\begin{array}{ll}\text { Results and Discussion } & 94\end{array}$ 
EXPERIMENT 4: CONCURRENT ACCESS TO ETHANOL SUCROSE AND

PLAIN SUCROSE

$\begin{array}{ll}\text { Introduction } & 110\end{array}$

$\begin{array}{ll}\text { Method } & 113\end{array}$

$\begin{array}{ll}\text { Results and Discussion } & 115\end{array}$

$\begin{array}{ll}\text { GENERAL DISCUSSION } & 121\end{array}$

$\begin{array}{ll}\text { APPENDIX } & 132\end{array}$

$\begin{array}{ll}\text { REFERENCES } & 134\end{array}$ 


\section{LIST OF TABLES}

Table 1.1 Condition order and terminal link delays to reinforcer delivery for Phase I and Phase II

Table 2.1 Parameters obtained by fitting the GCML to the data from Phase I and

Phase II

Table 2.2 Parameters obtained by fitting CCM to the data from Phase I and

Phase II

Table 3.1 Ethanol water and plain water consumption by the pre-exposed rats

For the final 24-hr free access period

Table 3.2 Ethanol consumption $(\mathrm{g} / \mathrm{kg} / \mathrm{hr})$ for individual subjects

Table 4.1 Order of delay conditions for each subject in Experiment 3

Table 4.2 Individual ethanol consumption $(\mathrm{g} / \mathrm{kg} / \mathrm{hr})$ for each delay condition

Table 4.3 Derived values for bias and sensitivity to the amount ratio

Table 4.4 Obtained slopes for each delay ratio from the re-analysis of White and Pipe (1987)

Table 4.5 Free parameter values derived from fitting Equation 15 to the data from Experiment 3

Table 5.1 Order of conditions and location of the ethanol sucrose and the plain sucrose for each condition

Table 5.2 The average amount of ethanol $(\mathrm{g} / \mathrm{kg} / \mathrm{hr})$ consumed by each subject for the last five sessions of each condition

Table 5.3 Parameters obtained from the GML analysis of the results 


\section{LIST OF FIGURES}

Figure 1.1 Concurrent chains procedure

Figure 1.2 Diagram of the hyperbolic discounting functions for two unequal magnitude reinforcers

Figure 2.1 Schematic diagram of the front wall of the operant chamber

Figure 2.2 Diagram of the concurrent-chains self-control paradigm

Figure 2.3 The average proportion of responses made on the lever associated with the impulsive alternative during Phase I

Figure 2.4 The average proportion of impulsive responses made by each

subject

Figure 2.5 The average proportion of SI choices made by each subject during

Phase I

Figure 2.6 The average proportion of SI choices made by each subject during

Phase II

Figure 2.7 Derived values for the sensitivity parameters

Figure 2.8 Average proportions and sensitivity ratios for each subject

Figure 2.9 The derived values for sensitivity to reinforcer amount and for sensitivity to the reinforcer delay ratio

Figure 2.10 The proportion of SI choices made by each subject in Phase II

Figure 3.1 The novel MN - ML self-control paradigm developed for this

thesis

Figure 3.2 Average proportion of $\mathrm{MN}$ choices made by individual subjects

for Phase II

Figure 3.3 The average proportion of $\mathrm{MN}$ choices made by individual subjects for each delay condition 
Figure 3.5 Log bias for each subject

Figure 4.1 Contingency control graphs for each subject

Figure 4.2 The average proportion of $\mathrm{MN}$ responses made by each subject for each delay condition

Figure 4.3 Individual subject graphs where the log response ratios are plotted as a function of the $\log$ of the amount ratios

Figure 4.4 Choice proportions predicted by the CCM for several different scaling parameter values for Experiment 3

Figure 5.1 Logarithms of the obtained behaviour plotted as a function of the logarithms of the obtained reinforcers 


\section{ABSTRACT}

Self-control has been extensively studied using procedures in which subjects chose between two reinforcer alternatives. Traditionally, one of those alternatives delivers a small reinforcer after a short delay (SI), the other, a larger reinforcer after a long delay (LD). Choosing the SI is defined as impulsivity as it requires forfeit of the larger reinforcer; and choosing the $\mathrm{LD}$ is termed self-control. Four experiments were conducted to examine behaviour using non-human animal analogues of self-control situations. The subjects used for all four experiments were Norway-hooded rats. Experiment 1 used an SI - LD self-control paradigm to examine the effect of manipulating reinforcer quality on response distribution. Findings were that behaviour became more impulsive as the delay ratio became more extreme and this tendency was more systematic when different quality reinforcers were used for the SI and LD alternatives. Experiments 2 and 3 introduced a novel self-control paradigm designed as an analogue of choice situations in which individuals choose between two competing immediately available reinforcers each associated with a different delayed reinforcer. The procedure used was a concurrent-chains schedule that delivered primary reinforcement in the initial and the terminal links. The initial reinforcers were of equal amount and unequal quality; the terminal reinforcers were of unequal amount and equal quality. An impulsive choice was defined as choosing the alternative that delivered the most-valuable reinforcer in the initial link and the leastvalued reinforcer in the terminal link. A self-controlled choice was defined as choosing the alternative that delivered the least-valuable reinforcer in the initial link and the most-valuable reinforcer in the terminal link. The results indicated that behaviour was more self-controlled when the terminal reinforcer quality was ethanol solution and increasing the delay between the initial and terminal links increased 
subjects' responding on the impulsive choice. Behaviour allocation in Experiment 3 was well described by the Contextual Choice Model (Grace, 1994) when the temporal context scaling parameter $(k)$ was allowed to vary. Subjects that were relatively more impulsive had lower derived $k$ values. The final experiment presented the subjects from Experiment 3 with concurrent variable interval (VI) VI schedules in which one alternative delivered plain-sucrose solution and the other ethanol-sucrose solution. Preference measures obtained from Experiment 4 were negatively correlated with the values obtained for the scaling parameter in Experiment 3, indicating that subjects which were more impulsive in the $\mathrm{MN}$ - ML paradigm had a stronger preference for ethanol. In summary, findings indicate that reinforcer quality may change the discriminability of reinforcer alternatives; and the influence of reinforcer quality on response allocation is well described by quantitative models based on the Matching Law. 


\section{REINFORCER QUALITY AND SELF-CONTROL}

This thesis addressed whether ethanol, as a reinforcer, is qualitatively different from other appetitive reinforcers. Of particular interest was how reinforcer quality, which was altered by adding ethanol to typical appetitive reinforcers, interacted with delay to influence behaviour in self-control paradigms. Studies examining oral selfadministration of ethanol by non-human animals show that, similar to humans, animals exhibit a binge or bout pattern of drinking (Samson, 2000). This pattern of behaviour can be discussed in terms of impulsivity. That is, consumption of ethanol to the point of intoxication (binging) is impulsive behaviour; and moderating ethanol intake is self-controlled behaviour. A second issue examined in this thesis was whether existing self-control paradigms are sufficient analogues of human behaviour when examining impulsivity in the context of addiction or alcohol dependence. Two animal paradigms were used. The first was a concurrent chains procedure similar to those previously used to examine the mechanisms behind choice behaviour for alternatives that differ in terms of magnitude and/or delay (Davison, 1983; Grace, 1999; Rachlin \& Green, 1972). The second paradigm, used in Experiments 2 and 3, was a novel paradigm developed specifically for use in this thesis. The novel paradigm addressed self-control situations in which the choice was between two immediately available reinforcers and their associated delayed reinforcers (Peters, Hunt, \& Harper, 2004). Delays to reinforcer delivery and reinforcer quality were manipulated in both paradigms. The implications for theoretical models of choice behaviour are discussed.

Two existing models of choice behaviour, the Generalised Matching Law (GML, Baum, 1974) and the Contextual Choice Model (CCM, Grace, 1994), were originally 
developed to describe behaviour allocation between qualitatively identical reinforcer alternatives in concurrent and concurrent-chains schedules. Variations of the GML, have been used to describe data obtained from a limited number of studies that examine the impact of reinforcer quality on response distribution in concurrent schedules (e.g., Hollard \& Davison, 1971; Martinetti, Andrzejewski, Hineline, \& Lewis, 2000; Matthews \& Temple, 1979). The ability of the CCM to account for behaviour in situations in which the scheduled alternatives deliver qualitatively different reinforcers has not been assessed. This thesis examined whether these two models accurately described behaviour when concurrently available alternatives delivered qualitatively different reinforcers. The experiments included in this thesis examined reinforcers that were made qualitatively dissimilar by adding a physiologically active drug, ethanol, to commonly studied primary reinforcers. Existing studies of choice between drug-containing and drug-free reinforcers have equivocal results (Anderson, Velkey, \& Woolverton, 2002; Heyman \& Oldfather, 1992). Ethanol, in particular, appears to result in behaviour that deviates from that typically seen with non-drug reinforcers. One purpose of this thesis was to determine whether equations developed to describe behaviour reinforced by non-drug reinforcers would accurately model the deviations in behaviour found when ethanol was used as a reinforcer.

\section{Ethanol Consumption and Drug Abuse}

The goal of animal models of drug consumption is to mirror human drug abuse behaviour and thereby gain greater understanding of the mechanisms controlling drug consumption. Animal models are effective tools for investigating drug consumption because strict experimental controls are possible (Koob, 2000; Wolffgramm, Galli, Thimm, \& Heyne, 2000). A major component of ethanol dependence is the inability 
to regulate drug intake (DSM-IV). Animal models of ethanol dependence try to induce subjects to consume ethanol in ways that emulate the behaviour of human addicts (Samson, 2000). Studies using randomly bred rats have found that rats readily consume both unsweetened (Beardsley, Lemaire, \& Meisch, 1993; Czachowski \& Samson, 1999; Martinetti et al., 2000; Samson, Pfeffer, \& Tolliver, 1988) and sweetened (Czachowski \& Samson, 1999; Heyman, 1993; Heyman \& Oldfather, 1992; Samson et al., 1988) ethanol solutions in volumes sufficiently large to have physiological effects. Repeated consumption of ethanol by rats causes behaviours, similar to that of ethanol dependent humans, including sensitisation evidenced by increased locomotor activity prior to ethanol consumption (Wolffgramm et al., 2000), tolerance demonstrated by increased consumption (Wolffgramm et al., 2000) and withdrawal indicated by increased acoustic startle response (Macey, Schulteis, Heinrichs, \& Koob, 1995; van Erp \& Miczek, 2001) and by an increase in anxiety related behaviour (Rasmussen, Mitton, Green, \& Puchalski, 2001).

For many animal studies (again considering the behaviour of randomly bred rats), ethanol consumption patterns are described in terms of drinking bouts and inter-bout intervals (Samson, 2000). A drinking bout is a single episode of drinking and the size of a bout is measured by how much ethanol $(\mathrm{g} / \mathrm{kg})$ is consumed. An inter-bout interval is the time between drinking episodes (bouts). As tolerance develops, ethanol consumption increases and that increase may be in the form of shorter inter-bout intervals (i.e. more frequent bouts) or increases in bout size. Rats in a continuous access paradigm most frequently regulate their ethanol consumption through changes in inter-bout intervals (Samson, 2000). In continuous access paradigms, the amount of ethanol consumed in a drinking bout may decrease (if, for instance, the response requirement to obtain the ethanol increases) but it is unusual for bout size to increase 
(Samson, 2000). In contrast, ethanol consumption in limited access paradigms (i.e. all ethanol is consumed by the subject within a restricted time limit such as a 30 minute experimental session) tends to begin with an initial bout in favour of ethanol followed by a shift to consume more of the drug-free alternative as the session continues (Nowak, McKinzie, McBride, \& Murphy, 1999). When using randomly bred rats it is difficult to ensure that subjects consume enough ethanol to experience the physiological effects within a session, particularly if ethanol is diluted with plain water. However, some induction methods do result in consumption of relatively high concentrations of ethanol (up to $40 \%$, Samson, 1986; Samson et al., 1988). The most reliable method of increasing ethanol consumption in a given drinking bout is to deliver the ethanol in a sweetened solution (Samson, 2000). A benefit of adding sucrose (or another sweetening agent such as saccharin) to an ethanol solution is that both drug-naïve and drug-experienced animals will consume ethanol at concentrations greater than if the drug is available in water (Heyman \& Oldfather, 1992; Samson et al., 1988). A common argument against using sweetened solutions to investigate ethanol consumption is that it is difficult to determine whether the physiological effects of ethanol, the caloric value of ethanol, or the quality of the sweetener is controlling behaviour. However, evidence suggests that when rats respond for ethanol solutions it is to obtain the physiological drug effects (Files, Samson, \& Brice, 1995; Heyman, 1993; Heyman \& Oldfather, 1992; Samson, 1986). One method of addressing the argument regarding the use of sweetened solutions is to establish that ethanol is reinforcing for subjects prior to using an adulterated ethanol solution to reinforce operant responding. 
One purpose of this thesis was to determine if reinforcer quality impacted on response allocation when responding maintained by a drug-containing reinforcer reduced total reinforcement delivered in a trial. Two self-control paradigms were used to study this issue. Another aim was to examine whether two existing mathematical models of choice could quantitatively describe individual subject's behaviour distribution between qualitatively different reinforcers based on the data obtained in the operant paradigms.

\section{Quantifying Choice}

\section{Concurrent Schedules and the Generalised Matching Law}

The study of choice behaviour initially used concurrent schedules to determine how manipulating variables such as the type of schedule or the reinforcement rate influenced behaviour. A concurrent schedule paradigm typically uses two response operanda and subjects are able to distribute their responses between the two alternatives. Herrnstein $(1970 ; 1974)$ mathematically described the relationship between behaviour and reinforcement in concurrent schedules. The equation he developed, the strict matching law (Equation 1), states that an individual provided with two sources of reinforcement will allocate behaviour between the alternatives in proportion to the amount of reinforcement each alternative delivers.

$$
\frac{B_{1}}{B_{1}+B_{2}}=\frac{R_{1}}{R_{1}+R_{2}}
$$

In Equation 1, $B_{1}$ and $B_{2}$ are the amounts of behaviour allocated to each alternative and $R_{1}$ and $R_{2}$ are the rates of reinforcement delivered for responding on each alternative. Strict matching and subsequent developments in matching theory (see 
below) have been found to describe concurrent schedule response distribution in species as diverse as pigeons, rats, cows, and humans (Davison \& McCarthy, 1988).

Strict matching occurs in situations in which relative behaviour distribution is exactly equal to reinforcement distribution. As Herrnstein's equation was applied to increasingly complex choice situations systematic deviations from strict matching became evident. Baum $(1974 ; 1979)$ developed the GML to account for behaviour distribution that did not conform to strict matching (Equation 2).

$$
\frac{B_{1}}{B_{2}}=c\left(\frac{R_{1}}{R_{2}}\right)^{a}
$$

$B_{1}$ and $B_{2}$ are the responses distributed to alternatives 1 and $2, R_{1}$ and $R_{2}$ are the number of reinforcers available on each alternative, $c$ is inherent bias to one of the two alternatives and $a$ is sensitivity to relative reinforcement rate. The logarithmic form of the GML (Equation 3) proposed by Davison and McCarthy (1988) enables deviations from strict matching to be easily identified using linear regressions.

$$
\log \left(\frac{\mathrm{B}_{1}}{\mathrm{~B}_{2}}\right)=\mathrm{a} \cdot \log \left(\frac{\mathrm{R}_{1}}{\mathrm{R}_{2}}\right)+\log \mathrm{c}
$$

In Equation 3, the parameter $a$ is the slope of the line and indicates sensitivity to reinforcement distribution across alternatives. Sensitivity reflects how closely the subject's response allocation matches reinforcement allocation. If $a>1$ the subject has overmatched, that is, the distribution of the subject's responses across the alternatives is more extreme than the distribution of reinforcers would predict. If $a<$ 
1 , which is described as undermatching, the distribution of the subject's responses is less extreme than predicted by the reinforcement distribution. If $a=1$ behaviour distribution matches reinforcement distribution (Baum, 1974, 1979). Also from Equation 3, bias $(\log c)$ is the $y$-intercept of the line where $\log c>1$ indicates the subject is biased towards alternative 1 and $\log c<1$ shows bias to alternative 2 . If $\log$ $c=0$ there is no bias.

The extent to which undermatching occurs $(a<1)$ indicates how well the subject has discriminated between the actual rates of reinforcement delivered on each alternative. Strict matching occurs when there is perfect discrimination $(a=1)$. Reviews of concurrent choice studies using human and non-human animals have found that sensitivity to the reinforcement alternatives is typically less than one (Baum, 1979; Davison \& McCarthy, 1988; Robinson, 1992; Wearden \& Burgess, 1982). For example, in a review, Robinson (1992) found consistent undermatching and evidence that sensitivity ( $a$ values) varied by species (e.g. the mean $a$ value was 0.86 for pigeons and 0.92 for rats).

Sensitivity to the reinforcer ratio does not appear to be systematically influenced by changes in reinforcer quality (Anderson et al., 2002; Trevett, Davison, \& Williams, 1972). Although the sensitivity parameter does not capture the effect of quality manipulations on response distributions in concurrent access situations, the bias parameter $(\log c)$ may be able to. The bias parameter was initially used to account for non-experimentally controlled variables that systematically contributed to response distribution (Baum, 1974). For instance, if a pigeon had a preference for red over green it could develop a response bias and respond on red keys more frequently than 
green ones, regardless of the scheduled reinforcement contingencies. Studies devoted to examining the influence of rate, immediacy, and amount on response allocation in concurrent and concurrent chains schedules typically find that the bias parameter, log $c$, is close to 0 (Robinson, 1992). That is, experimenter control is usually strict enough that unbiased responding occurs when using qualitatively identical reinforcers.

There are situations in which bias does systematically vary and it may be that bias can be used as a measure of the effect of some independent variables such as quality. According to Baum (1974) possible variables causing bias include; " (3) qualitatively different reinforcers." (p. 234). Experimental manipulations of this variable should thus produce systematic changes in bias and there is empirical evidence supporting the use of this measure. As an example, Hollard and Davison (1971) manipulated bias by delivering different quality reinforcers (grain $v s$. ectostriatal brain stimulation). The authors found that response distributions produced biased matching and provided evidence that reinforcer quality influences preference. They found that the pigeons preferred food to ectostriatal brain stimulation and it is clear that their data could be described by Equation 3 in which the quality manipulation would result in a change in the $y$-intercept.

The GML presents one way of examining the influence of quality for reinforcers that differ in terms of rate and quality of reinforcement. However, this thesis focuses on the influence of quality in a self-control context in which reinforcers differ not in terms of rate but in terms of reinforcer magnitude and immediacy. A more 
sophisticated model of response allocation may be necessary to describe self-control choice and the effect of quality in this context.

\section{The Concatenated Matching Law}

Although early studies of concurrent schedule behaviour indicated that response allocation was a function of reinforcer rates (Catania, 1963; Fantino, 1969), research also indicates that the distribution of behaviour correlates with several other variables. These include reinforcer immediacy (Hursh \& Fantino, 1973; McDiarmid \& Rilling, 1965), and amount (or magnitude) of reinforcement (Fantino \& Herrnstein, 1968; Green, Myerson, Holt, Slevin, \& Estle, 2004; White \& Pipe, 1987), as well as probability of reinforcement (Logan, 1965), and reinforcer concentration (Farrar, Kieres, Hausknecht, de Wit, \& Richards, 2003; Martinetti et al., 2000). If the combination of variables associated with a reinforcement alternative constitutes the value of that alternative (Baum \& Rachlin, 1969; Mazur \& Kralik, 1990), then distribution of responses between alternatives may be proportional to the relative value of each alternative (Baum \& Rachlin, 1969; Rachlin, 1971).

The matching law can be adapted to account for the influence of multiple independent variables on preference. One extension of the matching law (Equation 4) states that the ratio of values of reinforcer alternatives is a multiplicative function of the ratios of the independent variables programmed for each schedule (Baum \& Rachlin, 1969; Rachlin, 1971).

$$
\frac{V_{1}}{V_{2}}=\left(\frac{R_{1}}{R_{2}}\right)\left(\frac{A_{1}}{A_{2}}\right)\left(\frac{D_{1}}{D_{2}}\right)\left(\frac{x_{1}}{x_{2}}\right)
$$


Equation 4 states that $V$ (the value of a reinforcer alternative as measured by the responding on that alternative) is equal to the multiplication of the independent variables that contribute to that alternative including: reinforcer rate (R), amount (A), and delay (D). The parameter $x$ represents any other variable that can be experimentally manipulated. Once again, the subscripts ${ }_{1}$ and ${ }_{2}$ are the two alternatives. In principle, the concatenated matching law could describe behaviour * found in studies of self-control behaviour. Such studies frequently examine response distribution between reinforcers of unequal amount and immediacy. A modified generalised matching equation (Equation 5) that incorporates the influence of reinforcer amount and delay is derived using the concatenated matching law (Logue, Rodriguez, Pena-Correal, \& Mauro, 1984).

$$
\frac{B_{1}}{B_{2}}=b\left(\frac{R_{1}}{R_{2}}\right)^{a_{R}}\left(\frac{A_{1}}{A_{2}}\right)^{a_{A}}\left(\frac{D_{2}}{D_{1}}\right)^{a_{D}}
$$

The advantage of the generalised concatenated matching law (GCML), Equation 5, is that the free parameters $a_{R}, a_{A}$ and $a_{D}$, which are sensitivity to rate, amount and delay, account for between subject preference differences (Grace, 1999) and make it possible to describe individual data obtained from self-control paradigms.

\section{Self-Control Paradigms}

Paradigms have been developed to extend studies of choice between concurrently available reinforcement schedules to the experimental analysis of self-control (Ainslie, 1974; Green, Fisher, Perlow, \& Sherman, 1981; Grosch \& Neuringer, 1981; 
Mazur \& Logue, 1978; Rachlin \& Green, 1972; Warry, Remington, \& Sonuga-Barke, 1999). In these studies, and others investigating self-control, both delay and amount differ between the alternatives. A response reinforced with a small amount of reinforcer after a brief delay is defined as impulsive; and a response reinforced with a larger amount of reinforcer after a longer delay is defined as self-controlled. A central concept in the study of self-control is preference reversal. Preference reversal occurs in a two alternative choice paradigm when behaviour shifts from one reinforcement alternative to the other (Green et al., 1981; Rachlin \& Green, 1972). In self-control contexts, the preference shift occurs between the impulsive and the self-controlled choice options.

According to the concatenated matching law, holding the difference between delays constant while increasing the absolute delay to delivery of both a smaller more immediate reinforcer (SI) and a larger more delayed reinforcer (LD) changes the behaviour ratio. As the absolute delay increases, the behaviour ratio should change from preference for the SI to preference for the LD due to the process of temporal discounting of reinforcer value and is dependent on the relative (rather than the absolute) delays to the two reinforcer alternatives. That is, as the time between when an individual must choose between the concurrently available reinforcer alternatives and actual delivery of the reinforcers increases, the value of the reinforcers decrease. Matching theory does not explain the mechanism behind reinforcer discounting, but Equation 5 predicts that, if the delay ratio remains constant and the absolute delays are increased, all else being equal, the relative value of the alternatives will remain constant and the behaviour ratio should not change. However, if the delay ratio 
changes as delay to reinforcer delivery increases, the behaviour ratio should change and preference should shift from one alternative to the other.

Rachlin and Green (1972) designed a paradigm to test whether changing the delay ratio to reinforcer delivery would result in a preference shift from a SI reinforcer to a LD reinforcer (Figure 1.1). In the 1972 Rachlin and Green study, pigeons were required to respond on concurrent-chain schedules. The initial link response requirement was a fixed ratio (FR) 25 and meeting the criterion gave the subject access to the terminal link. Primary reinforcement was delivered in the terminal links, which were separated from the initial links by an adjustable delay. One terminal link presented the subject with a choice between $2 \mathrm{~s}$ of food delivered immediately (the impulsive choice) and $4 \mathrm{~s}$ of food delivered after a $4 \mathrm{~s}$ delay (the self-controlled choice). The other terminal link was arranged so that only the self-controlled alternative was available. Thus, if a pigeon completed the FR schedule on the initial link key associated with the self-controlled alternative they were able to commit to the self-controlled reinforcer. Subjects that chose the terminal link in which they could decide between the reinforcement options a second time were inevitably impulsive.

Rachlin and Green predicted that, as the delay $(\mathrm{T})$ between the initial link and terminal reinforcement increased, the pigeons would reverse their preference from the impulsive to the self-controlled option. Three of the five pigeons demonstrated preference reversal. Such variability between subjects in terms of behaviour and preference reversal may result from individual differences in reinforcement history (Mazur \& Logue, 1978). One bird demonstrated preference for the key associated with commitment to the self-control alternative at all delays (and that preference 
increased as delay to the terminal reinforcer increased), the other was impulsive at all delays but was beginning to respond to the self-controlled option more frequently at the longer delays. Thus, the failure to find preference reversal in those subjects may have been due to the limited delays examined.

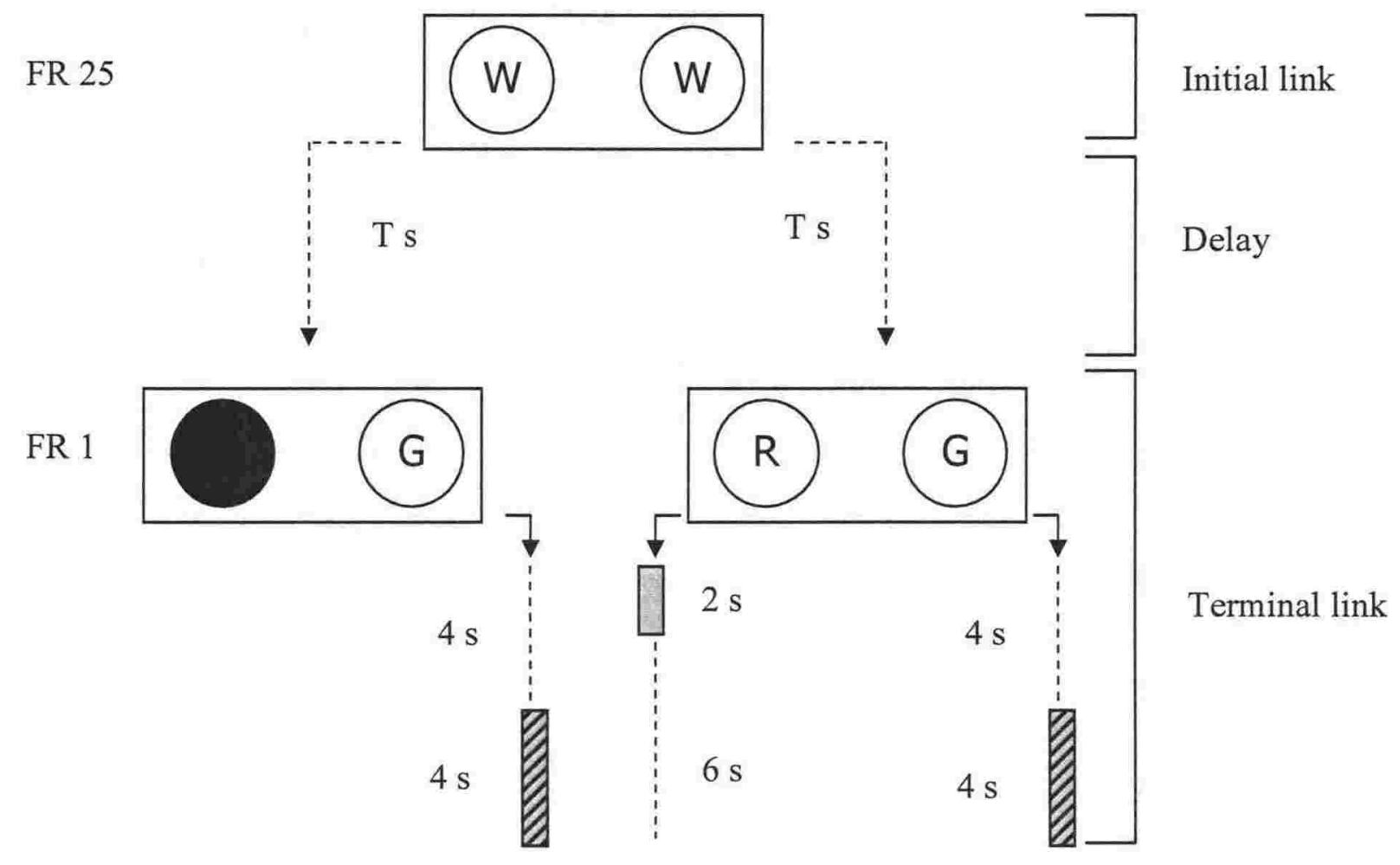

Figure 1.1. Concurrent-chains procedure adapted from Figure 1 Rachlin and Green (1972). The FR schedules on the left of the diagram indicate the response requirement for each link. Pigeons distributed their responses between the two keys (lit white, W) in the initial link. The key on which the last response was made determined which terminal link was entered. A final response on the left key blacked out one key, lit the second key green $(\mathrm{G})$, and committed the subject to the self-controlled terminal reinforcement contingency. A final response on the right key lit one key red (R) and other key green. The subject was then given a second opportunity to choose between the two reinforcement options. The shaded bars represent primary reinforcement delivery and the dashed lines represent delays. The delay $(\mathrm{T})$ between termination of the initial link and the reinforcement phase of the terminal link was the same for both of the alternatives

A simple concatenated matching law was unable to predict the behaviour of the pigeons in the Rachlin and Green study. However, variations of this procedure in which individuals choose between SI and LD reinforcers, have been used extensively to experimentally analyse the processes underlying self-control behaviour in human 
(Dziadosz \& Tustin, 1982; Green et al., 2004; Green, Myerson, \& Ostaszewski, 1999; Kirby \& Herrnstein, 1995), and non-human animals (Green et al., 1981; Ito \& Asaki, 1982; Logue \& Pena-Correal, 1984; Mazur, 1988; Reynolds, de Wit, \& Richards, 2002).

Trial duration varied in Rachlin and Green (1972) as time to reinforcement delivery (T) varied. Green et al. (1981) suggested that the failure to find consistent preference reversal using the commitment procedure might have been because subjects' response latencies decreased scheduled reinforcer immediacies. Thus, Green et al. (1981) modified the concurrent chains procedure to control for trial duration. All trials in the modified procedure lasted $30 \mathrm{~s}$ and delivery of the reinforcer contingencies commenced $10 \mathrm{~s}$ before the end of each trial. As the delay between choice and reinforcer delivery increased, all four pigeons in Green et al.'s study reversed preference from the SI reinforcer to the LD reinforcer. Thus, by controlling for variations in reinforcer immediacy Green et al. successfully demonstrated preference reversal in pigeons. Preference reversal was also shown in mentally retarded children working on tasks associated with either SI or LD reinforcers (Dziadosz \& Tustin, 1982). Children chose which tasks they would complete. In the free choice conditions the choices were made within sessions. In the commitment conditions, tasks were chosen 24 hours in advance of the daily sessions. Reinforcement for completing the SI-associated tasks was immediate and the LD-associated tasks were reinforced 30 minutes after completion. The children tended to be impulsive and chose to work on SI tasks in the free choice conditions, but self-control increased during commitment conditions. This application of a paradigm developed from animal research demonstrates the usefulness of basic experimental analysis for identifying techniques to increase self-control in humans (Dziadosz \& Tustin, 1982). 
A different procedure was used by Kirby and Herrnstein (1995) with high school and undergraduate students who chose between monetary reinforcers in two experiments; and between items of merchandise (such as a Timex watch or a Swiss army knife) in a third study. In their third study, Kirby and Herrnstein (1995) examined the effect of qualitatively different reinforcers on preference in a two alternative choice paradigm. This study used a titration procedure rather than a concurrent chains procedure. In titration procedures, a subject indicates their preference for one of two reinforcer alternatives. One alternative is available after a short delay (SI) and the other is available after a longer delay (LD). The delay to one of the reinforcers is adjusted after each trial. For example, on the first trial a subject presented with a choice between receiving $\$ 10$ immediately or $\$ 100$ in one week may say they prefer to receive the $\$ 100$. On the following trial, the delay to the $\$ 100$ is increased to one month and the subject is again asked which monetary alternative they would prefer to receive. This time they may say they would prefer the $\$ 10$. The delay continues to be adjusted on each trial depending on which alternative the subject said they preferred on the previous trial. The point of the titration procedure is to determine at which pair of delay and amount ratios the subject is indifferent between the two alternatives. Once a series of indifference points have been established it is possible to determine the rate at which delay discounts reinforcer value. The students in Kirby and Herrnstein's study all reversed their preferences from the SI to the LD reinforcer alternatives as delay to reinforcer delivery increased thus demonstrating that existing self-control paradigms can be used to examine choice between qualitatively dissimilar reinforcers. 
Hyperbolic discounting of delayed reinforcers predicts the preference reversals found by Kirby and Herrnstein (1995) and Dziadosz and Tustin (1982). Consider Figure 1.2 (adapted from Green et al. 1981, Figure 1), in which two reinforcer alternatives of different magnitudes and immediacy are available. The taller hatched bar and its accompanying curve is the self-controlled alternative and the shorter solid bar and associated function is the impulsive alternative. The point on the discount functions when the choice is made reflects the relative value of each reinforcer. If the choice is made at $\mathrm{t} 1$, when there is a reasonably large delay before reinforcer delivery is scheduled, the function for the larger reinforcer is above that of the smaller reinforcer and the self-controlled option would be chosen. As time to reinforcer delivery decreases the value of each reinforcer increases. At $t 2$ the curves cross and the alternatives are equally valuable and by $\mathrm{t} 3$ the discount function for the impulsive option is now above that of the self-controlled choice indicating that if the choice response was made at that point in time it would favour the impulsive alternative.

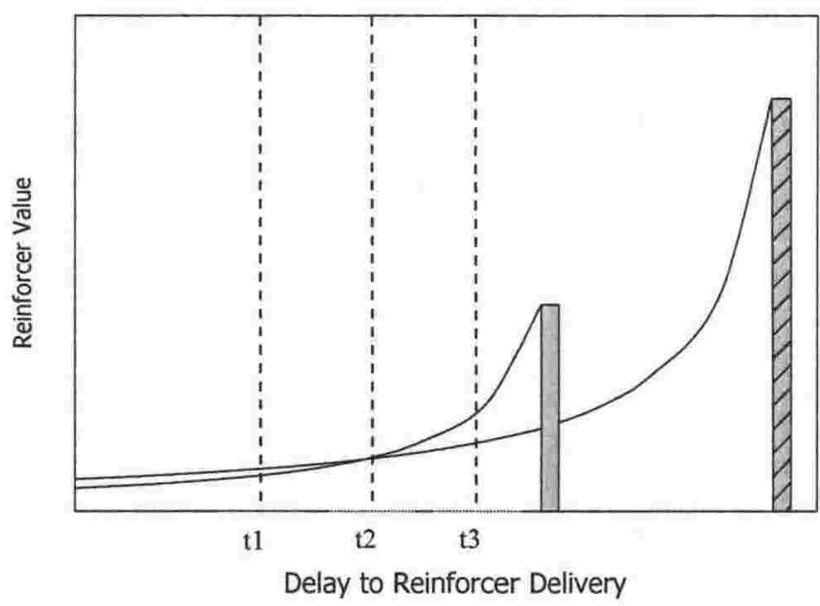

Figure 1.2. Diagram of the hyperbolic discounting functions for two unequal magnitude reinforcers. The two curves indicate the temporally discounted values of the reinforcers. Reinforcer value increases along the $y$-axis and delay to reinforcer delivery decreases from left to right along the $\mathrm{x}$-axis. The plain bar represents the impulsive alternative and the hatched bar represents the self-controlled alternative. 
Before alternative models of choice behaviour are discussed, the impact of quality of reinforcement on response allocation between concurrently available reinforcers are considered (Chelonis \& Logue, 1997; King \& Logue, 1990; Kirby \& Herrnstein, 1995; Miller, 1976). One way of accounting for the impact of reinforcer quality on response distribution in concurrent chains is to regard quality as an independent variable that functions similarly to other independent variables such as immediacy and amount of reinforcement. If this is true then quality may be added to Equation 4 (as shown in Equation 6). The parameters in Equation 6 are identical to Equation 4 except that the variable $Q$ is a constant representing reinforcer quality.

$$
\frac{V_{1}}{V_{2}}=\frac{R_{1}}{R_{2}} \cdot \frac{A_{1}}{A_{2}} \cdot \frac{I_{1}}{I_{2}} \cdot \frac{Q_{1}}{Q_{2}}
$$

It is difficult, however, to determine how a qualitative variable should be quantified. If the qualities are equal, as in most studies of concurrent schedules, then the quality ratio will equal 1 and should not affect response distribution. If qualities are unequal then the problem remains of how to quantify the difference between the two reinforcer alternatives.

There is some evidence in the literature that reinforcer quality can be accounted for by Equation 6. Miller (1976) examined response allocation between concurrent VI VI reinforcer alternatives that delivered reinforcers of substitutable quality (hemp, buckwheat, and wheat). The matching equation was modified and applied to the results (Equation 7). Equation 7 is a logarithmic transformation of Equation 6. 


$$
\log \left(\frac{V_{1}}{V_{2}}\right)=a \cdot \log \left(\frac{R_{1}}{R_{2}}\right)+\log \left(\frac{Q_{1}}{Q_{2}}\right)
$$

For Equation $7 V, R$, and $Q$ are: reinforcer value (measured as the behaviour ratio $\left.\mathrm{B}_{1} / \mathrm{B}_{2}\right)$, rate, and quality and the subscripts indicate the reinforcer alternatives. Miller (1976) first determined preference between two pairs of grains and then used those values to predict the preference ratio of a third pair. Miller found that subjects divided their responses relative to the reinforcer rates and were biased towards the alternative delivering the more preferred grain. Predictions for the preference ratio for the third pair of grains were most accurate when the slope $(a)$ was set equal to 1.0 but accuracy was still high when the derived value of $a$ (from the initial comparisons) was used. Thus, a concatenated matching equation can both describe and potentially predict the impact of quality manipulations using concurrent VI VI schedules. A similar method was used by (Sumpter, Foster, \& Temple, 1993) to predict whether hens responding for wheat would prefer responding on an alternative in which reinforcement delivery depended on five key pecks or on one that required a door push.

The ability of matching-law equations to account for the influence of manipulating reinforcer quality on preference may depend on whether substitutable or nonsubstitutable reinforcer qualities are used. Reinforcers of substitutable quality serve similar functions for the individual consuming them and unsubstitutable reinforcers have different functions (Green \& Fisher, 2000). Manipulating reinforcer quality by adding ethanol to a sucrose solution changes behaviour in concurrent access situations. Heyman and Oldfather (1992) found that when sucrose was delivered on 
concurrent VI VI schedules, increasing the requirement for one of the schedules increased responding on the unchanged schedule. However, when one of the reinforcers was adulterated with ethanol increasing the response requirement for the alternative that was reinforced with the drug-containing reinforcer did not decrease responding for that alternative. That is, response distribution when qualitatively unequal reinforcers were concurrently available was dissimilar to response distribution between qualitatively equal reinforcers. (Hursh, 1980) suggested that the relative reinforcing efficacy of a reinforcer such as ethanol depends on what other reinforcers are concurrently available. That is, if the concurrently available reinforcers were qualitatively identical then the alternatives are substitutable and changing the reinforcement schedule associated with one alternative should simply switch responding to the other alternative. However, if the alternatives are not substitutable response allocation is less likely to shift from the changed to the unchanged alternative (e.g., Heyman and Oldfather, 1992). This has implications for models of choice behaviour. In cases where one of the alternatives delivers a pharmacologically active reinforcer (such as ethanol) it may be that variables that increase or decrease responding maintained by that reinforcer differ from those that influence responding for a concurrently available reinforcer.

Few studies have examined the influence of reinforcer quality on the phenomenon of preference reversal in rats. Some findings suggest that reinforcer quality influences choice behaviour by changing sensitivity to the independent variables such as sensitivity to delay and amount. Chelonis and Logue (1997) compared self-control for water in water-deprived rats to self-control for food in food-deprived rats. They found that behaviour reinforced with water was more self-controlled than behaviour 
reinforced with food. The GML was applied to the results and Chelonis and Logue determined that sensitivity to delay was greater in the food-deprived rats as compared to the water-deprived rats. In a similar study, Green and Estle (2003) employed a within-subject self-control paradigm with rats and found that preference reversal occurred for both food and water reinforcers. For that study, the delay at which subjects were impulsive and chose the SI over the LD was individually determined for each subject. Next, a constant delay was added to the delivery of the SI and the LD reinforcers, the subjects all increased responding for the LD as the delay to the reinforcers increased. That is, as delay to both of the reinforcers increased preference shifted from favouring the impulsive alternative to favouring the self-controlled alternative. These two studies demonstrate that concurrent chains choice procedures are a useful approach for examining the influence of reinforcer quality on choice. However, both of these studies studied one quality of reinforcer at a time.

Behavioural allocation was examined when both alternatives delivered food or when both alternatives delivered water and then behaviour from the two conditions was compared. A comparison of choice between qualitatively different reinforcers delivered in the same condition (i.e. when one alternative delivered water and the other alternative delivered food) was not included in either of these studies. The first experiment of this thesis initially examined the effect of manipulating reinforcer quality on choice proportions when both of the reinforcer qualities were identical and then compared the results with results from conditions in which different quality reinforcers were delivered for the SI and the LD alternatives of a self-control paradigm. 


\section{The Contextual Choice Model}

In 1994, Grace developed the CCM, which is an extension of the GML, to account for the effect of temporal context on response allocation in concurrent chain schedules. In particular, Grace's model addressed the occurrence of both the initial link effect and the terminal link effect. The initial link effect is that as the initial links of a concurrent chains procedure increase in duration sensitivity to the initial link contingency increases. The terminal link effect occurs when sensitivity to the terminal link contingencies decreases as the duration of the initial links increases. Thus, as noted by Grace (1994), there appears to be an interaction between sensitivity to the terminal links and initial link duration for concurrent-chains procedures. Grace (1994, 1999) proposed Equation 8 as a method of accounting for temporal context effects using a model developed from the generalised concatenated matching law.

$$
\frac{B_{1}}{B_{2}}=b\left(\frac{R_{1}}{R_{2}}\right)^{a_{R}}\left[\left(\frac{D_{2}}{D_{1}}\right)^{a_{D}}\left(\frac{x_{1}}{x_{2}}\right)^{a_{X}}\right]^{\left(\frac{T_{t}}{T_{i}}\right)^{k}}
$$

The parameters of the CCM are as follows; $\mathrm{R}$ is initial link reinforcement rate, $\mathrm{D}$ is delay to terminal link primary reinforcement, $\mathrm{x}$ represents another independent variable (such as reinforcer amount), and $k$ is a scaling parameter. The subscripts 1 and ${ }_{2}$ refer to the two concurrent schedules. The sensitivity parameters are sensitivity to the initial link reinforcement rate $\left(a_{R}\right)$, sensitivity to the terminal link delay $\left(a_{D}\right)$ and sensitivity to independent variable $\mathrm{x}\left(\mathrm{ax}_{\mathrm{x}}\right)$. The parameters that determine the influence of temporal context are $T_{t}$ and $T_{i}$, which are the average terminal and initial link durations. As the duration of the terminal link $\left(\mathrm{T}_{\mathrm{t}}\right)$ increases, the contextual exponent increases in value and hence the influence of terminal link contingencies on initial link behaviour distribution increases. Likewise, increasing the duration of the 
initial link $\left(\mathrm{T}_{\mathrm{i}}\right)$ decreases the value of the contextual exponent and decreases the effect of the terminal link on initial link response allocation. Essentially, CCM assumes that the more time a subject spends in the presence of terminal link stimuli, the greater the influence the terminal link contingencies will have on initial link response distribution. "CCM is based on the assumption that the ratio of the average terminallink to initial link durations, $T_{t} / T_{i}$, is the crucial contextual variable in concurrent chains." (p. 126, Grace, 1994). Grace (1994) successfully applied CCM to 92 archival data sets. It has been demonstrated that CCM accounts for much of the variance in concurrent chains schedules in which the terminal links deliver equal magnitude reinforcers (Grace, 1994) or unequal magnitude reinforcers delivered after variable delays (Grace, 1995, 1999). However, the ability of the model to account for choices between qualitatively different reinforcers delivered after fixed delays has not been tested. Since much of the literature investigating self-control uses concurrent chains paradigms to examine choice between reinforcers of different magnitudes and fixed delays, it is a useful exercise to assess whether CCM can account for behaviour in self-control paradigms in which the reinforcers differ in terms of fixed (rather than variable) delay, magnitude, and quality. This issue is addressed in Experiment 1 and Experiment 3.

In summary, research into self-control has typically investigated choice between qualitatively identical reinforcers that are of unequal magnitude. Mathematical models that extend the Matching Law, which was originally developed to account for behaviour allocation between concurrent VI VI schedules, can describe behavioural allocation in such studies. What has not been addressed previously is whether choice behaviour adheres to the principles of matching theory when pharmacologically active 
reinforcers are used instead of appetitive or water reinforcers. The previous widespread use of ethanol in the behavioural pharmacology literature made it a logical choice to use in experiments that examine the impact of reinforcer quality on choice behaviour. The first experiment of this thesis examined the effect manipulating reinforcer quality had on choice proportions in an animal self-control analogue. The analogue used was a concurrent-chains paradigm and reinforcer quality was manipulated by adding ethanol to, or removing ethanol from, a sucrose solution. The second experiment introduced a novel self-control paradigm and investigated whether acclimatisation to ethanol consumption in subjects' home cages influenced operant responding for ethanol. The self-control procedure used in Experiment 2 was modified to reduce intrinsic side biases; and, the modified procedure was used in Experiment 3 to investigate further delay ratios and a different reinforcer vehicle. The reinforcer vehicle used in Experiment 2 was diluted sweetened condensed milk and the reinforcer vehicle used for Experiment 3 was sucrose water. Finally, in Experiment 4, the rats that served as subjects in the third experiment were studied using concurrent VI VI schedules to determine whether they preferred the ethanolcontaining reinforcer or the ethanol-free reinforcer used in Experiment 3. The overall hypothesis was that adding ethanol to food reinforcers would change response distribution in operant choice paradigms; and that the effect of reinforcer quality could be described by quantitative models derived from the Matching Law. 


\section{EXPERIMENT 1}

\section{Reinforcer Quality and Response Distribution in a Concurrent Chains Procedure}

Central to this thesis was the question of whether animals reinforced with ethanolcontaining reinforcers distribute their responses in a concurrent-chains paradigm differently than animals reinforced with drug-free reinforcers. Previous studies of behaviour allocation indicate that the introduction of drug-containing reinforcers may alter some behavioural phenomena that are evident when identical quality reinforcers are used (e.g., Heyman \& Oldfather, 1992).

Initial studies examining choice using traditional experimental paradigms that included ethanol as a reinforcer alternative did report differences relative to studies that did not use ethanol. For example, McSweeney, Melville, and Higa (1988) examined the influence of ethanol on behavioural contrast using concurrent and multiple schedules. Behavioural contrast is studied using two reinforcement schedules. Multiple schedules are typically used but concurrent schedules are also studied. The contingencies associated with one option are held constant while the contingences for the alternate behaviour are manipulated (for an example see Ettinger, McSweeney, \& Norman, 1981). Positive behavioural contrast is an increase in responding on the constant reinforcer alternative that occurs when the value of the alternate reinforcer option decreases. Negative behavioural contrast occurs when the value of the alternate reinforcer option is increased and responding on the constant alternative decreases. Using identical non-drug reinforcer qualities and varying amount of reinforcer for one alternative, Ettinger et al. (1981) found their results were 
well described by modifying the matching law so that relative rates of responding were set equal to relative amounts of reinforcement (Equation 9).

$$
\frac{B_{1}}{B_{1}+B_{2}}=\frac{A_{1}}{A_{1}+A_{2}}
$$

Ettinger et al. (1981) found positive and negative contrast when reinforcement amount was manipulated and both reinforcer options delivered mixed grain. Furthermore, when applying Equation 7, Ettinger et al. found bias and undermatching which is consistent with previous literature using concurrent schedules (e.g., Baum, 1974; Robinson, 1992; Wearden \& Burgess, 1982). Numerous other studies that have used qualitatively equal reinforcers and varied rate or amount of reinforcer in one component have produced similar findings (McSweeney, 1978; McSweeney \& Melville, 1993). In comparison, McSweeney et al. found that, when one alternative was ethanol, contrast was observed in concurrent schedules but not for multiple schedules. Likewise, Czachowski, Samson, and Denning (1999) found no evidence of behavioural contrast when ethanol and sucrose were available on multiple schedules. In related findings, Heyman (1993) and Heyman and Oldfather (1992) found that, within a behavioural economic framework, elasticity for an ethanol based reinforcer was not influenced by the availability of an alternate food reinforcer. That is, the extent to which demand for the reinforcer changed as a function of the increases in response requirement (elasticity) did not change when food was concurrently available. Similarly, Files et al. (1995) found that concurrent access to ethanolcontaining and ethanol-free reinforcers resulted in a stable $\mathrm{g} / \mathrm{kg}$ intake of ethanol (inelastic demand) across a variety of schedule/reinforcer pairings. In contrast, when 
reinforcers of the same quality are concurrently available elasticity typically increases (Hursh, 1980, 1991).

These differences found when drug rather than non-drug reinforcers are used may be explained with reference to a more general phenomenon rather than a drug effect per se. For instance, studies of behavioural contrast indicate that how a quality manipulation effects response distribution may depend on whether the reinforcers used are substitutable or not. Only under specific procedural conditions did Higa \& McSweeney (1987) find behavioural contrast with non-substitutable reinforcers (water and food) delivered on multiple schedules. No behavioural contrast occurred when the constant alternative was food and the variable alternative changed from food to water. However, changing the variable reinforcer from water to food led to decreased responding on the constant alternative (negative contrast) when the constant alternative was reinforced by water; but only when the rate of reinforcement was high (VI $15 \mathrm{~s}$ ). These studies into behavioural contrast and reinforcer quality suggest that manipulating reinforcer value by changing reinforcer quality may not necessarily influence behaviour in the same way as changing reinforcer amount or delay.

It remains unclear whether the models used to describe behaviour that is reinforced with food or water reinforcers can also describe choice behaviour when both alternatives provide the same quality reinforcers but the reinforcers are drugs. For instance, in a study by Martinetti et al. (2000) when different concentrations of ethanol were concurrently available in subjects' home cages the behaviour of only 16 of 28 rats was well described by a generalised matching equation when reinforcer concentration was assumed as analogous to reinforcer amount. Likewise, when 
rhesus monkeys chose between qualitatively different cocaine and food reinforcers in a simple concurrent VI VI the generalised matching law only described the data of 10 of the 16 subjects (Anderson et al., 2002). In contrast, using different cocaine doses, Anderson and Woolverton (2003) found that response distribution between reinforcer alternatives in a self-control paradigm was similar to behaviour in paradigms in which food was used as a reinforcer (Green \& Snyderman, 1980; Snyderman, 1983;

Williams \& Fantino, 1978). That is, as absolute, but not relative, delay to terminal reinforcer delivery increased preference shifted from the self-controlled (a higher dose after a longer delay) to the impulsive alternative (a lower dose after a shorter delay). Anderson and Woolverton's (2003) finding that substitutable drug reinforcers and substitutable food reinforcers had similar effects on behaviour suggests that it may be possible to investigate the impact of reinforcer quality on choice behaviour using a concurrent chains self-control paradigm. A previous study using a concurrent chains self-control paradigm to determine the impact of reinforcer quality on the parameters of matching based equations found that sensitivity to reinforcer amount and reinforcer delay were influenced by changes in reinforcer quality (Chelonis \& Logue, 1997). Thus, the impact of reinforcer quality may be understood within existing models if the use of qualitatively different reinforcers changes sensitivity to either the amount or delay. The behaviour of animals that are more sensitive to reinforcer delay should be more impulsive than that of animals that are more sensitive to reinforcer amount (Chelonis and Logue, 1997). Their results suggest that sensitivity to reinforcer delay and amount in the current study would depend on the quality of reinforcers used. However, the effect of ethanol reinforcers on behaviour in concurrent chains procedures has not been addressed to date. 
Therefore, the aim of this experiment was to determine if ethanol influenced the relative control exerted by delay and amount on behaviour using a self-control paradigm. Within the context of a self-control paradigm Green and Estle (2003) found that reinforcer quality changed the indifference points between concurrently available SI and LD reinforcers. In their study, indifference points for food reinforcers tended to occur at a shorter delay than the indifference points for water reinforcers which suggests that different quality reinforcers may be discounted at different rates. The first phase of the current experiment investigated whether there was any indication that the use of ethanol as a reinforcer would influence the typical pattern of behaviour on concurrent chain schedules in which the reinforcers differed in terms of delay and amount. Two groups of subjects were studied. The first group of subjects (the Ethanol Rats) had prior experience with ethanol-containing sucrose and were reinforced with ethanol-containing sucrose in the first phase of the experiment. The other group of subjects (the Plain Rats) did not have prior experience consuming ethanol and were reinforced with ethanol-free sucrose in the first phase. The second phase of the current experiment examined the effect, on selfcontrol behaviour, of using a drug-reinforcer as the SI and a non-drug reinforcer as the LD. If prior experience consuming ethanol caused the Ethanol Rats to become addicted to ethanol it was predicted that they would be more impulsive than the Plain Rats in Phase II. This was because the subjects would only be able to consume ethanol if they chose the SI reinforcer. Across both phases, the study investigated whether the effect of the reinforcer quality manipulations could be captured using a generalised version of the concatenated matching law. Based on Chelonis and Logue's (1997) suggestion it was predicted that increases in impulsivity would be reflected in an increase in sensitivity to delay relative to sensitivity to amount and 
increases in self-control would be reflected as an increase in sensitivity to amount relative to sensitivity to delay.

The choice paradigm used was a discrete trial concurrent-chains procedure. Rats were used as subjects. During the initial link of each trial, the subjects could respond on either the left or the right lever according to concurrent fixed interval (FI) $10 \mathrm{~s}$ FI $10 \mathrm{~s}$ schedules. Fixed interval schedules were used to ensure all animals spent the same amount of time in the initial link. The first lever on which the subject responded after the FI had elapsed determined terminal link entry. Responding on the lever associated with the impulsive choice was reinforced with one dip of reinforcer; and responding on the lever associated with the self-controlled choice was reinforced with three dips of reinforcer. There was a brief delay before the smaller reinforcer and a longer delay before the larger one.

Subjects were divided into two groups. During the first phase of the study, the Ethanol Rats were reinforced with a $10 \%$ ethanol sucrose solution (ethanol sucrose) for both the impulsive and the self-controlled choices; and the Plain Rats were always reinforced with an ethanol-free sucrose solution (plain sucrose). The lever with which each contingency was associated alternated across conditions. For the second phase, all subjects were reinforced with ethanol sucrose for each SI choice, and LD choices were reinforced with plain sucrose. Ethanol sucrose was used to reinforcer impulsive choices in Phase II both because consuming ethanol is often considered to be impulsive in non-experimental situations and due to time constraints. The experiment took approximately one year to complete and the subjects were a year old when the study began; the life expectancy for rats is approximately two years. It was predicted 
that any changes in individual subject's behaviour allocation in the second phase would be reflected as a change in the sensitivity ratio $\left(a_{A} / a_{D}\right)$ in Phase II as compared to the sensitivity ratio found in Phase I. Again, if the Ethanol Rats preferred ethanol they should be more impulsive than the Plain Rats and the difference between the groups should be reflected in a smaller sensitivity ratios for the Ethanol Rats.

Method

\section{$\underline{\text { Subjects }}$}

The subjects were six female Norway-hooded rats maintained at $85 \%$ of their freefeeding body weight by post-session feeding. A small sample was used because this was a pilot study designed to determine if the mathematical models developed to describe behaviour when a single reinforcer quality was used could also account for behaviour in a paradigm where more than one reinforcer quality was used. Water was freely available in the home cage. The rats were individually housed in cages $(42 \mathrm{~cm}$ x $20 \mathrm{~cm} \times 25 \mathrm{~cm}$ ) with opaque plastic bottoms and wire mesh tops. The housing room was on a 12-hour light/dark reverse cycle and lights came on at 7:30 pm. Testing was conducted during the dark cycle. Subjects were assigned to one of two reinforcer quality groups. Three rats were in the plain sucrose group (the Plain Rats) and three rats were in the ethanol sucrose group (the Ethanol Rats).

\section{Apparatus}

Six standard $30 \mathrm{~cm} \times 26 \mathrm{~cm} \times 34 \mathrm{~cm}$ operant chambers were used (Figure 2.1). Each chamber had two retractable levers, one fixed lever, four lights, and two dippers. The retractable levers were located on the front wall of the chamber $18.5 \mathrm{~cm}$ from the floor and $16.5 \mathrm{~cm}$ apart. The single fixed lever was centred on the back wall $6.5 \mathrm{~cm}$ above the chamber floor. Red lights were located $3.5 \mathrm{~cm}$ above each front lever, and $5 \mathrm{~cm}$ above the rear lever. A liquid dipper was located $12 \mathrm{~cm}$ below each retractable 
lever. Four of the chambers had dippers that had additional white lights that turned on when the dippers operated (Coulbourne Instruments H14-05R). Those dippers held $0.05 \mathrm{ml}$ of reinforcer. The other two chambers had dippers without white lights and delivered $0.04 \mathrm{ml}$ (MedAssociates ENV 202M) of reinforcer. There was a houselight in the centre of the operant chamber ceiling.

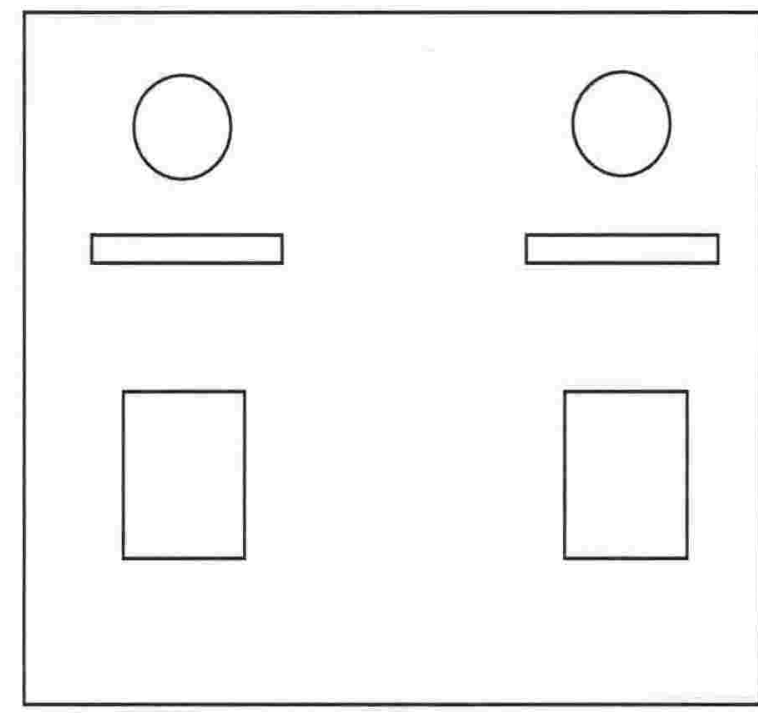

Lights

Retractable Levers

Dipper Wells

Figure 2.1. Schematic diagram of the front wall of the operant chamber showing the configuration of the lights, retractable levers, and dipper wells. The dipper located directly below the response lever delivered the reinforcer associated with a particular response.

\section{$\underline{\text { Reinforcers }}$}

The two reinforcers were plain sucrose and ethanol sucrose solutions. Fresh solutions were prepared daily. Plain sucrose was prepared by dissolving caster sugar in tap water $(\mathrm{w} / \mathrm{v})$ to obtain a solution of $10 \%$ sucrose concentration. The ethanol sucrose solution was prepared by adding enough ethanol (Ethanol absolute $\mathrm{C}_{2} \mathrm{H}_{5} \mathrm{OH}, 99.5 \%$ $\mathrm{v} / \mathrm{v}$ ) to the plain sucrose solution to make a reinforcer with a concentration of $10 \%$ ethanol $(\mathrm{v} / \mathrm{v})$. There were two phases for this experiment. During Phase I, the Plain Rats (F12, F32, and F52) were only reinforced with plain sucrose; and the Ethanol Rats (F22, F42, and F62) were only reinforced with ethanol sucrose. In Phase II, all six subjects were reinforced with ethanol sucrose for responding to receive the 
smaller, more immediate, terminal reinforcer and with plain sucrose for responding to receive the larger, more delayed terminal reinforcer.

\section{Experimental Contingencies}

Concurrent FI 10-s FI 10-s schedules operated in the initial links of all conditions

(Figure 2.2). The choice was always between a smaller, more immediate, reinforcer

(1 dip of reinforcer) and a larger, more delayed, reinforcer (3 dips of reinforcer).

Reinforcer magnitude (number of dips of reinforcer) was not varied in this

experiment. There was no delay between the initial and terminal links.

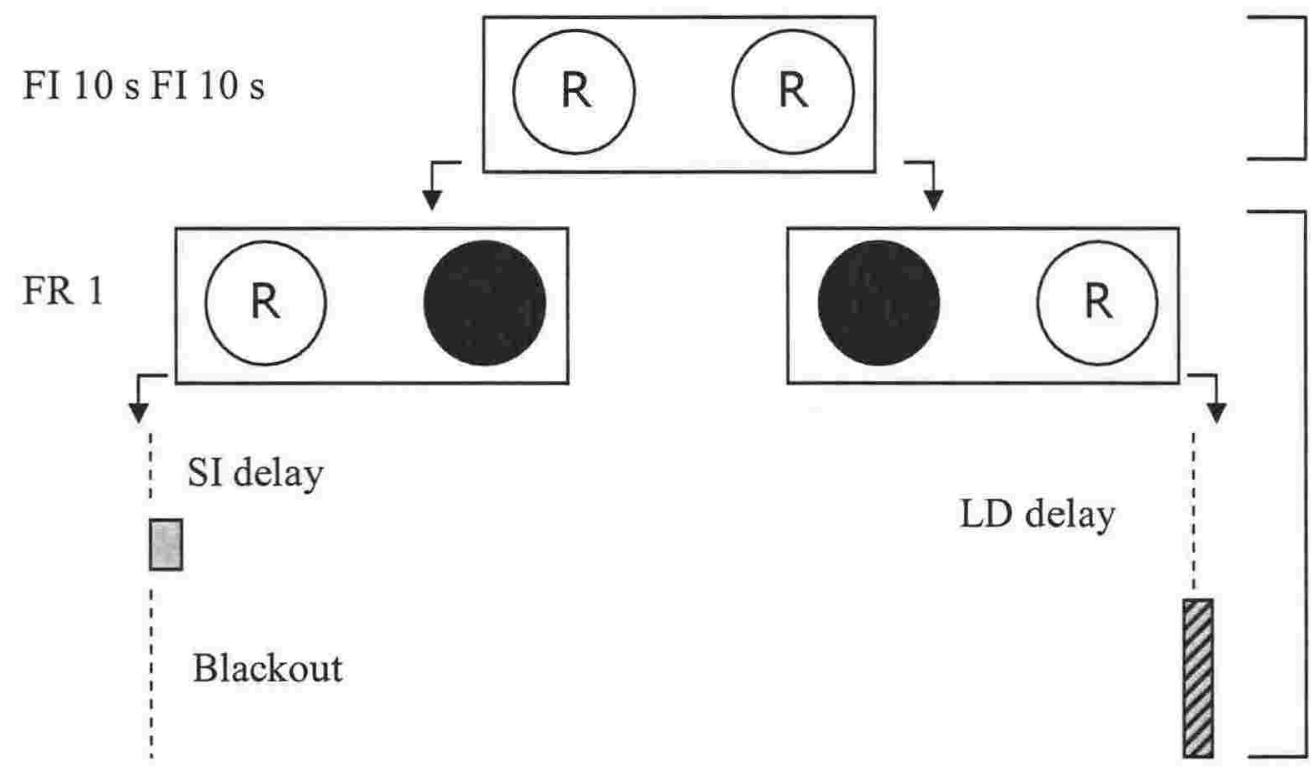

Initial link

Terminal link

Figure 2.2. Diagram of the concurrent chains self-control paradigm used in Experiment 1. Subjects respond in the initial link and are reinforced in the terminal link. The dashed lines indicate delay. Note that there was no delay between the initial and the terminal links. For the left impulsive alternative the light turned off after reinforcer delivery. The delay that followed delivery of the SI reinforcer ensured trial durations were equal. The solid bar represents delivery of the SI reinforcer (1 dip of reinforcer). The hatched bar indicates delivery of the LD reinforcer (3 dips of reinforcer). The location of the SI and LD reinforcers alternated across conditions.

The terminal links began with a delay to reinforcer delivery for both the SI and the

LD alternatives. The delay ratios varied across conditions and average time to

terminal reinforcer delivery was always $20 \mathrm{~s}$. The six delay ratios (SI:LD) were 1:2 
$\underline{\text { Table } 1.1}$

Condition order and terminal link delays to reinforcer delivery for Phase I and Phase II.

The Ethanol Rats were F12, F32, and F52 and the Plain Rats were F22, F42, and F62.

\begin{tabular}{|c|c|c|c|c|c|c|c|c|c|}
\hline \multirow[b]{2}{*}{ Sub } & \multirow[b]{2}{*}{ Cond } & \multicolumn{2}{|c|}{ Phase I } & \multicolumn{2}{|c|}{ Scheduled Delays } & \multicolumn{2}{|c|}{ Phase II } & \multicolumn{2}{|c|}{ Scheduled Delays } \\
\hline & & \multicolumn{2}{|c|}{ Delay Ratio } & \multirow{2}{*}{$\begin{array}{l}\text { Left } \\
5.71\end{array}$} & \multirow{2}{*}{$\begin{array}{l}\text { Right } \\
34.29\end{array}$} & \multicolumn{2}{|c|}{ Delay Ratio } & \multirow{2}{*}{$\begin{array}{l}\text { Left } \\
8.00\end{array}$} & \multirow{2}{*}{$\begin{array}{l}\text { Right } \\
32.00\end{array}$} \\
\hline F12 & 1 & 1 to 6 & (19) & & & 4 to 1 & (14) & & \\
\hline & 2 & 6 to 1 & (14) & 34.29 & 5.71 & 1 to 4 & (16) & 32.00 & 8.00 \\
\hline & 3 & 2 to 1 & (14) & 26.66 & 13.33 & 1 to 2 & (10) & 13.33 & 26.66 \\
\hline & 4 & 1 to 2 & (17) & 13.33 & 26.66 & 2 to 1 & (14) & 26.66 & 13.33 \\
\hline & 5 & 1 to 4 & (14) & 8.00 & 32.00 & 6 to 1 & (11) & 34.29 & 5.71 \\
\hline & 6 & 4 to 1 & (26) & 32.00 & 8.00 & 1 to 6 & (10) & 5.71 & 34.29 \\
\hline \multirow[t]{6}{*}{ F32 } & 1 & 1 to 2 & (19) & 13.33 & 26.66 & 6 to 1 & (13) & 34.29 & 5.71 \\
\hline & 2 & 2 to 1 & (14) & 26.66 & 13.33 & 1 to 6 & (16) & 5.71 & 34.29 \\
\hline & 3 & 4 to 1 & (14) & 32.00 & 8.00 & 1 to 2 & (10) & 13.33 & 26.66 \\
\hline & 4 & 1 to 4 & (14) & 8.00 & 32.00 & 2 to 1 & (13) & 26.66 & 13.33 \\
\hline & 5 & 1 to 6 & (14) & 5.71 & 34.29 & 4 to 1 & (12) & 32.00 & 8.00 \\
\hline & 6 & 6 to 1 & (14) & 34.29 & 5.71 & 1 to 4 & (10) & 8.00 & 32.00 \\
\hline \multirow[t]{6}{*}{ F52 } & 1 & 1 to 4 & (21) & 8.00 & 32.00 & 2 to 1 & (13) & 26.66 & 13.33 \\
\hline & 2 & 4 to 1 & (15) & 32.00 & 8.00 & 1 to 2 & (15) & 13.33 & 26.66 \\
\hline & 3 & 6 to 1 & (14) & 34.29 & 5.71 & 1 to 6 & (10) & 5.71 & 34.29 \\
\hline & 4 & 1 to 6 & (17) & 5.71 & 34.29 & 6 to 1 & (13) & 34.29 & 5.71 \\
\hline & 5 & 1 to 2 & (14) & 13.33 & 26.66 & 4 to 1 & (12) & 32.00 & 8.00 \\
\hline & 6 & 2 to 1 & (24) & 26.66 & 13.33 & 1 to 4 & (10) & 8.00 & 32.00 \\
\hline \multirow[t]{6}{*}{$\mathrm{F} 22$} & 1 & 1 to 4 & (19) & 8.00 & 32.00 & 6 to 1 & (13) & 34.29 & 5.71 \\
\hline & 2 & 4 to 1 & (14) & 32.00 & 8.00 & 1 to 6 & (16) & 5.71 & 34.29 \\
\hline & 3 & 2 to 1 & (14) & 26.66 & 13.33 & 1 to 2 & (10) & 13.33 & 26.66 \\
\hline & 4 & 1 to 2 & (14) & 13.33 & 26.66 & 2 to 1 & (13) & 26.66 & 13.33 \\
\hline & 5 & 1 to 6 & (14) & 5.71 & 34.29 & 4 to 1 & (11) & 32.00 & 8.00 \\
\hline & 6 & 6 to 1 & (24) & 34.29 & 5.71 & 1 to 4 & (10) & 8.00 & 32.00 \\
\hline \multirow[t]{6}{*}{ F42 } & 1 & 1 to 2 & (19) & 13.33 & 26.66 & 4 to 1 & (13) & 32.00 & 8.00 \\
\hline & 2 & 2 to 1 & (14) & 26.66 & 13.33 & 1 to 4 & (16) & 8.00 & 32.00 \\
\hline & 3 & 6 to 1 & (14) & 34.29 & 5.71 & 1 to 6 & (10) & 5.71 & 34.29 \\
\hline & 4 & 1 to 6 & (17) & 5.71 & 34.29 & 6 to 1 & (13) & 34.29 & 5.71 \\
\hline & 5 & 1 to 4 & (14) & 8.00 & 32.00 & 2 to 1 & (12) & 26.66 & 13.33 \\
\hline & 6 & 4 to 1 & (16) & 32.00 & 8.00 & 1 to 2 & (19) & 13.33 & 26.66 \\
\hline \multirow[t]{6}{*}{ F62 } & 1 & 1 to 6 & (19) & 5.71 & 34. 29 & 2 to 1 & (13) & 26.66 & 13.33 \\
\hline & 2 & 6 to 1 & (21) & 34.29 & 5.71 & 1 to 2 & (19) & 13.33 & 26.66 \\
\hline & 3 & 4 to 1 & (14) & 32.00 & 8.00 & 1 to 6 & (10) & 5.71 & 34.29 \\
\hline & 4 & 1 to 4 & (17) & 8.00 & 32.00 & 6 to 1 & (20) & 34.29 & 5.71 \\
\hline & 5 & 1 to 2 & (16) & 13.33 & 26.66 & 4 to 1 & (14) & 32.00 & 8.00 \\
\hline & 6 & 2 to 1 & (24) & 26.66 & 13.33 & 1 to 4 & (16) & 8.00 & 32.00 \\
\hline
\end{tabular}

Note: The bracketed numbers are sessions required to meet the stability criteria. 
(13.33 s to $26.66 \mathrm{~s}), 2: 1,1: 4$ ( $8 \mathrm{~s}$ to $32 \mathrm{~s}), 4: 1,1: 6(5.71 \mathrm{~s}$ to $34.29 \mathrm{~s})$, and $6: 1$. The order of the delays for each subject is shown in Table 1.1. The location of the reinforcers alternated across conditions. When the small immediate reinforcer was associated with the left lever the houselight remained off; the houselight was lit when the small immediate reinforcer was associated with the right lever.

\section{Procedure}

\section{Phase I}

All subjects had previous experience with concurrent chain schedules so no pretraining was required. The subjects had previously spent nines months responding on an SI - LD paradigm. The three Ethanol Rats had only ever responded for $10 \%$ ethanol sucrose reinforcement. The Plain Rats had previously been reinforced with plain sucrose only. All sessions began with the levers retracted and the lights off. The front lights were turned on and the front levers were inserted into the operant chamber to signal trial start. Independent FI 10s FI 10s schedules then began timing and the subject was able to respond on either lever. The first response made by the subject after the FI $10 \mathrm{~s}$ elapsed was the choice response. After the choice response, the front levers retracted and the light for the unchosen lever extinguished.

The terminal link began immediately after the subject made a choice response. The light associated with the chosen lever remained on during the terminal link delay. When the terminal link delay had elapsed, the chosen lever was re-inserted into the operant chamber. The subject was then required to respond on the lever (FR 1) and reinforcer delivery immediately followed that response. If the choice response was for the SI alternative, the dipper located below the chosen lever delivered a single dip 
of reinforcer. After the SI reinforcer was delivered, the light above the dipper was turned off and the post-reinforcer blackout commenced. Choice of the LD alternative resulted in delivery of three dips of reinforcer from the dipper located under the chosen lever. After a LD reinforcer was delivered, the light associated with an LD choice was extinguished and a 45 -second inter-trial interval commenced. It took 10 seconds longer to deliver the LD reinforcer than it took to deliver the SI reinforcer (i.e. the total time to deliver each dip of reinforcer was 5 seconds). The SI postreinforcer blackout was equal to the difference of the LD delay and the SI delay plus the time it took to deliver the LD reinforcers. For example, during the 1:4 delay ratio conditions the SI terminal link delay was $8 \mathrm{~s}$ and the LD delay was $32 \mathrm{~s}$ thus the length of the post-reinforcer blackout for the SI condition was $34 \mathrm{~s}$. The postreinforcer blackout used following the SI reinforcer ensured trials were of equal durations and is standard for self-control paradigms (e.g. Rachlin \& Green, 1972). There was no post-reinforcer blackout for the LD alternative. Trials were separated by a $45-\mathrm{s}$ ITI.

On average, sessions ran five days a week. Training on each delay ratio condition continued until the stability criteria were met. The criteria were that a) the moving average proportion of responses to the SI alternative of the final five sessions was within 0.05 of the preceding five sessions, b) that criterion $a$ was met for two consecutive sessions and, c) there was no monotonic increasing or decreasing trend in the proportion of SI choices made during the last five sessions. 


\section{Phase II}

The experimental procedure for Phase II was identical to that of Phase I. The only exception was that all six subjects received ethanol sucrose for the SI reinforcer and plain sucrose for the LD reinforcer. That is, ethanol was removed from the LD reinforcer for the Ethanol Rats and ethanol was added to the SI reinforcer for the Plain Rats. The stability criteria used were the same criteria that were used for Phase I.

Results and Discussion

\section{Ethanol Consumption}

If ethanol sucrose is functioning as a reinforcer it will maintain operant responding. All subjects made responses that were reinforced with ethanol sucrose when it was available. The average ethanol consumption $(\mathrm{g} / \mathrm{kg} / \mathrm{hr})$ for each subject for each delay ratio condition is shown in Table 1.2. Only the subjects F12, F32 and F52 consumed ethanol sucrose in Phase I; all subjects consumed ethanol sucrose as a reinforcer in Phase II.

Table 1.2

Average ethanol consumption $(\mathrm{g} / \mathrm{kg} / \mathrm{hr})$ for each delay ratio condition for each Phase.

\begin{tabular}{lccccccc}
\hline & & $1: 2$ & $2: 1$ & $1: 4$ & $4: 1$ & $1: 6$ & $6: 1$ \\
\hline \hline Phase I & F12 & 1.45 & 1.81 & 1.65 & 1.65 & 1.08 & 0.85 \\
& F32 & 0.98 & 0.57 & 0.93 & 0.75 & 1.34 & 0.77 \\
& F52 & 0.56 & 1.08 & 0.58 & 0.60 & 0.71 & 0.73 \\
Phase II & & & & & & & \\
& F12 & 0.12 & 0.03 & 0.13 & 0.07 & 0.20 & 0.10 \\
& F32 & 0.21 & 0.29 & 0.50 & 0.27 & 0.48 & 0.48 \\
& F52 & 0.21 & 0.08 & 0.10 & 0.21 & 0.22 & 0.24 \\
& F22 & 0.03 & 0.10 & 0.21 & 0.24 & 0.13 & 0.28 \\
& F42 & 0.46 & 0.10 & 0.06 & 0.01 & 0.00 & 0.21 \\
& F62 & 0.02 & 0.04 & 0.05 & 0.07 & 0.10 & 0.06 \\
\hline
\end{tabular}


During Phase I, the Ethanol Rats consumed on average $1.01 \mathrm{~g} / \mathrm{kg} / \mathrm{hr}$ of ethanol (range 0.71 to $1.42 \mathrm{~g} / \mathrm{kg} / \mathrm{hr})$, which is greater than the metabolic rate of ethanol for rats $(0.30$ g/kg/hr, Wallgren \& Barry, 1970; as cited in Heyman \& Oldfather 1992). During Phase II, when ethanol delivery was contingent on SI choices, all subjects allocated some of their responding to the SI alternative. As can be seen in Table 1.2, there were no systematic effects of delay or quality on the consumption of ethanol. During Phase II, only one subject (F32) consistently consumed more ethanol $(\mathrm{g} / \mathrm{kg} / \mathrm{hr})$ than the metabolic rate of ethanol. None of the remaining subjects altered their proportion of SI choices to an extent that enabled them to maintain a pharmacologically significant ethanol intake during Phase II. Ethanol sucrose was clearly functioning as a reinforcer for the subjects in this experiment; however, during Phase II, the value of the SI (the ethanol sucrose alternative) was not great enough to increase the proportion of SI entries to a level at which consumption would have pharmacological effects. Nevertheless, all six subjects' behaviour changed when the quality of one of the reinforcer alternatives changed which indicates that behaviour distribution in this concurrent chains paradigm was a function of reinforcer quality.

\section{Phase I}

The data used for this analysis were the median data obtained from the last five sessions of each condition. During Phase I, one Plain Rat (F42) responded exclusively for the LD alternative at all delays. To enable quantitative models to be fit to the data, 1.0 was added to the median values of the obtained choice (i.e. entry) and response data for F42.

The first question of interest was whether there was any indication that the response distribution of the subjects whose behaviour was reinforced with ethanol sucrose was 
different from that of the subjects whose behaviour was reinforced with plain sucrose.

Figure 2.3 shows the proportion of SI responses made by each subject during Phase I, the proportions are averaged over all six conditions. Visual analysis of the data indicates that the Ethanol Rats tended to be more impulsive than the Plain Rats. The average proportion of SI responses made by the Ethanol Rats was 0.44 and for the Plain Rats it was 0.17.

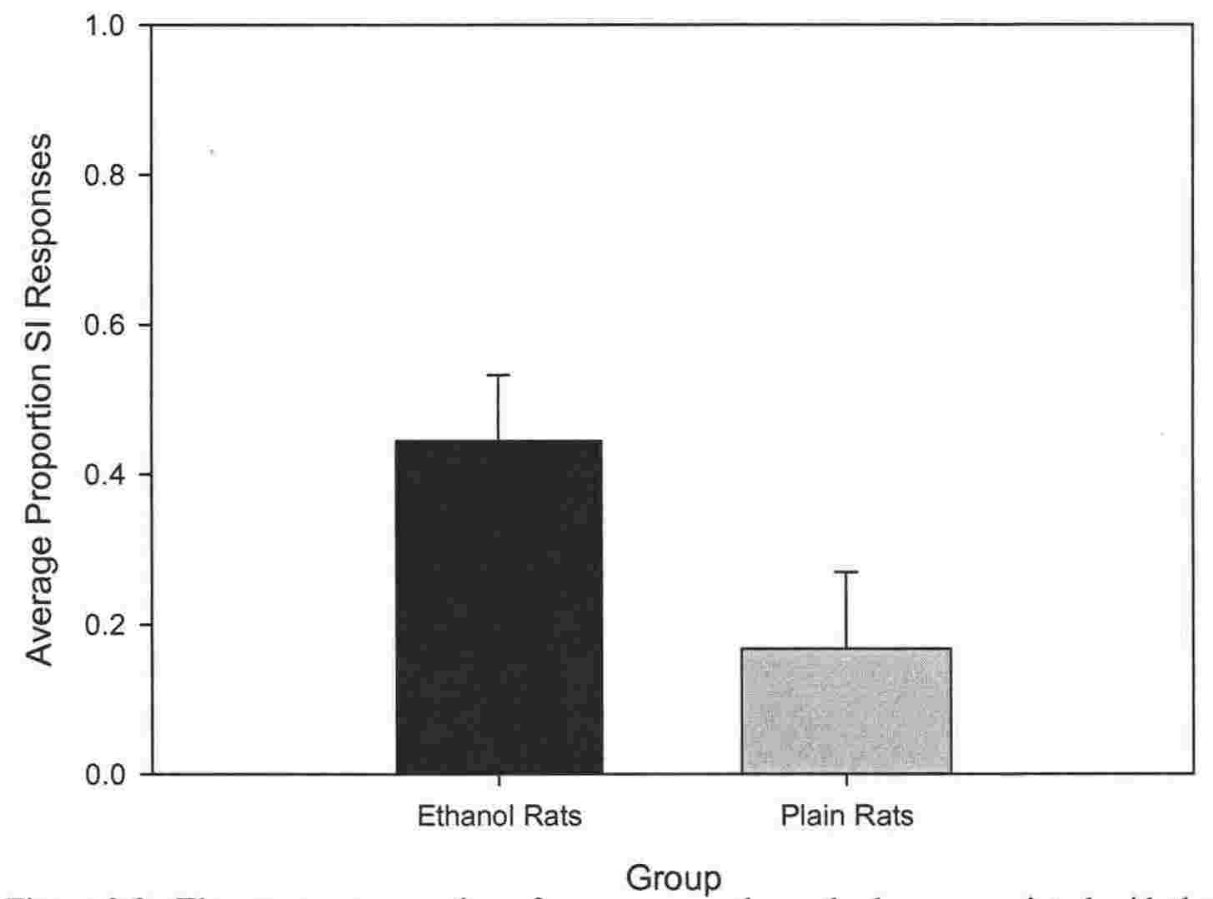

Figure 2.3. The average proportion of responses made on the lever associated with the impulsive alternative during Phase I. The dark bar is the average responses made by the three Ethanol Rats and the lighter bar is the average responses made by the three Plain Rats.

The next question was whether delay ratio had a systematic effect on behaviour.

Firstly, the proportion of SI responses made in Phase I (Figure 2.4) when the SI alternative was located on the left and when the SI alternative was located on the right were visually examined for evidence of contingency control. This examination of the individual subjects' behaviour indicated there were side biases. A side bias was present in the behaviour of all subjects except for F42 who responded exclusively to 

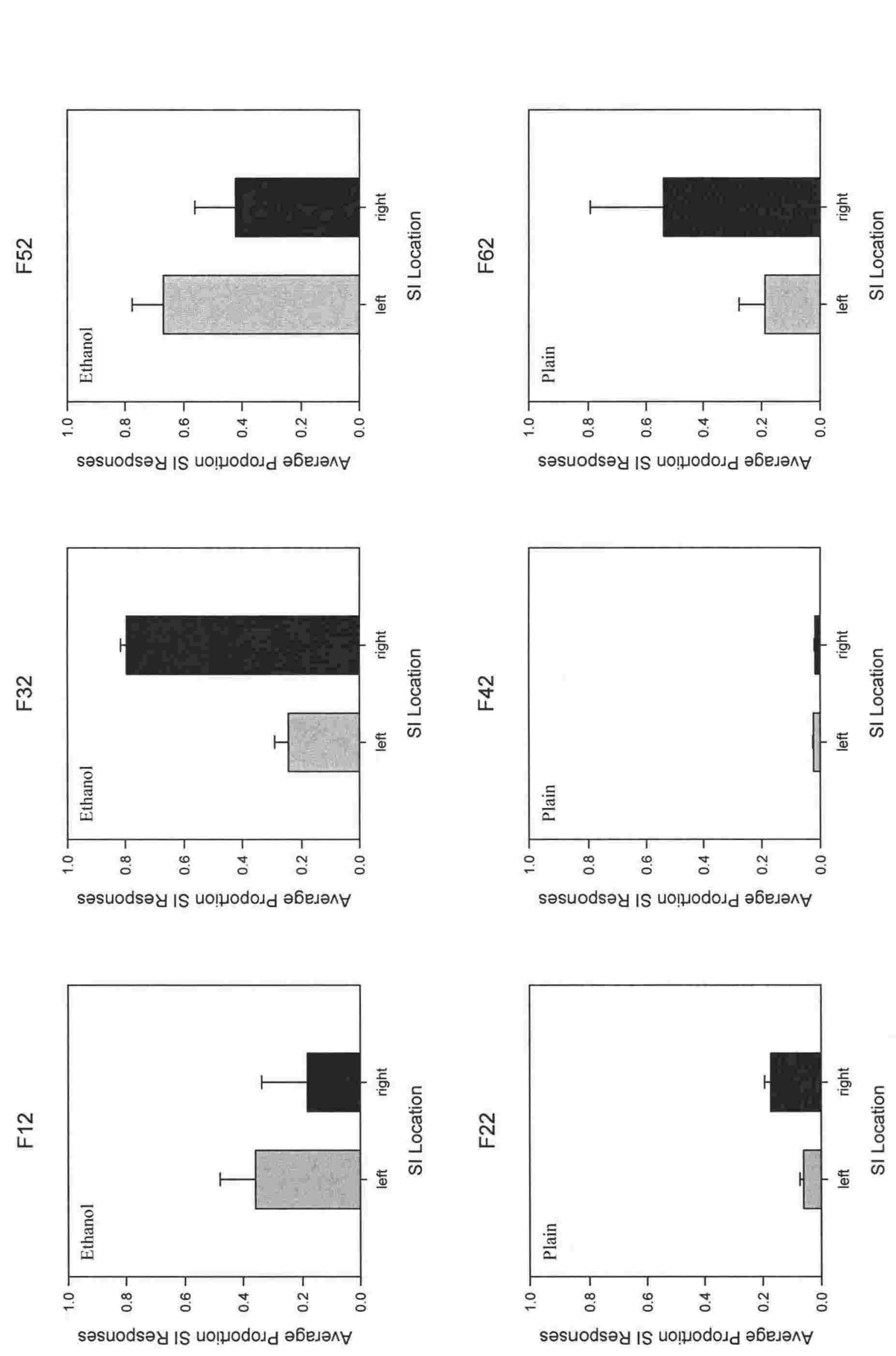

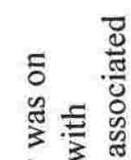

긍

이 3

吾

흥

क

등

ह

든

u도음

얼

호.

동

인을

吉 品

吾

등

흘 흥

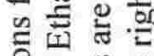

윯 䒕

i $-D$

$叉$ 总

政

可

$\cong$.

on

들

亏

运

는 잉

案声.

등 홍ㅇㅇ

क ప

ठ의

ㅎㅇㅇㅝ

명을

का $3 \cdot \frac{2}{2} \frac{\pi}{3}$

깅.

ㅇํㅇ

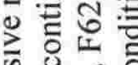

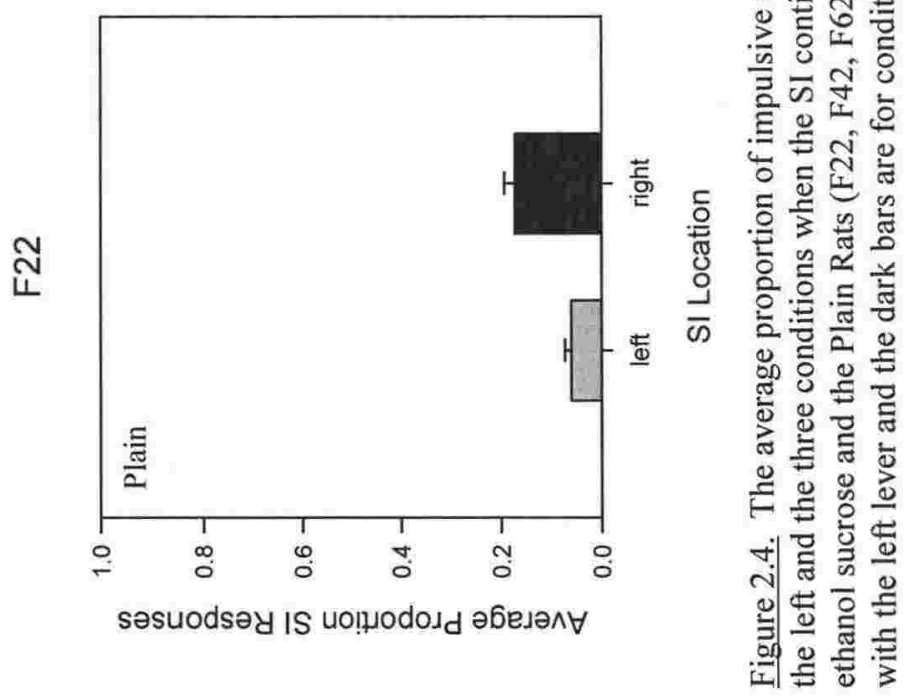


the self-controlled alternative at all delays irrespective of the location of the LD contingency. This can be seen visually in Figure 2.4 as the proportion of SI responses (i.e. the proportion of responses allocated to the SI initial link schedule) varied as the location of the SI contingency alternated between the left and the right lever. The individual proportion of responses made on the left alternative for each delay ratio condition can be seen in Appendix A.

To reduce the impact of side bias, the data was analysed by taking the median number of choices made for each contingency (SI or LD) combined over the final five sessions for each delay ratio condition and its reversal (Figure 2.5). The changes in behaviour as delay ratio changed during Phase I were consistent with previous literature and did not differ as a function of the quality of reinforcer. For two of the Ethanol Rats (F12 and F52) and two of the Plain Rats (F22 and F62), the proportion of SI choices (i.e. the proportion of entries into the SI alternative) increased as the delay ratio became extreme. Although it is of note that the increase in SI choices was not a monotonically increasing function for all of these rats, behaviour at the 1:6 delay ratio was consistently more impulsive then at the 1:2 ratio for these four subjects. This pattern of behaviour was similar to the one found by Green and Estle (2003) where choice between different amounts of food or water reinforcers was sensitive to changes in the delay ratio. In that study, choice of the SI alternative was also less frequent at the least extreme delay ratio as compared to the most extreme delay ratio.

The remaining two rats (F32 and F42) were insensitive to the delay ratios. F32 tended to be indifferent to the terminal link contingencies and the mean proportion of SI choices for that subject was 0.52 . F42 was consistently self-controlled and never 


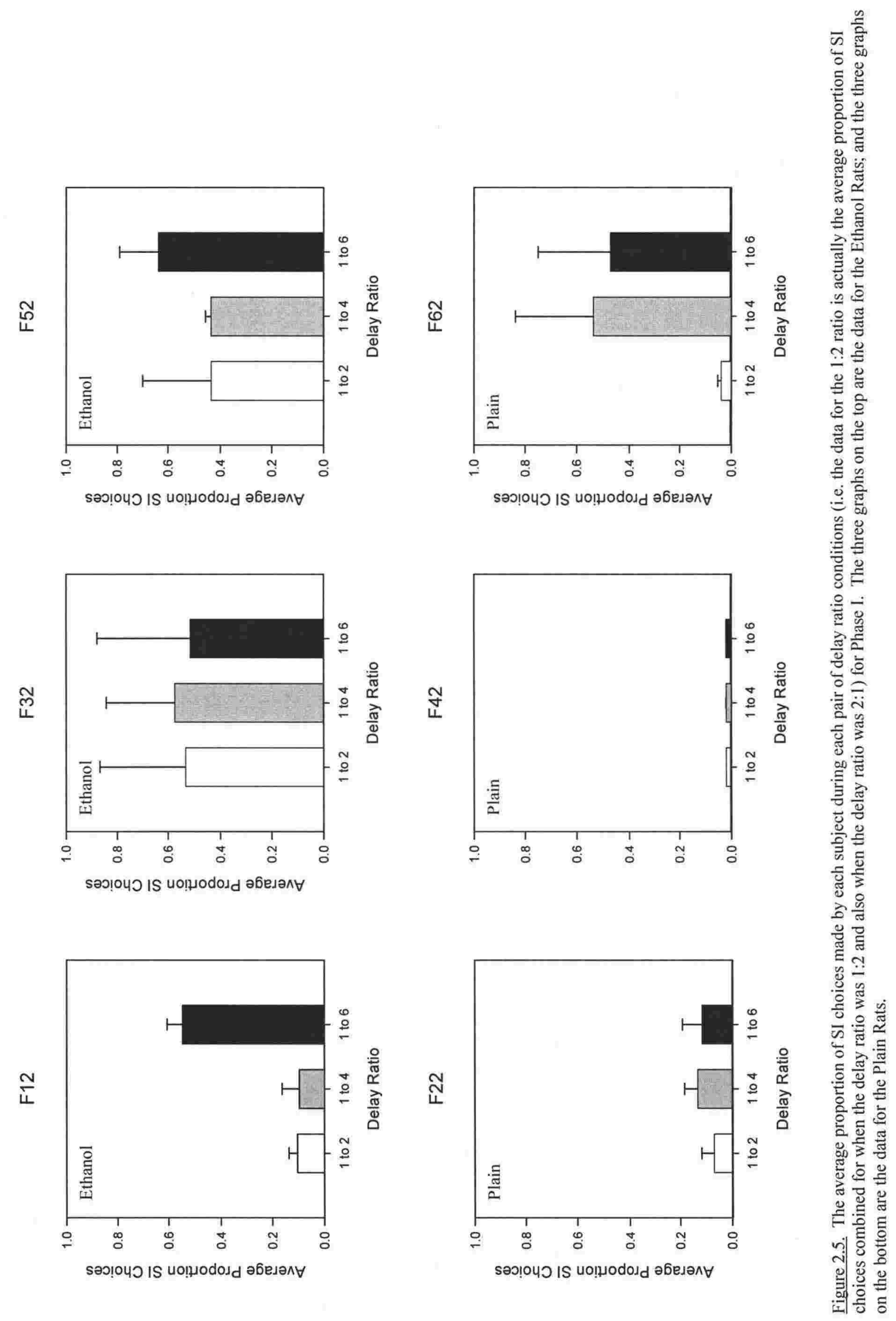


chose the SI alternative during Phase I. The lack of sensitivity to delay ratio demonstrated by these two rats may have resulted from the limited range of delays chosen for this study. In the Green \& Estle (2003) study the starting delay ratio was determined individually for each rat so that the subjects initially chose the SI at the most extreme delay ratio. The most extreme ratios ranged from 1:24 to 1:60 for the four rats in that study as compared to $1: 6$ in the present study. Similarly, in the original Rachlin and Green (1972) study there was individual variation in response distribution between reinforcer alternatives and subjects showed varying degrees of sensitivity to the reinforcer delay. Thus, the idiosyncratic impact of delay ratios on choice in the current study is consistent with previous literature.

\section{Phase II}

In Phase II reinforcer quality was manipulated and the behaviour of all the subjects was reinforced with ethanol sucrose for SI choices and with plain sucrose for LD choices. The individual proportion of responses made on the left alternative for each delay ratio condition is shown in Appendix B. The behaviour of the three Ethanol Rats became more orderly in Phase II (Figure 2.6). All three subjects monotonically increased responding on the SI alternative as the delay ratio became more extreme and thus entered the SI alternative more frequently. The choices made by the Plain Rats were not as orderly as that of the Ethanol Rats. The pattern of behaviour by F62 was similar to that of the Ethanol Rats in Phase II; however, F22 and F42 did not monotonically increase choices of the impulsive alternative as the delay ratio became more extreme. F22, however, made more choices that were impulsive at the 1:6 ratio than at the 1:2 ratio. Although F42 showed greater contingency control than in Phase I, its change in behaviour as a function of increasing delay ratio was the opposite to that observed in the other rats behaviour. This may have reflected an order effect with 


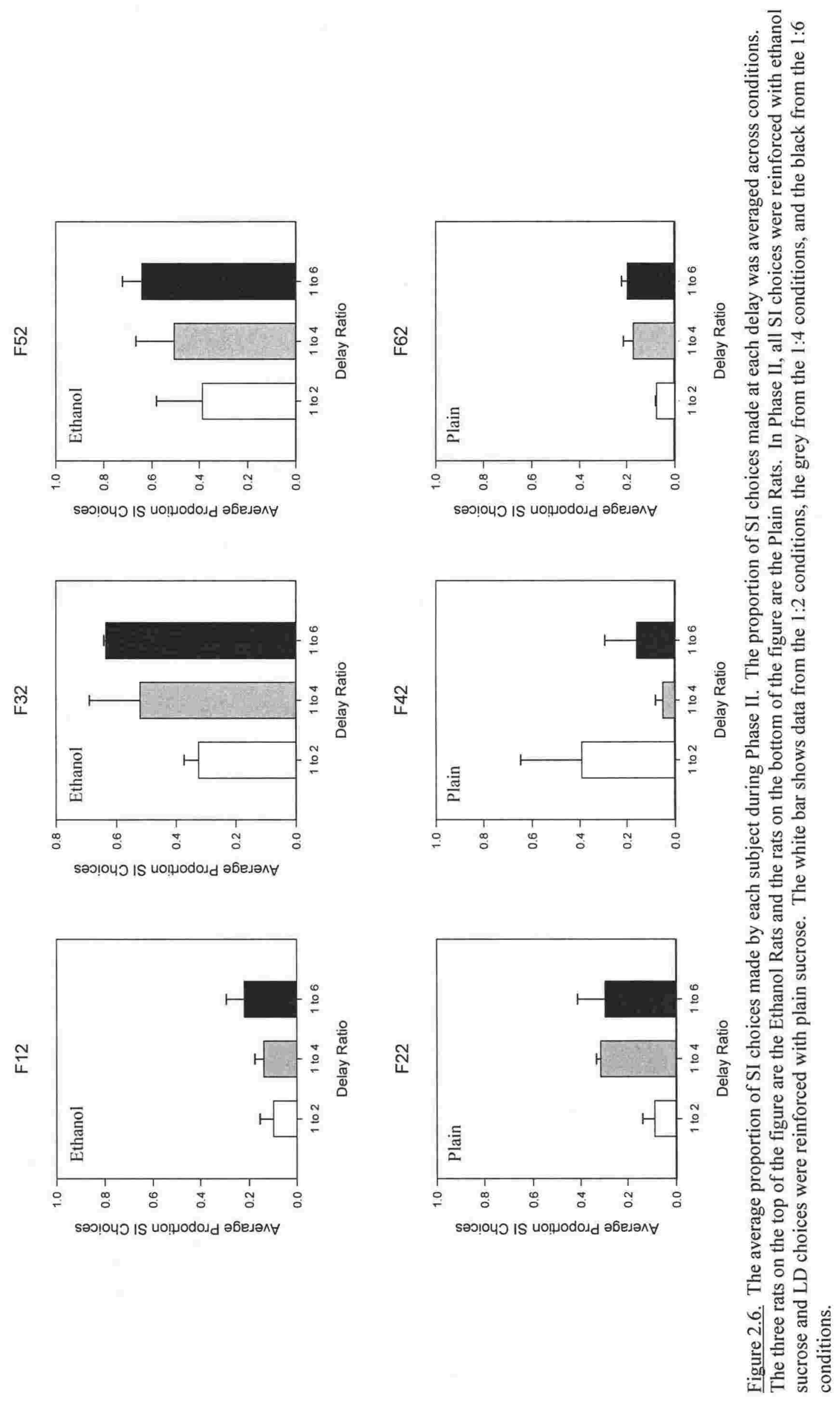


F42 responding on the SI alternative more frequently and thus entering the SI alternative more frequently with increased exposure to the different qualities contingency. For this subject the order of presentation of the delay ratios in this phase was 1:4, 1:6 and then $1: 2$ and across these conditions the rat showed a monotonic increase in SI choices as can be seen in Figure 2.6.

In general, reinforcing behaviour on each alternative with a different quality reinforcer seemed to result in a more consistent effect of reinforcer delay on choice. In Phase I only four of the six subjects had tendency to increase responding, and hence entries, for the SI reinforcer as the delay ratio became more extreme. This contrasts with the finding in Phase II that five subjects showed consistent increases in SI entries at the largest delay ratio as compared to the smallest delay ratio. Presenting subjects with concurrently available reinforcers of different qualities may have produced more discriminable alternatives. This would be consistent with the findings of Ettinger et al. (1981) when different types of grain were delivered on multiple schedules. That study found that behavioural contrast only occurred when the relative values of the types of grain delivered for the two alternatives were sufficiently different. Thus, the more orderly influence of delay on choice behaviour found in Phase II of the current study may be due to an increase in the discriminablity of the alternatives when choices were between different quality rather than identical quality reinforcers. 


\section{Model Fitting}

\section{The Generalised Concatenated Matching Law}

The next issue for Experiment 1 was whether the behaviour of subjects could be described by a generalised version of the concatenated matching law (Equation 10).

$\frac{B_{L}}{B_{R}}=b\left(\frac{D_{L}}{D_{R}}\right)^{a_{D}}\left(\frac{A_{L}}{A_{R}}\right)^{a_{A}}$

The sensitivity parameters for the Generalised Concatenated Matching Law (GCML) are $\mathrm{a}_{\mathrm{D}}$, sensitivity to the delay ratio; and $\mathrm{a}_{\mathrm{A}}$, sensitivity to the reinforcer amount ratio. Chelonis and Logue (1997) suggested that a greater sensitivity to reinforcer amount (i.e. $a_{A}>a_{D}$ ) is associated with more choices that are self-controlled and a greater sensitivity to reinforcer delay (i.e. $\mathrm{a}_{\mathrm{A}}<\mathrm{a}_{\mathrm{D}}$ ) will result in behaviour that is more impulsive. If this holds true then subjects that make more choices that are impulsive should have a smaller sensitivity ratio $\left(a_{A} / a_{D}\right)$ than subjects that have make more selfcontrol choices. As the sensitivity ratio increases control by the reinforcer amount ratio increases and subjects should make fewer choices of the SI alternative.

Microsoft Excel Solver was used to estimate the parameter values for $b, a_{D}$, and $a_{A}$ by maximising the percentage variance accounted for by the model when the obtained proportion of SI entries was compared to the predicted proportion of SI entries (Grace, 1994). Data from the conditions when the SI contingency was associated with the left lever and when it was associated with the right lever were not averaged for model fitting. The free parameter values, percent variance accounted for $(\% \mathrm{VAC})$, the sensitivity ratio $\left(\mathrm{a}_{\mathrm{A}} / \mathrm{a}_{\mathrm{D}}\right)$ and the average proportion of SI choices made by each subject for Phase I are shown in Table 2.1. Sensitivity to amount was greater than sensitivity to delay, which corresponds to the tendency for subjects to be self- 


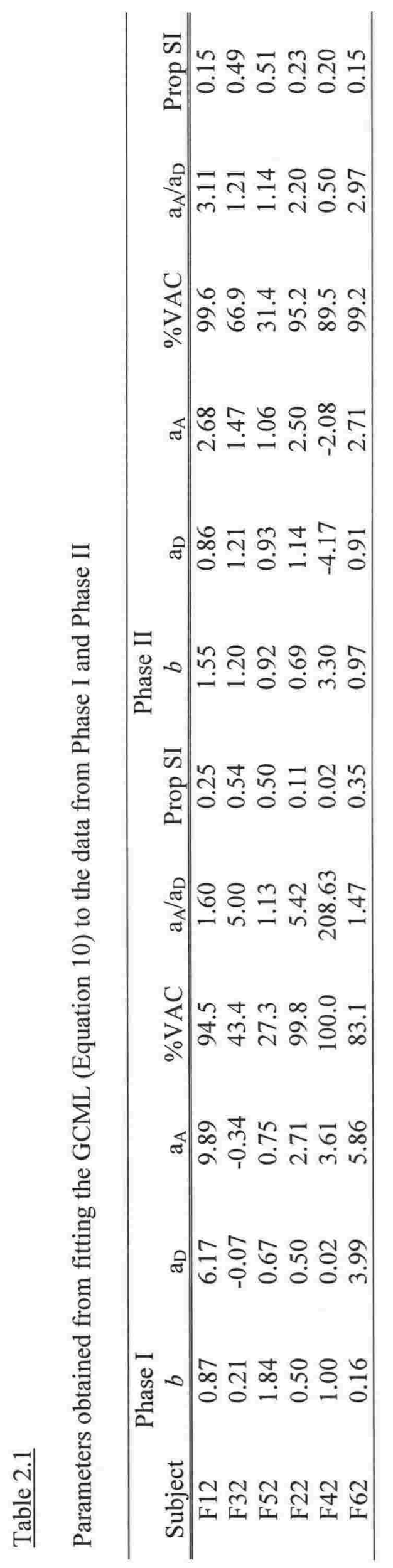


controlled. The average variance accounted for by the generalised concatenated matching equation was $74.7 \%$ (range $27.3 \%$ to $100.0 \%$ ). It is of note that the poor fit (27.3\%) obtained for F52 that has orderly data in Figure 2.6 results from the side bias which confounded contingency bias (i.e. bias towards either the SI or the LD contingency, see Appendix A). The derived parameters for Phase II are shown in Table 2.1. The average variance accounted for in Phase II of the experiment was $80.3 \%$ (range $31.4 \%$ to $99.6 \%$ ). When the sensitivity parameter values obtained from Phase I were compared to the values in Phase II there were no systematic changes in either sensitivity to delay or sensitivity to amount (Figure 2.7).
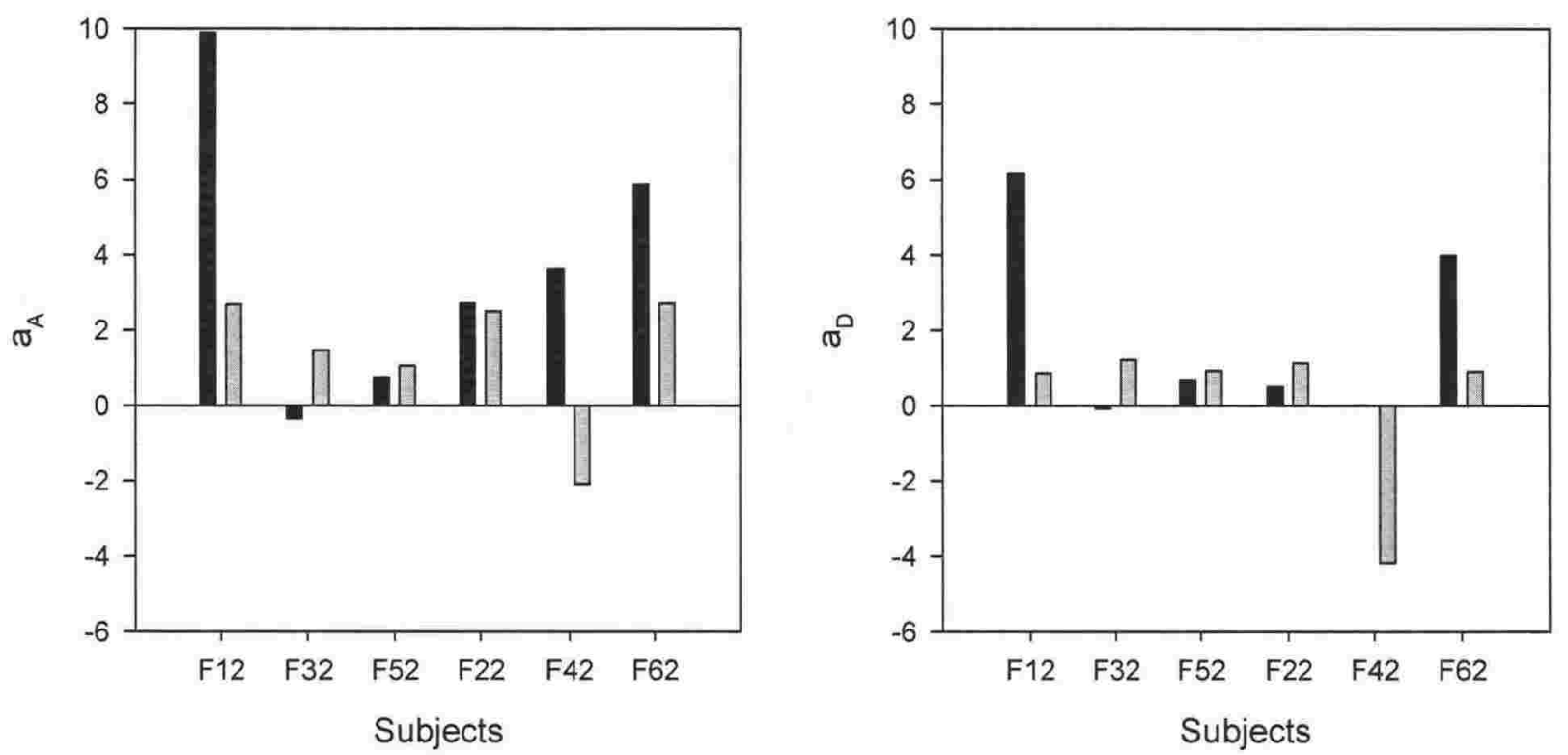

Phase I Phase II

Figure 2.7. Derived values for the sensitivity parameters. The graph on the left is sensitivity to the reinforcer amount ratio and the graph on the right is the sensitivity to reinforcer delay ratio. The values were derived by fitting the generalised concatenated matching law (Equation 10) using the proportion of SI choices made for all delay ratio conditions. The data from conditions in which the SI contingency was on the left and when it was on the right were not combined

The next step was to consider changes in the sensitivity ratio $\left(a_{A} / a_{D}\right)$ when the reinforcer quality was changed in Phase II as compared to Phase I. Changes in the sensitivity ratio should be reflected in changes in the proportion of SI choices made 
by each subject. If $\mathrm{a}_{\mathrm{A}} / \mathrm{a}_{\mathrm{D}}=1$ the programmed ratios of amount and delay should control behaviour equally and subjects should tend to be self-controlled at the smallest delay ratio (1:2) and become increasingly impulsive as the delay ratio increased. If $\mathrm{a}_{\mathrm{A}} / \mathrm{a}_{\mathrm{D}}$ increases in Phase II the influence that the delay ratio (relative to the influence of the amount ratio) has on choice should increase. That is, increases in $a_{A} / a_{D}$ (in Phase II $v s$. Phase I) should be reflected in subject's behaviour as a decrease in impulsive choices in Phase II; and decreases in $\mathrm{a}_{\mathrm{A}} / \mathrm{a}_{\mathrm{D}}$ should result in an increase in impulsive choices. This relationship between the sensitivity ratio and choice behaviour was true for four of the six subjects (Figure 2.8); an increase in $a_{A} / a_{D}$ was associated with a decrease in impulsive choices for two subjects (F12, F62) and a decrease in $a_{A} / a_{D}$ was associated with an increase in impulsive choices for two subjects (F22, F42). While this was not particularly convincing, the sensitivity ratios were negatively correlated with the average proportion of SI choices in both Phase I
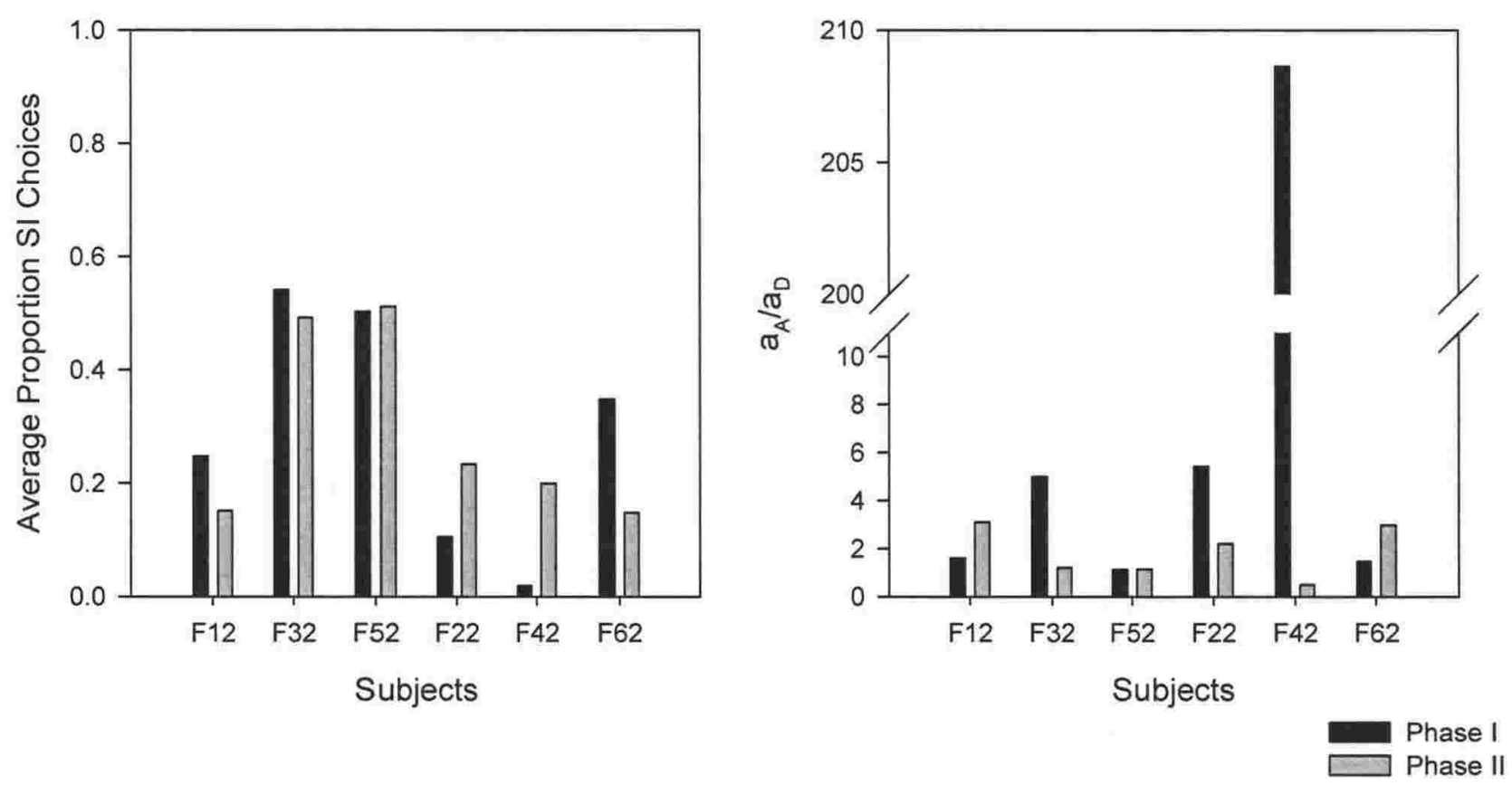

Figure 2.8. The graph on the left shows the average proportion of SI choices that subjects made over all six delay ratio conditions for Phase I and Phase II. The right hand graph shows the sensitivity ratio for each subject. The dark bars are data from Phase I and the light bars are data from Phase II. 
$\left(r_{\mathrm{s}}=-0.66\right)$ and Phase II $(\mathrm{r}=-0.58)$, which indicates that behaviour became more impulsive as the sensitivity ratio decreased.

\section{The Contextual Choice Model}

An alternative model of choice behaviour that typically accounts for more of the data variance in concurrent chain paradigms than Equation 10 is the CCM (Grace, 1994). The CCM (Equation 11) takes the temporal context of the programmed schedules into account. Another important feature of CCM is that it addresses the potential confound of changes in sensitivity to amount as delay to reinforcer delivery increases.

$$
\frac{B_{L}}{B_{R}}=b\left(\frac{R_{L}}{R_{R}}\right)^{a_{R}}\left[\left(\frac{D_{R}}{D_{L}}\right)^{a_{D}}\left(\frac{A_{L}}{A_{R}}\right)^{a_{A}}\right]^{\left(\frac{T_{i}}{T_{i}}\right)}
$$

For instance, White and Pipe (1987) found that sensitivity to reinforcer amount increased when the absolute delay to terminal link reinforcer delivery increased. This is a problem for the generalised concatenated matching law, as it requires sensitivity to the terminal link ratios to remain constant. In that study, increasing delay to reinforcer delivery also increased the duration of the terminal link. In such a case, CCM predicts that increasing terminal link duration $\left(\mathrm{T}_{\mathrm{t}}\right)$ increases sensitivity to amount by increasing the sensitivity exponent $\left(\mathrm{a}_{\mathrm{A}}\left(\mathrm{T}_{\mathrm{t}} / \mathrm{T}_{\mathrm{i}}\right)\right)$. CCM states that sensitivity to terminal link variables increases as the duration of the terminal link increases (if the initial link durations are unchanged between conditions) because increases in terminal link duration increases the value of the ratio (Tt/Ti) (Grace, 1999).

For the current study, trial duration varied as the delay ratio varied, thus the influence that the terminal link contingencies had on initial link responding should have increased as the terminal link duration increased relative to the initial link duration. Therefore, for the current study, the values of the sensitivity parameters derived using 
the GCML may have been confounded by the effect of context. The CCM model was therefore fit to the data from Phase I and Phase II. CCM predicted that preference for the less delayed terminal reinforcer would increase as the duration of the terminal link increased in relation to the duration of the initial link. As the delay ratio became more extreme, the contextual exponent $\left(\mathrm{T}_{\mathrm{t}} / \mathrm{T}_{\mathrm{i}}\right)$ increased and it was predicted that behavioural distribution in the initial links would become more impulsive (i.e. the SI would be chosen more frequently). The terminal link entry ratio $\left(R_{s i} / R_{l d}\right)$ was not experimentally controlled so the obtained entry ratio was used and sensitivity to the entry ratio $\left(a_{R}\right)$ was set equal to 1 (Grace, 1999). CCM accounted for more variance in the data than did GCML. The average variance accounted by CCM for Phase I was $94.9 \%$ (range $75.2 \%$ to $100.0 \%$ ) and $96.7 \%$ (range (91.6\% to $99.8 \%$ ) for Phase II. Although these fits were an improvement to the fits obtained using the generalised concatenated matching equation a caveat to this approach is that (as noted by Grace, 1999) the use of the obtained entry ratio effectively places an dependent variable on both sides of the CCM equation. A reasonable fit of the equation to the data was therefore expected because the terminal link entry ratio was controlled by the initial link response ratio. The analysis was conducted, however, to determine if accounting for the influence of changing terminal link duration between conditions would result in more orderly effects on the sensitivity parameters. The derived parameter values and the sensitivity ratios are shown in Table 2.2. Reinforcer quality was ethanol sucrose for the first three subjects (F12, F32, F52) and plain sucrose for the final three subjects (F22, F42, F62). The seventh and final columns show the average proportion of impulsive choices (Prop SI) each subject made averaged over the six delay ratio conditions used in each phase. There were no systematic changes to the sensitivity parameter values when reinforcer quality was manipulated in Phase II (Figure 2.9). 


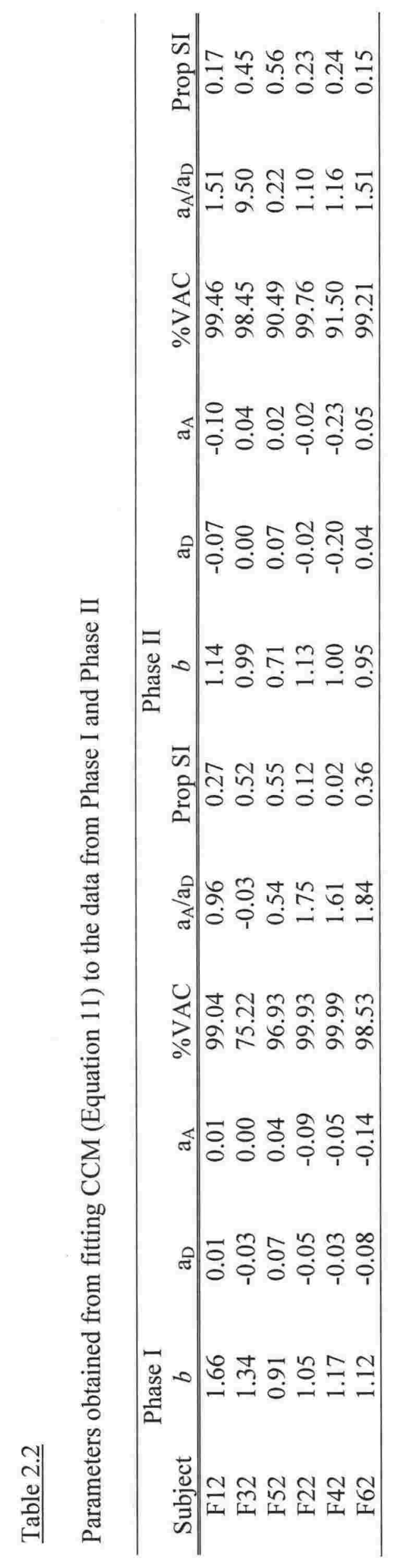


When the parameter values derived from the CCM were compared to those derived using the GCML it was clear that the values are much smaller for CCM. This overall decrease in the sensitivity parameter values probably resulted from using the obtained terminal link entry rate to fit the model.
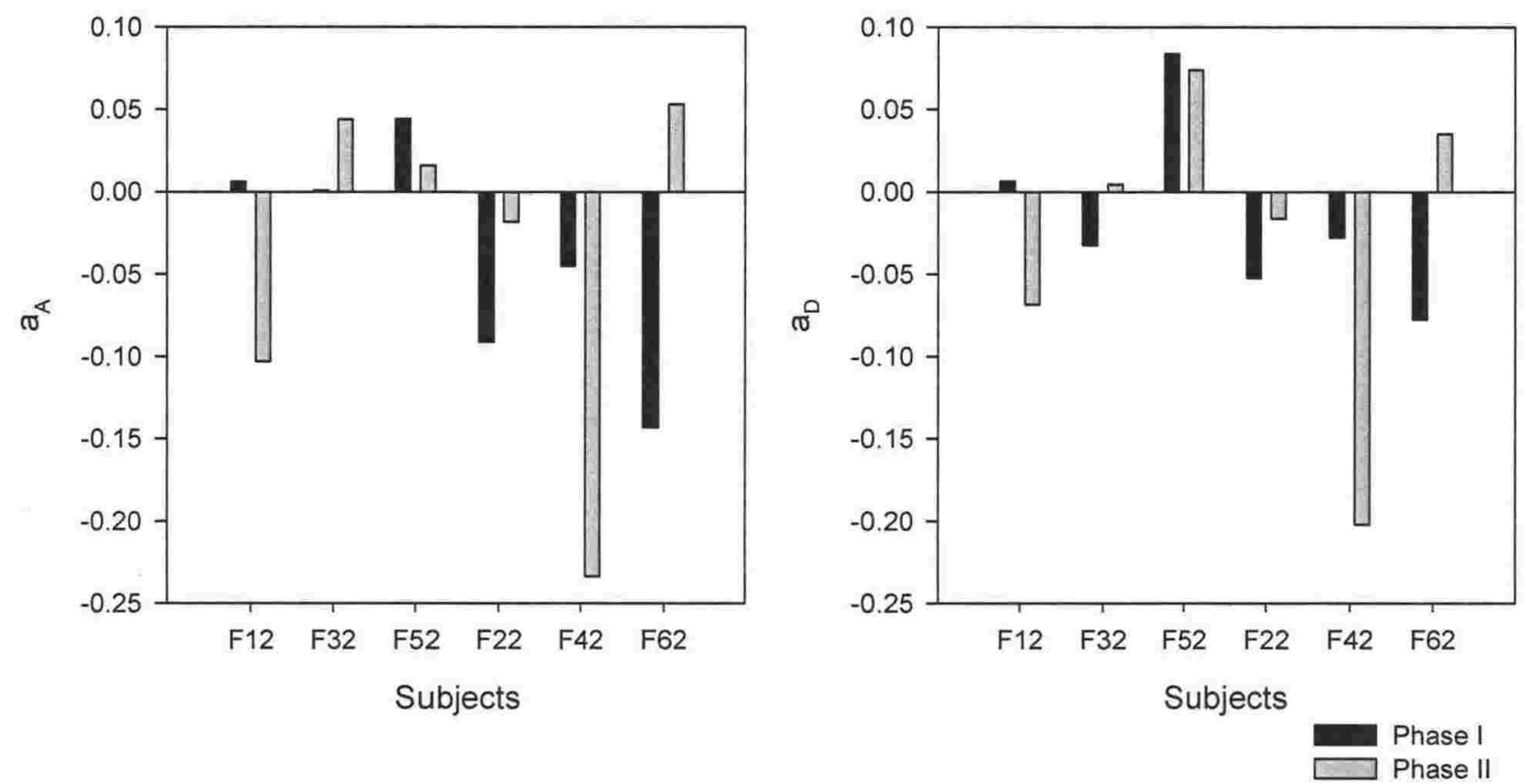

Figure 2.9. The derived values for sensitivity to the reinforcer amount ratio $\left(a_{A}\right)$ and for sensitivity to the reinforcer delay ratio $\left(a_{D}\right)$. Values were derived using Equation 11. Phase I data are depicted by the dark bars and Phase II data are depicted by the lighter bars.

The left side graph in Figure 2.10 shows the proportion of SI responses made by each subject during Phase I and Phase II. The graph on the right shows the sensitivity ratios $\left(a_{A} / a_{D}\right)$ for each subject. Similar to the generalised concatenated matching analysis, increases in the average proportion of SI responses made in Phase II versus Phase I should be reflected in a decrease in the sensitivity ratio. Likewise, decreases in the proportion of SI responses in the second phase should result in an increase in the sensitivity ratio. This relationship was accurate for five of the six subjects, the subject that did not meet this prediction was F62 who decreased SI responding in 
Phase II but had a smaller sensitivity ratio in Phase II $\left(\mathrm{a}_{\mathrm{A}} / \mathrm{a}_{\mathrm{D}}=1.51\right)$ as compared to

Phase I $\left(a_{A} / a_{D}=1.84\right)$. Once again, a negative correlation was found between the derived sensitivity ratios and proportions of SI choices made by each subject. For Phase I $r=-0.75$ and for Phase II $r_{\mathrm{s}}=-0.35$.
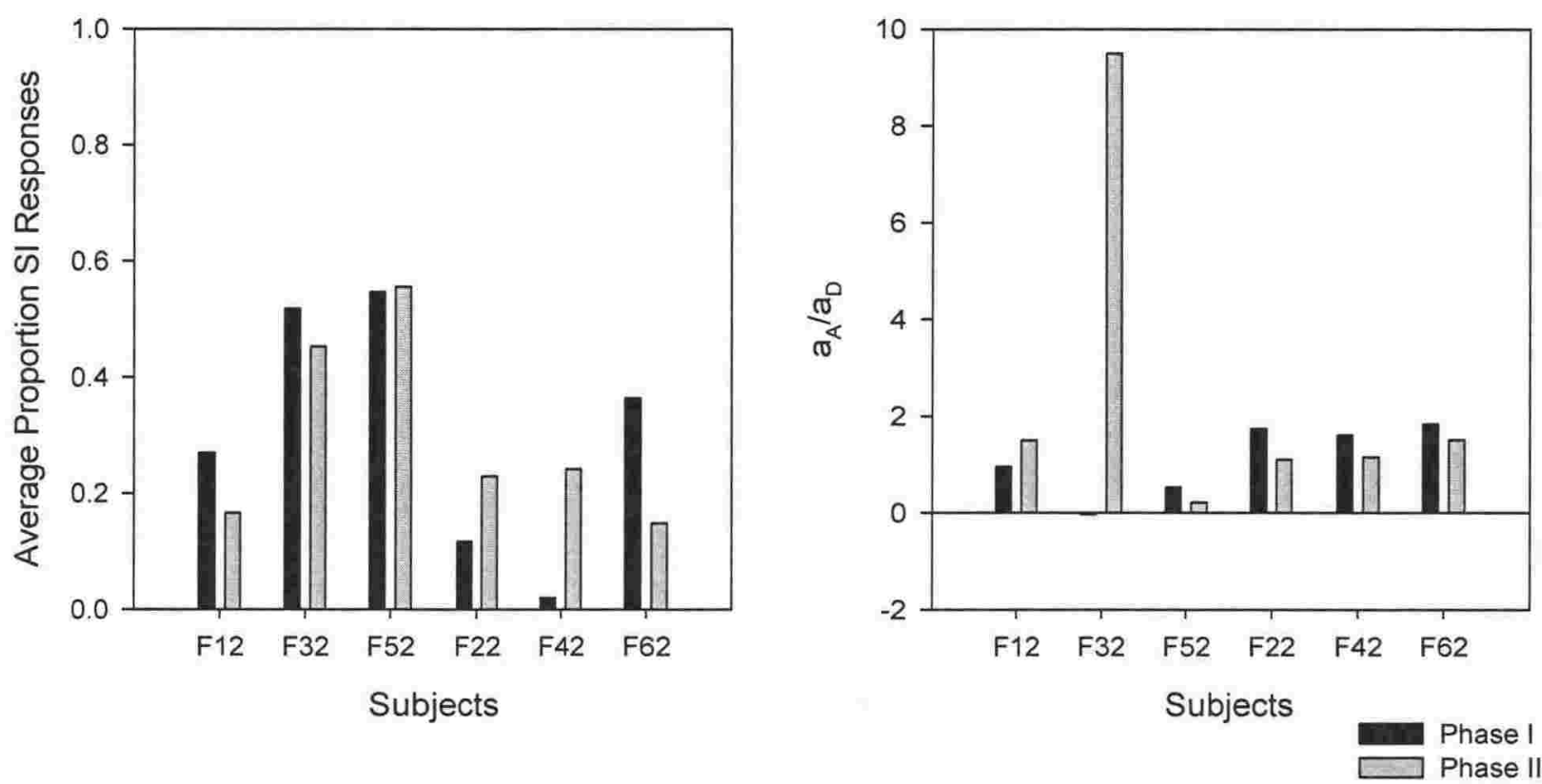

Figure 2.10. The average proportion of SI responses made by each subject for Phase I and Phase II is shown in the left hand graph and the sensitivity ratios are shown in the right hand graph. Decreases in the proportion of SI responses were predicted to correspond to increases in the sensitivity ratio. Likewise, increases in the proportion SI responses were predicted to correspond to decreases in the sensitivity ratio. The dark bars are data from Phase I and the light bars are data from Phase II.

When the parameter values derived using the CCM were compared to those derived from the GCML it was apparent that there was more individual variation in the parameter values from the former equation. Since the impact of the experimental contingencies is more consistent when the GCML, rather than CCM, is fit to the data; and because of the issue of having a dependent variable on both sides of the CCM equation, it is more parsimonious to use GCML to describe the data obtained in this experiment. 
The results from this experiment are well described by the GCML and the derived changes in the sensitivity parameters are theoretically sound. The impact of changing reinforcer quality in this study was that the reinforcer contingencies were more salient when the alternatives delivered different, rather than identical, quality reinforcers. That is, changes in the delay ratio were more salient when the two alternatives delivered qualitatively different reinforcers. Thus, this traditional self-control paradigm was able to model choice between qualitatively different reinforcers. Similar to studies that hold quality constant but vary delay to reinforcer delivery, the current study found that behaviour became more impulsive as the delay ratio became more extreme.

One of the advantages of Experiment 1 was that it used different quality reinforcers, which meant that the experimental manipulation was a more realistic analogue of human behaviour. However, there is an aspect to self-control behaviour in the "real world" that is not taken into account using this traditional self-control paradigm. Outside the laboratory setting, additional primary reinforcers are often obtained immediately following a choice (i.e. at a point analogous to the end of the initial link of concurrent chains paradigms) as well as after a delay. For example, a person trying to reduce their weight by dieting must sometimes chose between competing immediate reinforcers such as a piece of fruit and a piece of cake. Each of those immediate reinforcers is associated with a different delayed reinforcer; the fruit is associated with weight loss and the cake is associated with weight gain. That is, there are two differences between behaviour in non-experimental choice situations and experimental analogues of self-control: (i) there is often an immediate reinforcer associated with the self-controlled as well as the impulsive alternative; and (ii) the two 
immediate reinforcers differ qualitatively. The novel self-control paradigm used in Experiment 2 examined the impact that the addition of qualitatively different immediate reinforcers had on choice behaviour. 


\section{EXPERIMENT 2}

\section{Alternative Self-Control Paradigms}

Although many studies examining self-control use concurrent-chains procedures that are similar to the one used in Experiment 1, other paradigms have been used. The most frequently used paradigms incorporate variations of the SI versus LD procedure. These variations include titration procedures and fading procedures. In delay titration procedures one alternative remains available after a constant delay while the delay for the competing alternative is systematically adjusted to find the point at which subjects are indifferent and chose each alternative equally often (e.g., Mazur, 1988). The resulting indifference points are used to calculate the rate of hyperbolic decay of reinforcer value, which allows the phenomenon of amount-dependent temporal discounting to be studied. A variation of the titration procedure is also used where the amount of one reinforcer alternative is held constant while the amount of the alternative reinforcer is adjusted, again the purpose is to identify the indifference points and use those points to calculate rate of reinforcer value discounting (e.g., Mitchell \& Rosenthal, 2003). Fading procedures also look at responding for competing SI and LD reinforcer alternatives. Conditions begin with equal delays to both alternatives, which results in preference for the alternative that delivers the larger amount of reinforcer. The delay to the smaller reinforcer is then gradually decreased while the delay to the larger reinforcer is held constant (or conversely the delay to the smaller reinforcer is held constant as delay to the larger reinforcer is increased). This fading of the delay results in a tendency to maintain preference for the LD over a greater range of delays than would be predicted without using the procedure (e.g., Logue et al., 1984). Fading paradigms show that experience choosing large delayed reinforcers can increase self-control. Although the paradigms discussed contribute to 
the understanding of the mechanisms underlying choice behaviour, there is an aspect of self-control that they do not address.

Traditional SI - LD self-control paradigms do not address situations in which an individual has the opportunity to choose between competing immediately available reinforcers each having different long term consequences. Rachlin (1995) refers to choices between immediately available reinforcers as choices between local acts and the long-term effects of those choices as global patterns. According to Rachlin (1995), behaving with self-control depends on an organism repeating local acts that forfeit immediate gain in order to maintain desirable global patterns. Of particular importance for this thesis are self-control paradigms that consider the impact that contingent global (delayed) reinforcers have on preference for competing local (immediate) reinforcers. There are some studies in the human literature that address the competing local acts and global patterns posited by Rachlin (1995). As an example, Warry et al. (1999) examined human response distribution between concurrently available local reinforcers (short term outcome) each having a different effect on the global reinforcement rate (long term outcome). The local choice was between two different point values: The value of Option A was always less than the value of Option B. However, choosing Option B reduced the total amount of points that could be earned during the session; while choosing Option A increased the global reinforcement rate. Subjects tended to choose Option B even when information was provided to make the long-term consequences of each local choice more salient. However, there was evidence that as the subjects gained experience with the reinforcement contingencies they chose Option A more frequently. That is, local choice behaviour was affected by experience with global reinforcer contingencies. 
The Warry et al. (1999) study indicates that people's choices are not only controlled by isolated SI and LD reinforcers but also by global contingencies associated with local choices. Immediately available reinforcers may differ in terms of such things as magnitude or quality and (when considering self-control situations) one of the immediate alternatives is more valuable than the other alternative. Furthermore, delayed reinforcers, which are each exclusively linked to one of the immediate reinforcers, may also differ in such elements as immediacy, magnitude, and quality. For instance, a recovering alcoholic will frequently need to choose between consuming ethanol-containing and ethanol-free beverages. In relation to short-term outcomes, selecting an ethanol-free drink (such as soda) will be less valuable than an ethanol-containing one (such as scotch). However, soda and scotch are associated with different long-term (delayed) consequences. Drinking soda adds to the recovering alcoholic's newly established pattern of sobriety, whereas choosing scotch returns them to the old behaviour pattern of alcoholism (Rachlin, 1995, 2000).

Assuming sobriety is more valuable to the individual than continued alcoholism, and that sobriety depends on the selection of ethanol-free beverages, the terms impulsiveness and self-control are redefined for such situations. Impulsiveness, in this context, is forfeiting the more valued delayed reinforcer (sobriety) by choosing the more valuable immediate reinforcer (scotch). Self-control, again in this context, is forfeiting the more valued immediate reinforcer (scotch) to gain access to the more valued delayed reinforcer (sobriety). Another way to term this is that impulsivity is choosing to have more value now (MN) and self-control is choosing to have more value later $(\mathrm{ML})$. A More Now - More Later $(\mathrm{MN}-\mathrm{ML})$ paradigm was developed to study such situations for this thesis. The advantage of the MN - ML paradigm is that 
it enables investigation of self-control situations that are not modelled by existing paradigms.

The principal differences between the current MN - ML paradigm and previous selfcontrol analogues that use concurrent chain schedules were that primary reinforcement was delivered in both the initial link and terminal link, and that different quality reinforcers were associated with the impulsive and self-controlled alternatives. The impact of initial link reinforcers on response allocation has been investigated when reinforcer quality is equal. Davison and Smith (1986) used concurrent schedules to investigate the impact that initial reinforcers had on response distribution between the alternatives when each option had a different terminal reinforcer rate. They found that adding initial link reinforcers made subjects insensitive to the terminal reinforcement contingencies. The procedure used by Davison and Smith differed from the procedures typically used to study self-control behaviour. Most self-control paradigms are arranged so that there is a fixed delay to terminal link reinforcement and the procedure Davison and Smith used delivered reinforcers in the terminal link according to a VI schedule. Thus, reinforcers in the terminal link were delivered after variable delays. There is evidence that variable delays to terminal link reinforcement effects initial link response distribution differently than fixed delays. For instance, (Grace, 1995) found that having variable delays to terminal reinforcement made sensitivity to reinforcer magnitude greater than sensitivity to reinforcer delay which is the opposite to findings from studies that use fixed delays to terminal link reinforcers (e.g., Chelonis \& Logue, 1997). Additionally, terminal link reinforcers were available for only 30 seconds and some of the VI schedules exceeded that delay (e.g. in one condition terminal reinforcers were 
delivered on VI 17-s versus VI 120-s schedules) therefore some terminal link entries would not have provided additional reinforcers (e.g., Moore, 1982). In contrast, in typical self-control paradigms primary reinforcement is delivered for each terminal link entry. The MN - ML paradigm adds initial link reinforcers while keeping other aspects of the concurrent schedules similar to those used in traditional SI - LD selfcontrol analogues making it easier to compare the results with those from existing self-control research.

In the current study, the initial link reinforcers were of the same amount but differed qualitatively. Quality was manipulated by adding ethanol to diluted sweetened condensed milk. MN initial reinforcers contained ethanol and ML initial reinforcers were ethanol-free. Ethanol was used to define the $\mathrm{MN}$ initial reinforcer as it was assumed that the ethanol-containing reinforcers were more valuable than equal magnitudes of ethanol-free reinforcers (hence the subjects would be impulsive when they chose to have more value now, $\mathrm{MN}$ ). The terminal reinforcers differed only in amount the $\mathrm{MN}$ alternative delivered 1 dip of terminal reinforcement while the $\mathrm{ML}$ alternative delivered 3 dips of reinforcement. That is, subjects were self-controlled when they responded to receive more value later $(\mathrm{ML})$ and would receive a greater total volume of reinforcer for that trial than they would have received had they chosen the $\mathrm{MN}$ alternative. Experiment 2 had three phases. Phase I examined whether prolonged experience consuming ethanol in one setting influenced responding for ethanol in a second setting. For Phase I, half of the subjects were pre-exposed to ethanol in their home cages prior to testing in the operant chambers. Responding to 
the MN - ML self-control paradigm was tested in Phase II ${ }^{1}$ and Phase III. For Phase II, MN was defined as the impulsive choice and responding to receive $\mathrm{MN}$ delivered one dip of ethanol milk in the initial link (the initial reinforcer) and one dip of plain milk in the terminal link (the terminal reinforcer). Responding to receive the selfcontrolled (ML) alternative was reinforced with one dip of plain milk in the initial link followed by three dips of plain milk in the terminal link. The delays to the two terminal reinforcement alternatives were identical within conditions but varied across conditions. The initial link contingencies and the terminal reinforcer amounts remained the same for Phase III but all choices were reinforced with ethanol milk in the terminal link. The terminal reinforcer quality was changed in Phase III so that the impact of reinforcer quality on initial link responding could be examined.

For all conditions, the ratio of the delays to the terminal reinforcers was 1:1 but the absolute duration of the delays varied. It can be assumed that if the subjects were given the choice of receiving either one dip of milk or three dips of milk immediately they would choose the alternative that delivered the larger amount of reinforcement (Anderson \& Woolverton, 2003; Rachlin \& Green, 1972). Snyderman (1983) investigated the effect of absolute delay on preference in concurrent chains. He found that when the delay ratio was held constant at 1:1 but the absolute delay durations were increased preference for the larger reinforcer amount increased. Ito and Asaki (1982) also found that subjects tended to increase preference for the larger reinforcer amount when the delay ratio was 1:1 and the absolute duration of the delays increased. These findings are consistent with human data indicating that the rate of

\footnotetext{
${ }^{1}$ Phase II of this study was published in the Journal of Experimental Psychology: Animal Behavior Process, vol. 1, 2004.
} 
temporal discounting depends on the amount of the reinforcer with larger reinforcers discounting at a slower rate.

Although amount dependent discounting is frequently found in the human literature (Johnson \& Bickel, 2002; Myerson \& Green, 1995) little support has been found for the existence of amount dependent discounting in rats (e.g., Green et al., 2004; Mitchell \& Rosenthal, 2003). A study into magnitude effects on discounting using pigeons and rats was conducted by Green et al. (2004), they concluded there was no systematic evidence of amount dependent discounting. However, the fitted functions to the data of two of the four rats in that study show a monotonic increase in the discount rate as the amount of reinforcement increased. The results of studies in which equal delays to terminal reinforcer delivery lead to an increase in preference for the larger reinforcer, combined with the results of Green et al., suggest that amount dependent discounting may exist in rats.

If there is amount dependent discounting of the terminal link reinforcers, the effect of increasing delays to the terminal link reinforcers on preference will depend on whether behaviour is sensitive to the initial and/or terminal link reinforcement contingencies. If only the delayed terminal reinforcers control initial link behaviour we would expect to see preference shift in the direction of the alternative that delivers the larger terminal reinforcers. If subjects' initial link responding is only influenced by the local reinforcer contingencies behaviour should not change as the delay to the terminal reinforcers is increased. Finally, if initial link responding is controlled by both the initial link and terminal link reinforcers then as the terminal link delays are increased control by the terminal link reinforcer should decrease and control by the 
initial link reinforcer increase. In this case, then behaviour should shift in the direction of the preferred initial link reinforcer as delay to the terminal link increases.

Method

\section{$\underline{\text { Subjects }}$}

Sixteen male Norway-hooded rats maintained at $85 \%$ of their individual free feeding weights served as subjects. Subjects were fed once daily to maintain target weights. Housing conditions were the same as for Experiment 1. Subjects were assigned to one of two groups. The subjects in the pre-exposed group $(n=8)$ were given access to ethanol in their home cages and the rats in the non-exposed group ( $\mathrm{n}=8)$ drank only water. One pre-exposed subject developed an inner ear infection and was replaced with a naïve rat that completed the graduated acclimatisation procedure (explained below).

\section{Apparatus}

Two standard operant chambers were used in this experiment. Each chamber was equipped with three levers. There was one fixed lever centred on the back wall of the chamber and two retractable levers on the front wall. The retractable levers were 18 $\mathrm{cm}$ from the chamber floor and $16.5 \mathrm{~cm}$ apart. A red light was positioned $3.5 \mathrm{~cm}$ above each of the front levers. The fixed lever was centred on the rear wall $6.5 \mathrm{~cm}$ above the chamber floor and a red light was positioned $5.0 \mathrm{~cm}$ above this lever. A liquid dipper was located $12 \mathrm{~cm}$ below each of the front levers. The dipper located below the lever associated with the ML contingency delivered plain milk; the dipper below the lever associated with the MN contingency delivered ethanol milk. 


\section{$\underline{\text { Procedure }}$}

\section{Phase I-Pre-exposure to Ethanol}

The pre-exposure phase of this experiment was designed to investigate if experience consuming ethanol in a free access situation would affect operant responding for ethanol-containing and ethanol-free reinforcers that were available on competing schedules. A graduated acclimatisation procedure (Schenk, Gorman, \& Amit, 1990) established ethanol as a reinforcer. The target ethanol concentration was $10 \%$ and the vehicle used for pre-exposure was tap water. Pre-exposure occurred 7 days per week for 4 weeks.

Pre-exposure occurred in subjects' home cages. Two $100 \mathrm{ml}$ plastic sipper bottles were hung on the wire mesh approximately $5 \mathrm{~cm}$ from the front of each cage (one bottle was on the left and one on the right). Both bottles were always filled with water for the non-exposed subjects. Fresh water was supplied daily and the positions of the bottles alternated each day. Pre-exposed rats' bottles were either both filled with water or one bottle held water and the other a solution of ethanol in water (ethanol water). Ethanol water was available for the pre-exposed subjects on alternating days (that is, on Day 1 one bottle contained ethanol water and the other did not, on Day 2 both bottles contained water and so on). The position of the bottle containing ethanol alternated on ethanol days so that half of the time ethanol water was in the left-side bottle and half of the time ethanol water was in the right-side bottle. Volume consumed from each bottle was recorded every 24 hours.

Pre-exposed subjects were initially given a solution of $2 \%$ ethanol water. The ethanol concentration was then increased by $1 \%$ each ethanol day if a subject consumed at least $5 \mathrm{ml}$ of ethanol water within a 24-hour period. However, if a subject had 
consumed less than $5 \mathrm{ml}$ of ethanol water after 24 hours of access the concentration of the ethanol water was decreased by $1 \%$ on the next ethanol day. When that subject consumed at least $5 \mathrm{ml}$ of the lower concentration the sequence of increasing concentrations resumed on the following ethanol day. The entire sequence of $2 \%$ to $10 \%$ could be completed in 17 days.

Once a subject was consuming at least $5 \mathrm{ml}$ of $10 \%$ ethanol water in a $24 \mathrm{hr}$ period the acclimatisation period ended and ethanol was available in the home cage daily for one hour. At that time, the solution vehicle was changed to the reinforcer to be used during the operant paradigm (that is, the vehicle was switched to diluted sweetened condensed milk) and subjects were given free access to that reinforcer in their home cages. The pre-exposed subjects received one hour of free access to $10 \%$ ethanol in diluted sweetened condensed milk (ethanol milk) and non-exposed subjects were given one hour of free access to plain diluted sweetened condensed milk (plain milk).

\section{Pre-Training}

Pre-training began on Day 31. Subjects were trained to press the two front levers using a standard autoshaping procedure. Pre-exposed rats were reinforced with ethanol milk and non-exposed rats were reinforced with plain milk. The maximum possible volume of reinforcer obtained in each session was $4.8 \mathrm{ml}$ ( $96 \times 0.05 \mathrm{ml}$ dips). Training sessions lasted a maximum of 45 minutes. After each session, subjects were returned to their home cages where they were given 45 minutes of free access to the appropriate reinforcer (ethanol milk for pre-exposed subjects and plain milk for nonexposed rats). This post-session access to the reinforcer was intended to ensure the pre-exposed subjects continued to maintain their daily $\mathrm{g} / \mathrm{kg}$ ethanol intake. 
After subjects were consistently responding on the left and right levers post-session free access was discontinued and the response requirements were changed to concurrent variable interval (VI) schedules. Over several days, the schedules were increased from concurrent VI $15 \mathrm{~s}$ VI $15 \mathrm{~s}$ to concurrent VI $30 \mathrm{~s}$ VI $30 \mathrm{~s}$ and finally concurrent VI $60 \mathrm{~s}$ VI $60 \mathrm{~s}$. The subjects were finally taught to respond on the back lever using a chaining procedure in which a back lever response was reinforced by access to the front levers. Responding on the front levers was followed by delivery of the primary reinforcer appropriate for each subject. Experimental sessions began when all of the pre-exposed and non-exposed subjects responded reliably on all three levers.

\section{Phase II Plain Milk as Terminal Reinforcer}

\section{Reinforcement contingencies}

Reinforcement contingencies (Figure 3.1) were scheduled so that subjects could receive either a) a small amount of reinforcer immediately and a second small amount after a delay (More Now, MN); or b) a small amount of reinforcer immediately and a larger amount of reinforcer after a delay (More Later, ML). The immediate reinforcers were equal in magnitude but qualitatively different. The $\mathrm{MN}$ immediate reinforcer was ethanol milk and the $\mathrm{ML}$ immediate reinforcer was plain milk. The delayed reinforcers were of unequal magnitude (one dip versus three dips of reinforcer) and equal quality (plain milk). The delays to the terminal link were constant within a condition. The delays used were $0 \mathrm{~s}$ and $6 \mathrm{~s}$. The first condition completed was the 6-s delay condition and the $\mathrm{MN}$ reinforcer was associated with the left lever; this condition took an average of 23 sessions to complete. Next, the positions of the MN and ML contingencies were reversed and the 6-s delay condition 


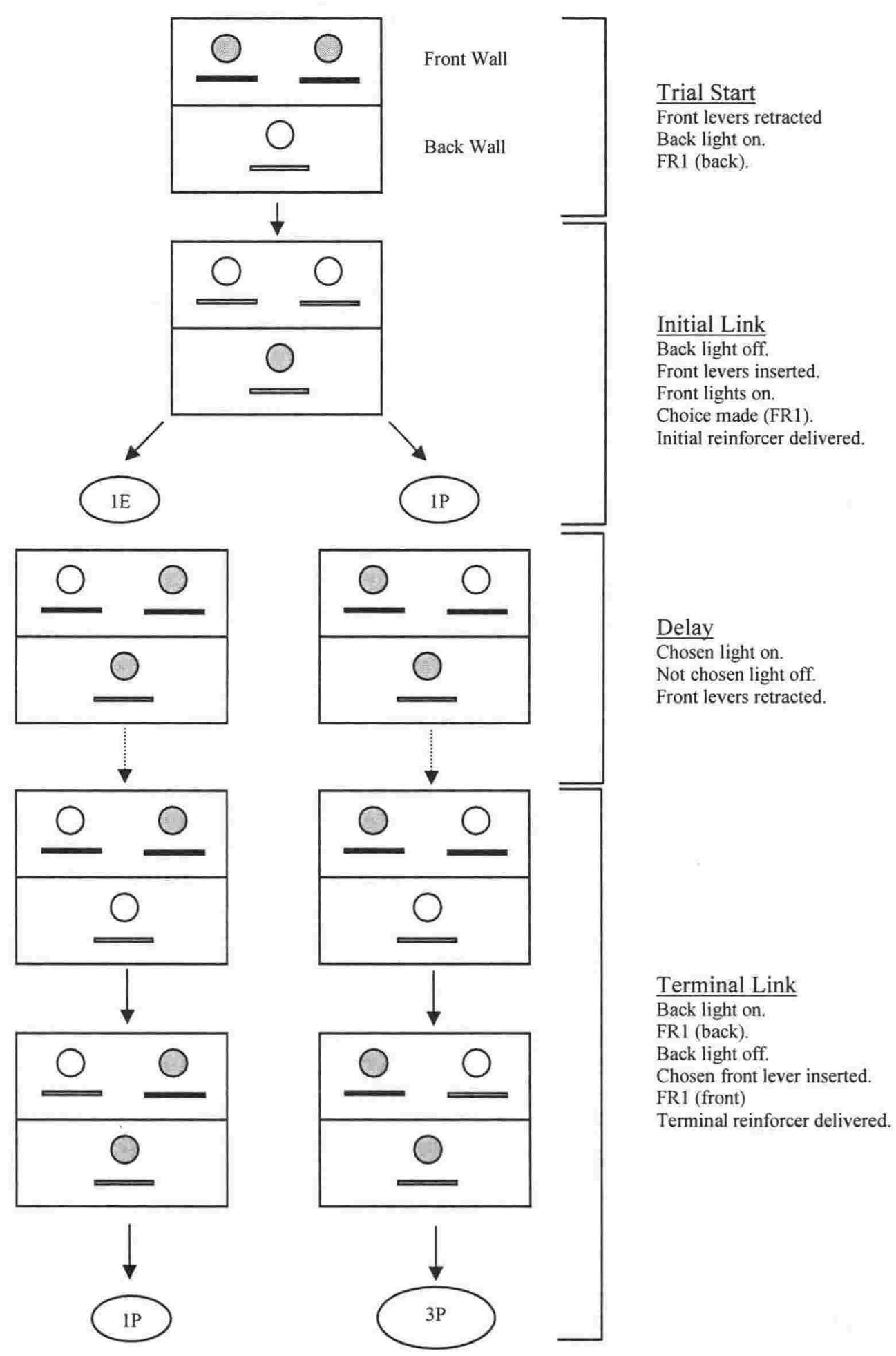

Figure 3.1. The novel self-control paradigm developed for this thesis. The boxes represent the operant chamber walls. The top rectangle of each box is the front wall and the bottom one is the back wall. Filled circles and bars indicate lights are off and levers are retracted. White lights and bars are lights that are on and levers that are inserted into the chamber. The label $\mathrm{P}$ indicates the reinforcer was plain milk and the label $\mathrm{E}$ indicates ethanol milk. At trial start, the front levers were retracted and the front lights off. The back light was on and a response on the back lever started the trial. Next; backlight off, the front lights on, and the front levers inserted into the chamber for the initial link. A response on one of those levers was the choice response and was immediately reinforced with one dip of the appropriate reinforcer. The inter-trial delay began timing from the choice response. The delay was the same for both alternatives. When the delay elapsed the back light turned off and FR1 turned it off and inserted the chosen lever. A response on that lever retracted it and turned off the front light. This was followed by delivery of the appropriate terminal reinforcer which completed the trial. Trials were separated by a 10 -s intertrial interval. 
was repeated with the $\mathrm{MN}$ reinforcer on the right, which took an average of 22 sessions to complete. Then the 0 -s delay conditions were completed, initially the $\mathrm{MN}$ contingency was located on the right and then it was located on the left. Both of these two conditions took an average of 11 sessions to complete. Each condition was run until the stability criteria had been met. The criteria were that the five session moving average proportion of $\mathrm{MN}$ choices made for the most recent five sessions had to be with 0.05 of the moving average for the previous five sessions; and that a minimum of 10 sessions had been completed.

More-Now or More Later Procedure

\section{Forced Choice Trials}

All sessions began with all lights off and the levers retracted. Ten forced choice trials were completed at the beginning of each session. Five of the forced choice trials were for the MN contingency and five were for the ML contingency. Turning on the back light signalled the beginning of each trial. A response on the back lever (FR 1) started a forced choice trial and illuminated either the left or the right light and inserted the associated lever into the chamber. A single response on the front lever resulted in the lever being retracted and was immediately reinforced with one dip of the appropriate reinforcer from the dipper located directly below the lever (i.e. one dip of ethanol milk for MN forced choices or one dip of plain milk for ML forced choices). After the initial link reinforcer was delivered the delay to the terminal link commenced. The light associated with the initial link choice remained on during the delay. After the delay elapsed, the front light was turned off and terminal link availability was signalled by illuminating the back light. A response on the back lever extinguished the light and led to delivery of the terminal reinforcer. The terminal reinforcer was 
one dip of plain milk for each MN initial link choice and three dips of plain milk for each ML initial link response. A 10 -s inter-trial interval (ITI) followed delivery of the ML terminal reinforcer. To keep trial lengths equal, the $\mathrm{MN}$ terminal reinforcement was followed by a $6 \mathrm{~s}$ blackout and then a $10 \mathrm{~s}$ ITI. The forced choice trials were included to ensure that subjects experienced both of the reinforcement alternatives.

\section{Free Choice Trials}

Free choice trials were identical to the forced choice trials except that both of the reinforcement contingencies were available during the initial link of each trial (Figure 3.1). After the subject responded on the back lever (to start a trial), the two front levers were inserted into the chamber and the two front lights were illuminated. A single response to either the left or the right lever was followed by delivery of the initial link reinforcer, next the delay, and then finally delivery of the terminal link reinforcer. Sessions lasted until subjects had completed 80 trials or until 45 minutes had elapsed, whichever occurred first.

\section{Phase III - Ethanol Milk as Terminal Reinforcer}

The procedure used for Phase II of Experiment 2 was the same as the procedure used in Phase I with a single exception. Initial link reinforcement was still plain milk for ML choices and ethanol milk for MN choices; however, both MN and ML choices were reinforced with ethanol milk in the terminal link instead of with plain milk. The no delay conditions were completed first. Initially, the $\mathrm{MN}$ contingency was associated with the right lever (average 15 sessions to complete). Then the contingencies reversed and that condition took an average of 19 sessions to complete. Finally, the two delay conditions were completed. First, the MN contingency was associated with the left lever which took 19 sessions to complete; then it was linked to 
the right lever which took 22 sessions to complete. The stability criteria were the same as those used for Phase II.

Results

\section{Pre-exposure}

Four subjects completed the acclimatisation sequence in 17 days. The remaining four subjects took $21,23,25$, and 29 days to reach the criterion of consuming at least $5.0 \mathrm{ml}$ of ethanol water in a 24-hour access period. By the final day of home cage exposure to ethanol, the pre-exposed rats consumed an average of $0.96 \mathrm{~g} / \mathrm{kg}$ of ethanol in a 24hour period (range $0.63 \mathrm{~g} / \mathrm{kg}$ to $1.10 \mathrm{~g} / \mathrm{kg}$ ). All eight rats that were pre-exposed to ethanol consumed more ethanol water than plain water during the final 24-hour exposure (Table 3.1). This indicated that subjects preferred the ethanol water solution to the plain water solution when it was freely available in their home cages.

\section{Table 3.1}

Ethanol water and plain water consumption by the pre-exposed rats for the final 24-hr free access period

\begin{tabular}{rccccc}
\hline Sub & Eth water $(\mathrm{ml})$ & Water $(\mathrm{ml})$ & Prop Eth & Eth $(\mathrm{ml})$ & g/kg ethanol \\
\hline \hline 31 & 36 & 6 & 0.86 & 3.6 & 1.10 \\
32 & 34 & 18 & 0.65 & 3.4 & 0.92 \\
33 & 38 & 9 & 0.81 & 3.8 & 0.93 \\
34 & 38 & 8 & 0.83 & 3.8 & 1.00 \\
41 & 40 & 2 & 0.95 & 4.0 & 1.09 \\
42 & 20 & 18 & 0.53 & 1.8 & 0.63 \\
43 & 36 & 2 & 0.95 & 3.6 & 1.05 \\
44 & 34 & 8 & 0.81 & 3.4 & 0.81 \\
\hline
\end{tabular}

More Now - More Later Paradigm

\section{Ethanol Consumption}

Three of the non-exposed rats had to be euthanised for reasons unrelated to the experiment, and did not complete the study. All data from those subjects are excluded from the analysis. The data used for analysis for Experiment 2 were the mean number 
of times the impulsive (MN) contingency was selected and of the mean number of times the self-controlled (ML) contingency was selected over last five sessions of each condition. The $85 \%$ target body weights for each subject were used to calculate average $\mathrm{g} / \mathrm{kg} / \mathrm{hr}$ intake of ethanol (Table 3.2). The first eight subjects in the table are the subjects that were pre-exposed to ethanol and the remaining five subject were not pre-exposed.

\section{Table 3.2}

Ethanol consumption (average $\mathrm{g} / \mathrm{kg} / \mathrm{hr}$ ) for the individual subjects

\begin{tabular}{ccccc}
\hline Subject & $\begin{array}{c}\text { Terminal Reinforcer Plain Milk } \\
\text { 0-s Delay }\end{array}$ & 6-s Delay & \multicolumn{2}{c}{ Terminal Reinforcer Ethanol Milk } \\
\hline 31 & 1.02 & 1.20 & 3.94 & 0-s Delay \\
32 & 0.74 & 0.79 & 3.56 & 3.94 \\
33 & 0.75 & 0.74 & 3.15 & 3.02 \\
34 & 1.08 & 1.16 & 3.20 & 3.76 \\
41 & 0.81 & 0.88 & 2.02 & 2.43 \\
42 & 1.35 & 1.56 & 4.81 & 4.05 \\
43 & 0.57 & 1.14 & 4.42 & 4.48 \\
44 & 0.51 & 1.31 & 3.94 & 4.31 \\
Mean & 0.85 & 1.10 & 3.63 & 3.70 \\
Std Dev & 0.28 & 0.28 & 0.86 & 0.68 \\
& & & & \\
35 & 0.10 & 0.27 & 4.48 & 4.64 \\
36 & 0.56 & 1.06 & 4.14 & 3.75 \\
45 & 0.97 & 1.11 & 3.18 & 3.17 \\
47 & 0.76 & 1.21 & 4.08 & 3.48 \\
48 & 1.05 & 1.19 & 3.50 & 3.57 \\
Mean & 0.69 & 0.97 & 3.88 & 3.72 \\
Std Dev & 0.38 & 0.39 & 0.52 & 0.55 \\
\hline
\end{tabular}

Terminal Reinforcer Quality

The pre-exposed subjects consumed more ethanol $(\mathrm{g} / \mathrm{kg} / \mathrm{hr})$, on average, than the nonexposed rats for both of the delay conditions when the terminal reinforcer was plain milk. The pre-exposed rats consumed an average of $0.85 \mathrm{~g} / \mathrm{kg} / \mathrm{hr}$ during the 0 -s delay condition and $1.10 \mathrm{~g} / \mathrm{kg} / \mathrm{hr}$ of ethanol during the 6 -s delay condition. The nonexposed rats consumed an average of 0.69 and $0.97 \mathrm{~g} / \mathrm{kg} / \mathrm{hr}$ for the 0 -s and 6-s delay conditions respectively. When the terminal reinforcer was ethanol milk the pre- 
exposed subjects consumed slightly less ethanol ( $3.63 \mathrm{~g} / \mathrm{kg} / \mathrm{hr}$ versus $3.88 \mathrm{~g} / \mathrm{kg} / \mathrm{hr}$ ) than the non-exposed subjects did during the 0-s delay condition when the terminal reinforcer was ethanol. The pre-exposed subjects consumed an almost identical amount of ethanol $(3.70 \mathrm{~g} / \mathrm{kg} / \mathrm{hr})$ when compared to the non-exposed subjects $(3.72$ $\mathrm{g} / \mathrm{kg} / \mathrm{hr}$ ) during the 6-s delay condition. A three way mixed ANOVA was conducted to further investigate if there was any effect of group on ethanol consumption. The between group measure was home cage exposure to ethanol (pre-exposed or nonexposed). The within group conditions were delay to terminal reinforcement delivery (0-s or 6-s); and, terminal reinforcer quality (plain milk or ethanol milk). No significant three-way interaction was found $(F(1,11)=0.88, \mathrm{NS})$ and there was no significant effect of delay $(\mathrm{F}(1,11)=3.01, \mathrm{NS})$. There was a statistically significant effect of terminal reinforcer quality $(F(1,11)=174.98, p<0.01)$. The data from the delay conditions were combined for each reinforcer quality enabling a two way ANOVA to be conducted. The between subject factors were again pre-exposed or non-exposed; and the within subject factor was terminal reinforcer quality (plain milk or ethanol milk). There was a significant effect of quality $(F(1,24)=332.12, p<0.001)$ but no interaction between quality and group $(\mathrm{F}(1,24)=0.84, \mathrm{NS})$. As no statistically significant between group differences in ethanol consumption were found the group data were combined into a single group for the remainder of the analyses.

\section{Preference}

When the data from Phase II and Phase III were plotted as a function of location (left versus right lever) of the $\mathrm{MN}$ contingency, it was apparent that all of the subjects had developed side biases (Figure 3.2). Using terminal reinforcer quality (plain sucrose, 


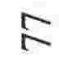

$\stackrel{\infty}{q}$
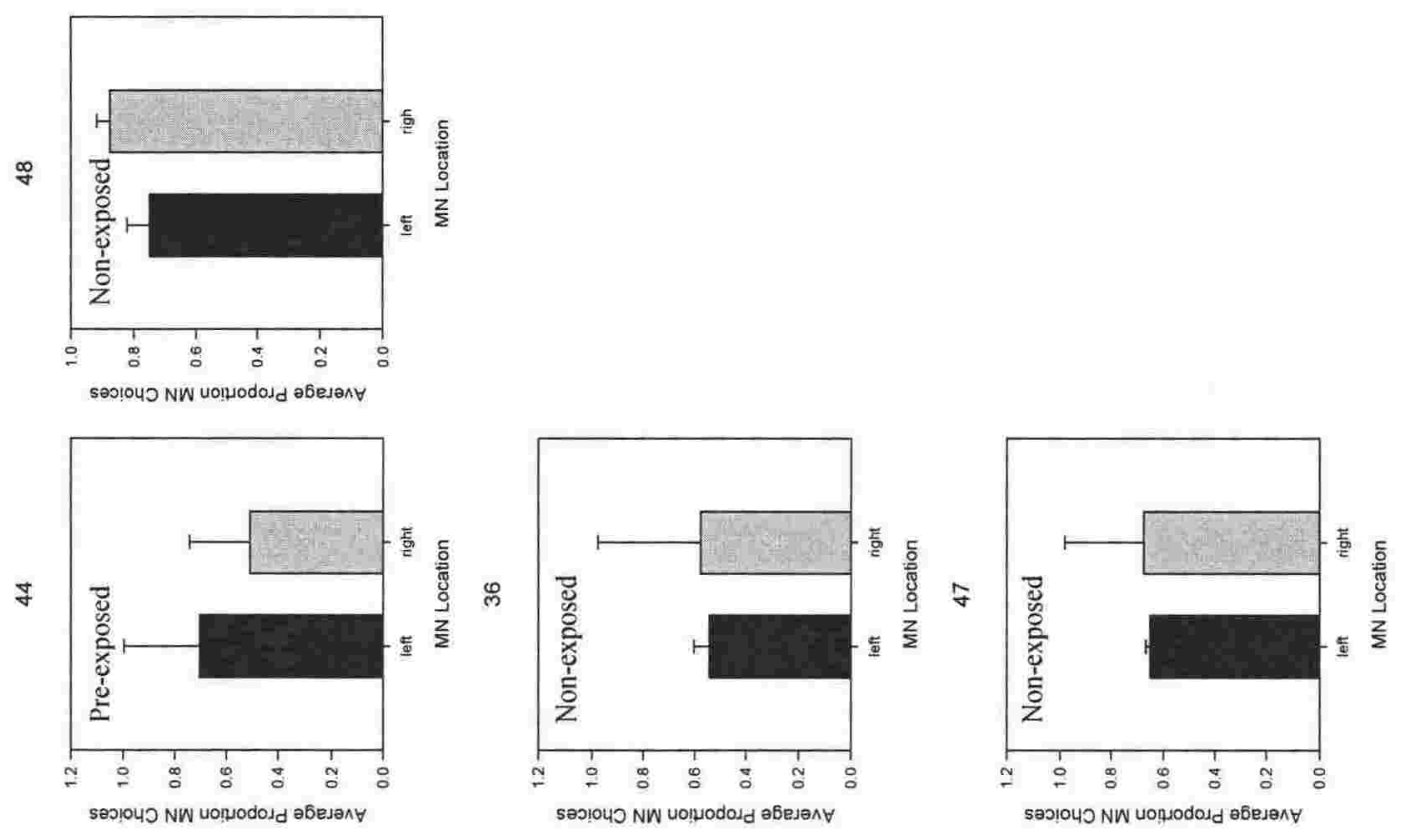

永

突离

矛总京

巳 吾

ב 5

$=z_{v}$

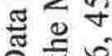

口

竞

ह

跴

ㄴ. $\frac{0}{0}$

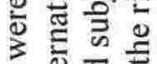

的总

흥흐 흘

言总方

匹

西

ब.

잉 च
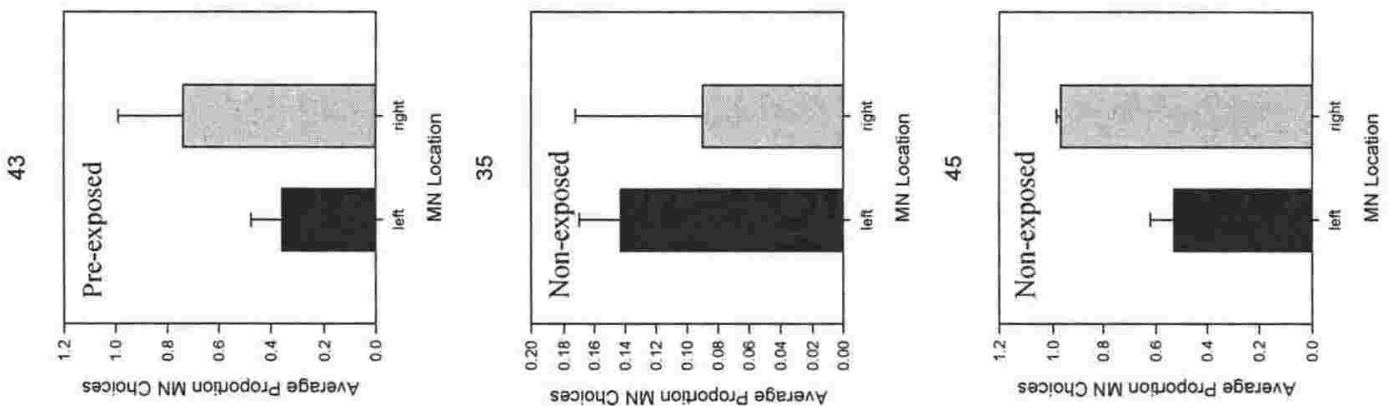

ฮ

mo

उ诺

거을

ษัํํㅇํㅇ

के छे

乙

टू $\sum$ ह

의

के ले ठํ

बํㅝㄹ
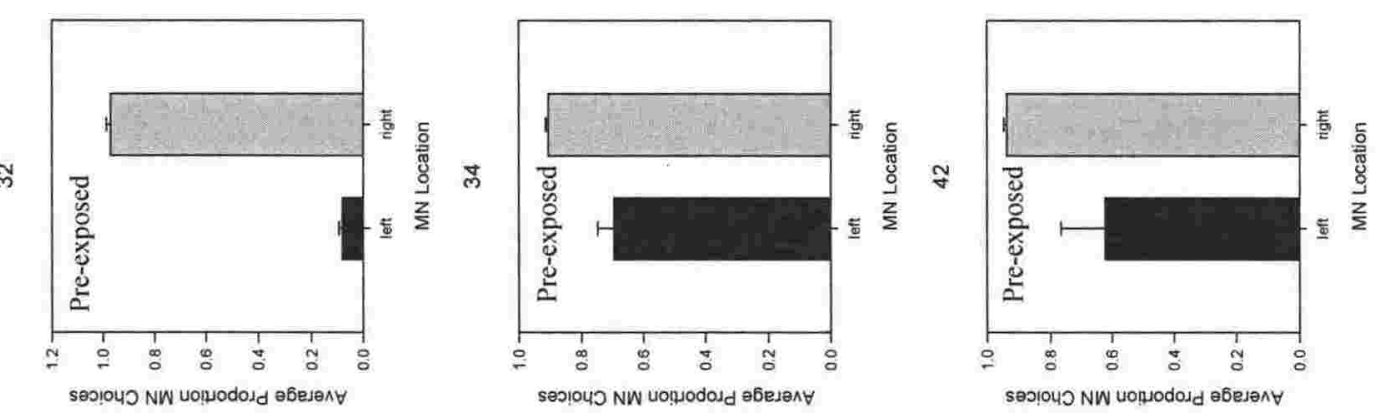

跑苍

悹里

ठิऐ

\%

ڤँ

过 究

흥

ป bै

$z$ E

记

을 을
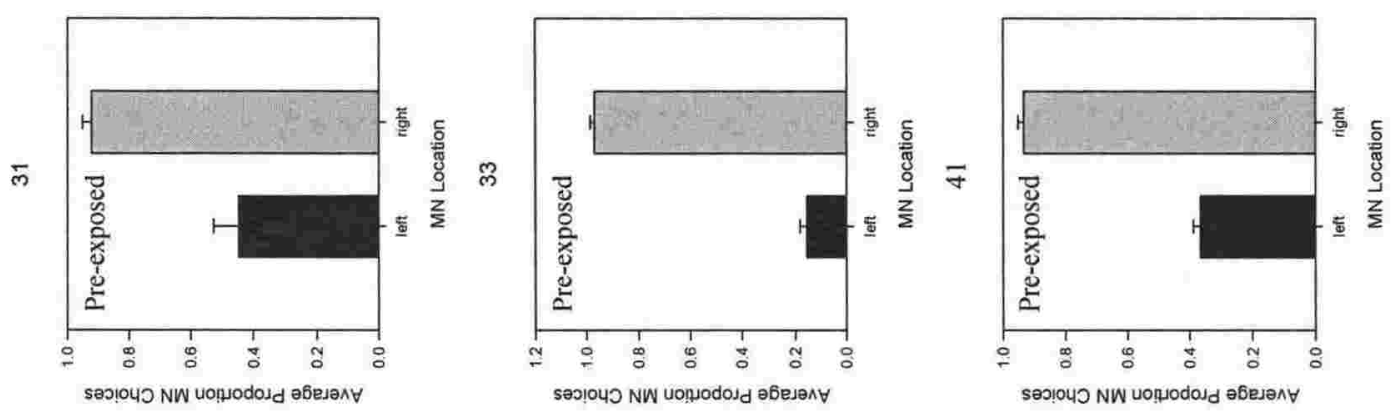

원 要

훙 क्ञ

品 을

㐘艺

文吉

i.

ก) 岕

는

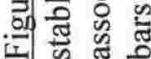


ethanol sucrose) and location of the $\mathrm{MN}$ contingency (left, right) a repeated measures ANOVA found a significant effect of both quality $(F(1,12)=98.23, p<0.001)$ and location $(\mathrm{F}(1,12)=22.41, \mathrm{p}<0.001)$ on responding. The bias found using the current $\mathrm{MN}-\mathrm{ML}$ paradigm is not without precedent. For example, in a concurrent chains paradigm, Rachlin and Green (1972) used forced choice trials in addition to free choice trials to reduce side bias. Two different approaches were used to reduce the effect of bias for data analysis.

\section{Proportion MN Choices}

The first approach to minimise the influence of side bias combined the average proportion of $\mathrm{MN}$ choices when the $\mathrm{MN}$ contingency was associated with the left and right levers for each delay condition (see Figure 3.3). When the terminal reinforcer was plain milk (Phase II) and the delay to the terminal reinforcers decreased from 6-s to $0-\mathrm{s}$, the proportion of $\mathrm{MN}$ choices decreased for eleven of the thirteen subjects. The remaining two subjects became slightly more impulsive (increased proportion of $\mathrm{MN}$ choices).

Changing the terminal reinforcer from plain milk to ethanol milk in Phase III resulted in a change in preference for all thirteen rats (Figure 3.3). All of the subjects increased their responding on the self-controlled alternative and chose the MN alternative less frequently at the 0 -s delay when the terminal reinforcer quality was changed. Increasing the delay to $6 \mathrm{~s}$ while keeping the terminal reinforcer quality the same (i.e. ethanol milk), did not have a systematic effect on choice behaviour. Nine of the subjects became more impulsive as the delay to ethanol milk terminal 
2
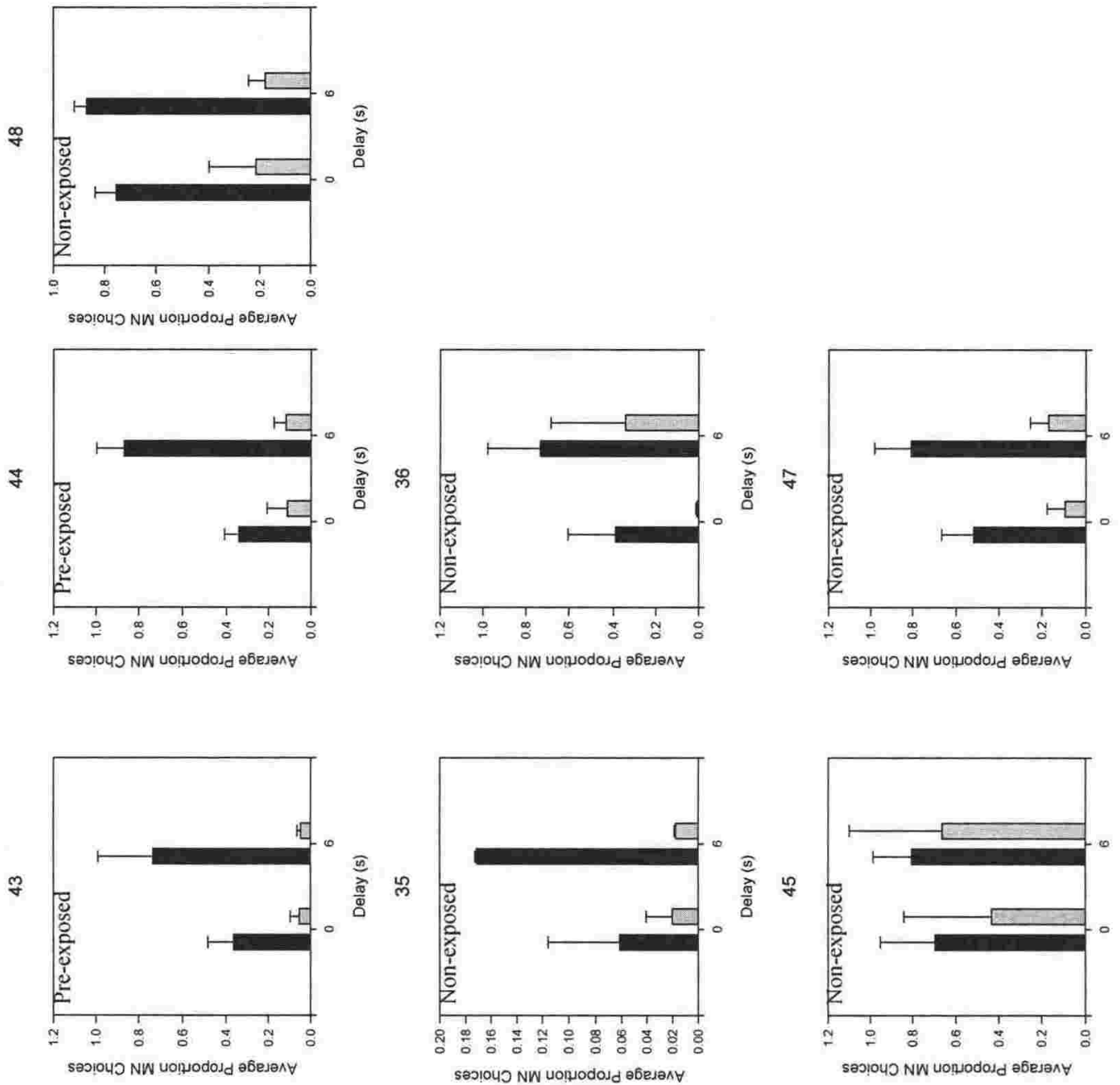

E

昰问

후응

들 跑

흔 융

망

ป⿱艹

福

을

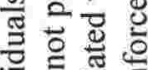

는

$\Xi$ 品

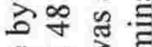

믈

Nㅜ응

ชิ ช.

的亨

에

zñ

ㅇㅠㅎㅀ

을 造

के है

०ूँ

엥

욜 त

훙ㅎㅎ

¿气

‥ ․ㅡㄹ

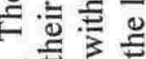

m. $\cong$ 를

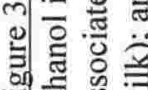
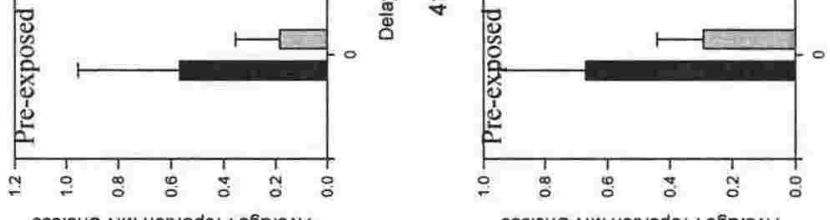
reinforcers increased. Two subjects were less impulsive and the remaining two subjects did not change their preference when the delay changed. A within subjects repeated measures ANOVA was conducted with reinforcer quality (plain milk versus ethanol milk) and delay to terminal reinforcer delivery (0-s versus 6-s) as factors. There was a significant effect of reinforcer quality $(F(1,12)=74.08, p<0.01)$ and delay $(\mathrm{F}(1,12)=19.04, \mathrm{p}<0.01)$ but no statistically significant interaction between quality and delay $(\mathrm{F}(1,12)=0.89, \mathrm{p}>0.05)$.

\section{$\log p$ and $\log$ bias}

Although the proportions measure revealed some patterns in the data, there are some limitations to this measure. A potentially better measure that has parallels to $\log d$ in the signal detection literature is $\log p$ (Equation 12) which has a related measure of bias. The data from this experiment was therefore re-analysed using log preference $(\log p) . \log p$ represents the geometric mean of $\mathrm{MN}$ versus $\mathrm{ML}$ responses independent of which side each contingency is on. Log $p$ partials out side bias in a manner similar to the $\log d$ equation used by (Davison \& Tustin, 1978; White $\&$ Alsop, 1993).

$$
\log p=0.5 \cdot \log \left[\left(\frac{M N_{L}}{M L_{R}}\right)\left(\frac{M N_{R}}{M L_{L}}\right)\right]
$$

For the $\log p$ equation, $\mathrm{MN}_{\mathrm{L}}$ and $\mathrm{MN}_{\mathrm{R}}$ are the choices of the More Now contingency when the impulsive contingency is associated with the left and right levers respectively. Likewise, $\mathrm{ML}_{\mathrm{R}}$ and $\mathrm{ML}_{\mathrm{L}}$ are the choices of the More Later contingency when the self-control contingency was located on the right and the left. A $\log p$ 
greater than 0 indicates that the subject prefers the impulsive $(\mathrm{MN})$ contingency; less than 0 shows preference for the self-controlled (ML) contingency, and equal to 0 shows the subject was indifferent to the choice contingencies. That is, as $\log p$ increases preference for the impulsive alternative increases.

The results of the $\log p$ analysis are shown in Figure 3.4. When the terminal reinforcer was plain milk and the delay to terminal link reinforcement increased preference for the $\mathrm{MN}$ alternative increased. Therefore, subjects were more impulsive at the 6-s delay than they were at the 0-s delay. In Phase III, the terminal reinforcer was changed to ethanol milk and increasing the delay to the terminal reinforcer from 0 $\mathrm{s}$ to $6 \mathrm{~s}$ did not have a consistent effect on preference in the initial link. Statistical analysis yielded results similar to those from the proportion of $\mathrm{MN}$ choices measure.

An equation similar to $\log p$ can be used to determine the extent and direction of bias (Equation 13). For log bias, a value greater than 0 is a bias for the left alternative regardless of location of the choice contingencies. Log bias less than 0 indicates a bias to the right alternative, and if $\log$ bias equals 0 there is no side bias.

$$
\log \text { bias }=0.5 \cdot \log \left[\left(\frac{M N_{L}}{M L_{R}}\right)\left(\frac{M L_{L}}{M N_{R}}\right)\right]
$$

All subjects that completed this study were biased, and bias changed as delay and quality changed (Figure 3.5). The parameters for $\log$ bias are as follows: $\mathrm{MN}_{\mathrm{L}}$ and $M N_{R}$ are choices of the impulsive alternative when it is located on the left and the right; and $M L_{L}$ and $M L_{R}$ are choices made on the self-controlled option when it is on 
న
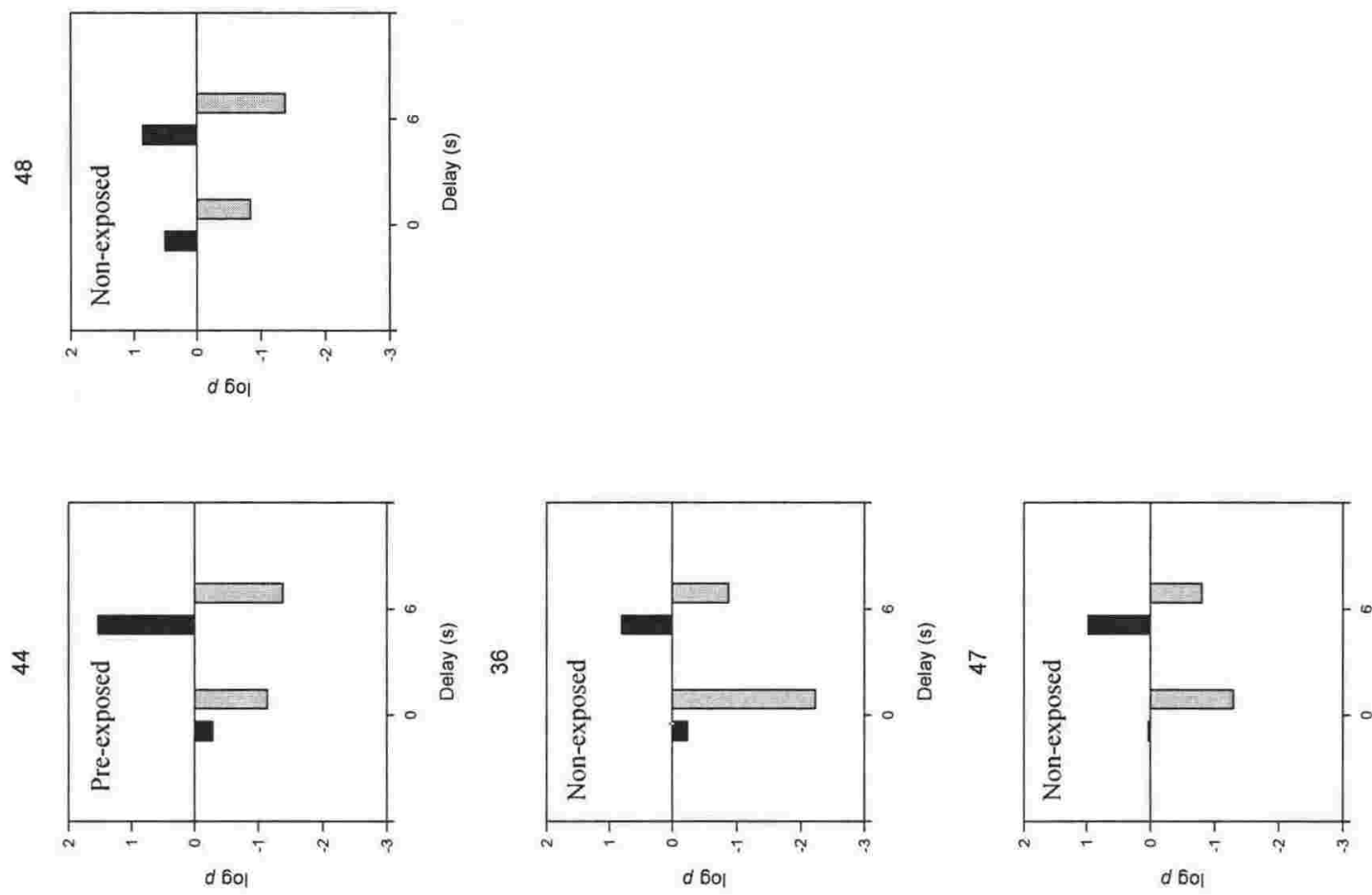

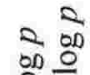

응

产

步

을

을

을

差 空

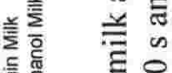

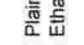

.

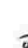

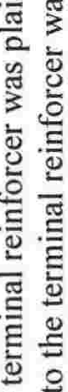

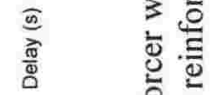

를
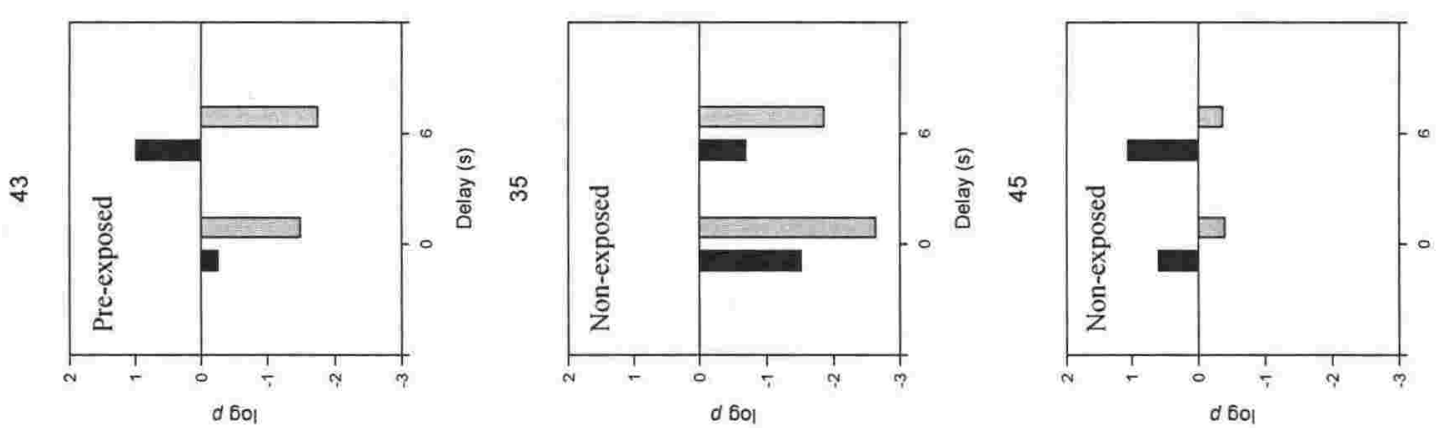

告
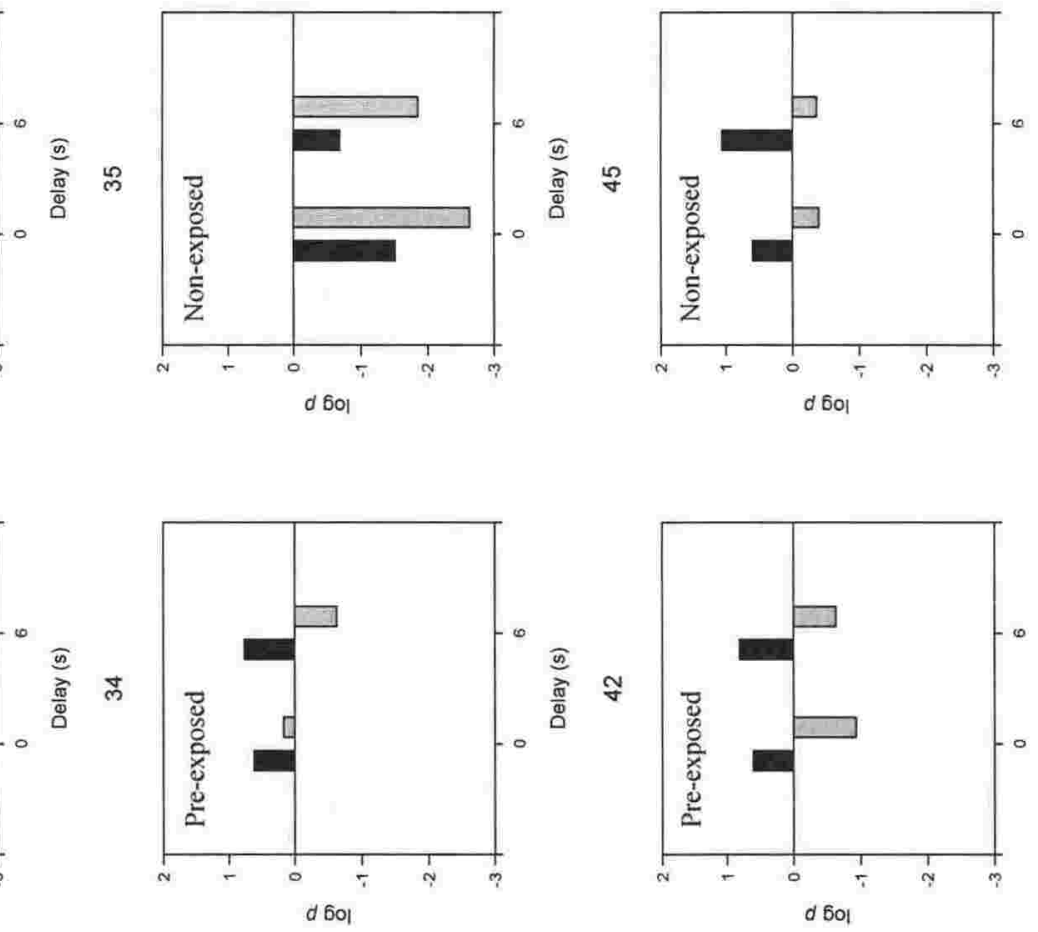

产

冚 $\frac{\overline{0}}{3}$

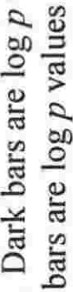

iี

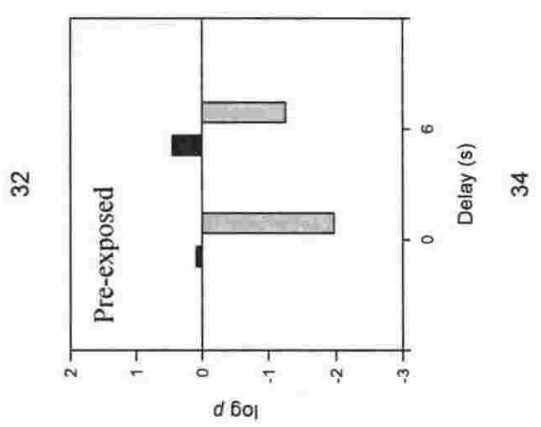

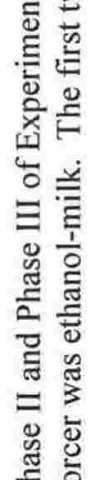

든

훙

证
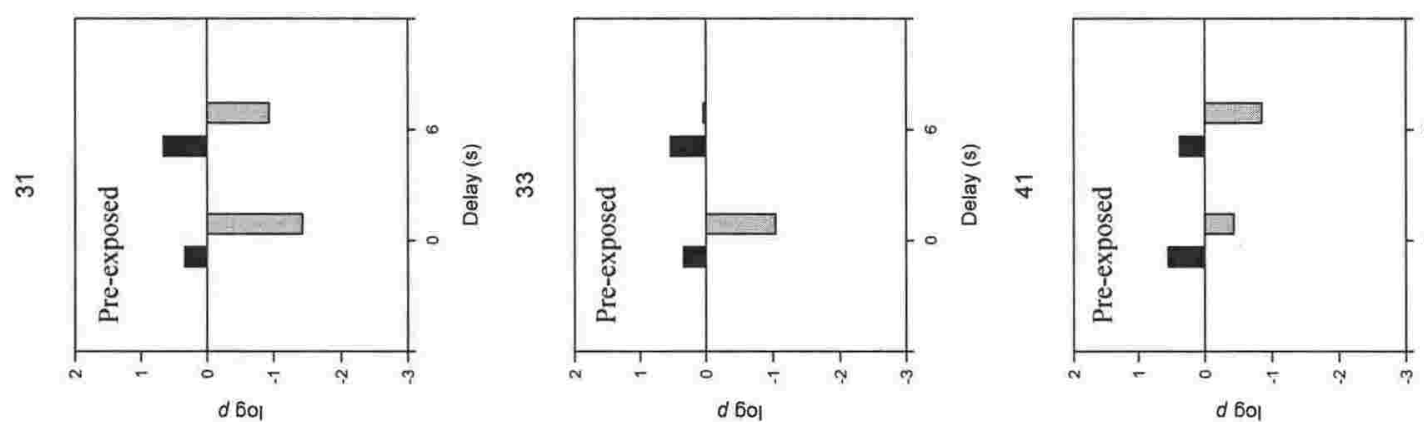

गे है

요ㅇㅛㅛ

ণं। $\frac{\bar{\Phi}}{3} \frac{\overline{0}}{3}$

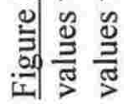


$\infty$
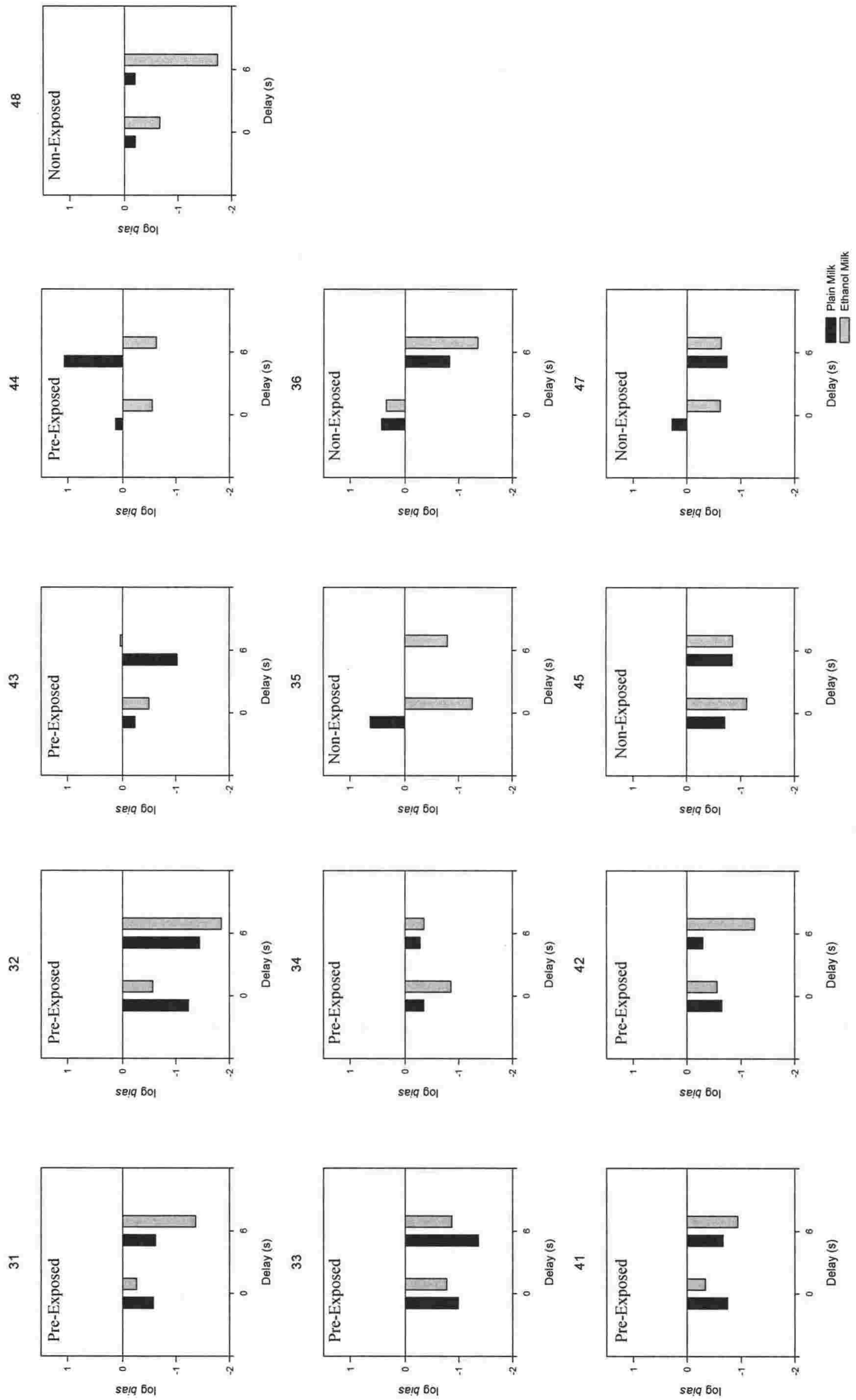

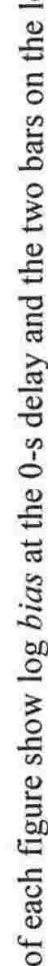


the left and the right of the operant chamber. All subjects were found to have bias, and bias varied unsystematically when delay and quality were manipulated.

\section{$\underline{\text { Discussion }}$}

The main finding for Experiment 2 was that both the initial and the terminal reinforcers influenced initial link behaviour distribution. Furthermore, the quality of the terminal link reinforcers influenced response distribution in the initial link. Contrary to the findings of Davison and Smith (1986), the addition of initial reinforcers did not eliminate sensitivity to the terminal link contingencies. As the absolute delay to the terminal reinforcers increased, and relative delay remained constant, preference for the $\mathrm{MN}$ reinforcer alternative increased. This occurred more systematically during Phase II when plain milk was the terminal reinforcer than during Phase III when subjects were reinforced with ethanol milk in the terminal links. This finding of an increase in preference for the impulsive alternative when the relative delay ratio remained $1: 1$ and absolute delay increased is not consistent with previous literature (Ito \& Asaki, 1982; Snyderman, 1983). However, it is consistent with the concept that the value of a reinforcer decreases as delay to delivery of that reinforcer increases (Grace, 1999; Grace \& Nevin, 1999; Mazur, 2001). As the delay to the terminal reinforcers increased in Phase II the value of those reinforcers decreased and the influence that the terminal link contingencies had on initial link behaviour decreased. Thus, the decreasing influence of the terminal link reinforcers meant that subjects were more likely to choose the $\mathrm{MN}$ initial link alternative, which delivered reinforcers of the preferred quality (ethanol milk).

The influence of terminal reinforcer quality on initial link behaviour was demonstrated in Phase III of the study when terminal reinforcer quality was changed 
from plain milk to ethanol milk. All thirteen subjects became significantly more selfcontrolled in Phase III and, with one exception, all preference measures calculated for that phase indicated that subjects were more self-controlled than they were in the previous phase. If subjects had disregarded reinforcer quality, behaviour would not have changed when the terminal reinforcer quality was changed.

The increase in self-control seen in all subjects when ethanol milk was substituted for plain milk clearly demonstrates that reinforcer quality is a factor that needs to be accounted for in models of self-control behaviour. Changing reinforcer quality could influence response distribution by changing sensitivity to delay or magnitude, or the effect could be multiplicative (as in the generalised concatenated matching law). The failure to find a statistically significant interaction between quality and delay in the current study suggests that quality manipulations did not change sensitivity to delay. The finding that there was not a statistically significant interaction between quality and delay was similar to the findings of Anderson and Woolverton (2003) who found no interaction between reinforcer concentration and delay to reinforcer delivery. In that study, drug dose was analogous to an amount manipulation and, when delay to reinforcer delivery was equal, subjects chose the larger dose most frequently. When an interaction is not found between two variables it indicates that the sensitivity parameters for one independent variable should not be affected by manipulations of a second independent variable, thus the current findings suggest that the sensitivity to delay parameters used in the GCML and CCM should not be affected by the quality manipulations. Alternatively, the influence of quality on response distribution may be captured as another independent variable $\left(\mathrm{Q}_{\mathrm{L}} / \mathrm{Q}_{\mathrm{R}}\right)$ in an extension of the concatenated 
explanation for the change in behaviour seen in Phase III is that the change occurred because adding ethanol to the terminal reinforcers increased the value of each terminal link by increasing the amount parameters by either a set value or a proportional value. That is, reinforcer quality may have a direct effect on reinforcer amount rather than on sensitivity to amount. Fitting the GCML to the data may have helped tease out these options but was not possible to collect enough data to do so.

Unfortunately, it was not possible to complete enough delay conditions to fit either the GCML or the CCM to the current data. This was because the experiment came to a premature end due to a supplier error. The supplier mistakenly delivered methanol rather than ethanol to the laboratory and, when the error was detected, the subjects from Experiment 2 were euthanised. Fortunately, the supplier error did not effect any of the data that is presented in this experiment. It did however impact on a third delay condition and no data from those conditions was included in the analysis. Thus, Experiment 3 was conducted to provide enough data for model fitting. The following experiment did not use the acclimatisation procedure that pre-exposed subjects to ethanol in their home cages before the operant experiments. This was because no statistically significant differences were found in the ethanol intake of the pre-exposed and the non-exposed groups in Experiment 2. 


\section{EXPERIMENT 3}

\section{Modification of the $M N-M L$ Procedure}

Experiment 3 was an extension of Experiment 2. The purpose of this experiment was to complete more delay conditions and examine quantitatively the influence of delay to terminal reinforcer delivery on preference using a MN - ML paradigm. A minimum of three delay conditions was required to fit quantitative models to the results.

The current study used ethanol as the terminal link reinforcer. This reinforcer was selected as the results of Experiment 2 indicated that, in general, rats responding on the MN-ML paradigm tended to respond most frequently on the lever associated with the impulsive contingency. The results of Experiment 2 indicate that increasing the delay to the terminal reinforcers increases impulsivity; possibly, by decreasing the influence the terminal link contingencies have on initial link behaviour. If the less valuable plain milk was used as the terminal reinforcer it is possible that increases in the delay could result in a ceiling effect beyond which further increases in delay would not change behaviour. That is, behaviour may become exclusively impulsive when the delay to the terminal reinforcers is relatively brief. However, the findings in Experiment 2 also suggested that ethanol delivery in the terminal link may increase self-control responding in the initial link which would minimise this potential problem.

For the current study, several procedural changes were made to the $\mathrm{MN}-\mathrm{ML}$ procedure used in Experiment 2. Firstly, concurrent fixed interval (FI) schedules 
were used in the initial links instead of concurrent FR 1 FR 1 schedules, which were used in Experiment 2. The purpose of the FI schedules was to enable more behavioural data to be collected, potentially increasing the reliability of the choice measure, and to ensure that the initial link durations remained equal. Secondly, the solution vehicle changed from diluted sweetened condensed milk to $10 \%$ sucrose. This change was made because much of the existing literature regarding ethanol as a reinforcer compares responding maintained by a sucrose solution to that maintained by ethanol sucrose solutions (e.g., Heyman \& Oldfather, 1992; Samson, Roehrs, \& Tolliver, 1982).

The final procedural change made for Experiment 3 was that this study used a modified version of the $\mathrm{MN}-\mathrm{ML}$ paradigm designed to reduce side bias. Using forced choice trials is a method commonly used to reduce side bias (Mazur, 1986; Rachlin \& Green, 1972). Forced choice trials ensure that subjects experience both of the reinforcement contingencies. The difference in the approaches used in the two cited studies is that one begins experimental sessions with several forced choice trials (Rachlin \& Green, 1972) and the other disperses the forced choice trials throughout a session (Mazur, 1986). Given that using forced choice trials at the beginning of the sessions did not eliminate bias in Experiment 2, the approach used in the current study was similar to Mazur (1986) in that forced choice trials and free choice trials alternated throughout the session. Sessions were divided into thirteen blocks of four trials each. Trial blocks began with two forced choice trials and ended with two free choice trials. It was predicted that this procedural manipulation would increase the salience of the locations of the $\mathrm{MN}$ and the $\mathrm{ML}$ contingencies and thereby decrease side bias. 
The main purpose of Experiment 3 was to collect data from enough delay conditions to enable quantitative model fitting. The model of interest was the GCML. The GCML predicts that behaviour will not change if the ratio of the delays to terminal link delivery are held constant when the absolute duration of the delays is increased. That is, because the GCML depends on relative rather than absolute delays to terminal reinforcement the model predicts that behaviour will not change as the delay to terminal link delivery increases. Although a shift in behaviour occurred when absolute delay to terminal reinforcer delivery increased in Experiment 2 problems with the procedure may have contributed to behavioural changes. Most notably, all of the subjects in Experiment 2 developed strong side biases. Thus, one aim of Experiment 3 was to modify the procedure to reduce bias and then determine if the GCML was able to describe the obtained data.

Method

\section{Subjects}

Six male Norway-hooded rats were subjects for Experiment 3. The rats were kept at $85 \%$ of their free-feeding body weight. Housing conditions were the same as for Experiment 1.

\section{Apparatus}

The apparatus used for Experiment 3 was the same apparatus used in Experiment 1. Each operant chamber had a houselight. There were two retractable levers on the front wall with a light above and a dipper below each lever. There was also a fixed lever on the back wall with a red light positioned above it. 


\section{Procedure}

\section{Reinforcement Contingencies}

The paradigm used was a More Now - More Later (MN - ML) procedure similar to the one used in Phase III of Experiment 2. The main procedural change was that each session was broken down into blocks of four trials. The first two trials of each block were forced choice trials. On those trials, the trial initiation response resulted in only one front lever being inserted into the chamber. There was always one forced MN trial and one forced ML trial per block of four. The order of forced choice trial presentation was random. Subjects had to respond to receive one $\mathrm{MN}$ forced choice reinforcer and one $\mathrm{ML}$ forced choice reinforcer before they were able to advance to the free choice trials. The final two trials of each block were free choice trials.

During the free-choice trials, both front levers were inserted into the chamber and subjects were able to respond on either lever.

Regardless of trial type, each $\mathrm{MN}$ response was reinforced with one dip of ethanol sucrose in the initial link and one dip of ethanol sucrose in the terminal link. ML responses were reinforced with a single dip of plain sucrose in the initial link and three dips of ethanol sucrose in the terminal link. The dipper located below the lever associated with the MN contingency delivered ethanol sucrose reinforcers and plain sucrose reinforcers were delivered by the dipper located below the lever associated with the ML contingency.

Three delays to terminal reinforcer delivery were studied: 5-, 20-, and 40-s. The order of presentation of the delay conditions was counter balanced (Table 4.1). Delay conditions were organised so that the MN contingency was initially associated with 


\section{Table 4.1}

Order of delay conditions for each subject in Experiment 3.

\begin{tabular}{|c|c|c|c|}
\hline Subject & Delay Condition & Left Contingency & Right Contingency \\
\hline \multirow[t]{6}{*}{ E11 } & $5 \mathrm{~s} \quad(18)$ & $\mathrm{MN}$ & ML \\
\hline & $5 \mathrm{~s} \quad(14)$ & ML & $\mathrm{MN}$ \\
\hline & $20 \mathrm{~s}(14)$ & ML & $\mathrm{MN}$ \\
\hline & $20 \mathrm{~s}(14)$ & $\mathrm{MN}$ & ML \\
\hline & $40 \mathrm{~s}(14)$ & $\mathrm{MN}$ & ML \\
\hline & $40 \mathrm{~s}(14)$ & ML & $\mathrm{MN}$ \\
\hline \multirow[t]{6}{*}{ E21 } & $20 \mathrm{~s}(18)$ & $\mathrm{MN}$ & ML \\
\hline & $20 \mathrm{~s}(16)$ & ML & $\mathrm{MN}$ \\
\hline & $40 \mathrm{~s}(16)$ & ML & $\mathrm{MN}$ \\
\hline & $40 \mathrm{~s}(14)$ & $\mathrm{MN}$ & ML \\
\hline & $5 \mathrm{~s} \quad(14)$ & $\mathrm{MN}$ & ML \\
\hline & $5 \mathrm{~s} \quad$ (15) & ML & $\mathrm{MN}$ \\
\hline \multirow[t]{6}{*}{ E31 } & $40 \mathrm{~s}(18)$ & $\mathrm{MN}$ & ML \\
\hline & $40 \mathrm{~s}(14)$ & ML & $\mathrm{MN}$ \\
\hline & $20 \mathrm{~s}(14)$ & ML & $\mathrm{MN}$ \\
\hline & $20 \mathrm{~s}(14)$ & $\mathrm{MN}$ & ML \\
\hline & $5 \mathrm{~s} \quad(14)$ & $\mathrm{MN}$ & ML \\
\hline & $5 \mathrm{~s} \quad$ (14) & ML & $\mathrm{MN}$ \\
\hline \multirow[t]{6}{*}{ E41 } & $40 \mathrm{~s}(18)$ & $\mathrm{MN}$ & $\mathrm{ML}$ \\
\hline & $40 \mathrm{~s}(15)$ & ML & $\mathrm{MN}$ \\
\hline & $5 \mathrm{~s} \quad$ (14) & ML & $\mathrm{MN}$ \\
\hline & $5 \mathrm{~s} \quad(14)$ & $\mathrm{MN}$ & ML \\
\hline & $20 \mathrm{~s}(14)$ & $\mathrm{MN}$ & ML \\
\hline & $20 \mathrm{~s}(15)$ & ML & $\mathrm{MN}$ \\
\hline \multirow[t]{6}{*}{ E51 } & $5 \mathrm{~s} \quad(20)$ & $\mathrm{MN}$ & ML \\
\hline & $5 \mathrm{~s} \quad(24)$ & ML & $\mathrm{MN}$ \\
\hline & $40 \mathrm{~s}(14)$ & ML & $\mathrm{MN}$ \\
\hline & $40 \mathrm{~s}(14)$ & $\mathrm{MN}$ & ML \\
\hline & $20 \mathrm{~s}(14)$ & $\mathrm{MN}$ & ML \\
\hline & $20 \mathrm{~s}(22)$ & ML & $\mathrm{MN}$ \\
\hline \multirow[t]{6}{*}{ E61 } & $5 \mathrm{~s} \quad(18)$ & $\mathrm{MN}$ & ML \\
\hline & $5 \mathrm{~s} \quad$ (14) & ML & $\mathrm{MN}$ \\
\hline & $40 \mathrm{~s}(14)$ & ML & $\mathrm{MN}$ \\
\hline & $40 \mathrm{~s}(16)$ & $\mathrm{MN}$ & ML \\
\hline & $20 \mathrm{~s}(14)$ & $\mathrm{MN}$ & ML \\
\hline & $20 \mathrm{~s}(14)$ & ML & $\mathrm{MN}$ \\
\hline
\end{tabular}

Note: Numbers in brackets indicate sessions to reach stability. 
the left lever and the ML contingency was associated with the right lever then, once responding had met the stability criteria, the positions of the $\mathrm{MN}-\mathrm{ML}$ contingencies were reversed. Only the free choice trials were used to determine stability. The stability criteria were that the five session moving average of the proportion of responses on the $\mathrm{MN}$ alternative of the most recent sessions differed by no more than 0.05 from the previous five session average for two consecutive blocks; and that there was no monotonically increasing or decreasing trend in the session data. Sessions lasted 110 minutes or until 13 blocks of trials were completed, whichever occurred first.

\section{MN-ML Procedure}

All sessions began with the front levers retracted and all lights off. If the $\mathrm{MN}$ contingency was associated with the left lever the houselight remained off but if the MN contingency was associated with the right lever the houselight was turned on. Trial start was signalled by illumination of the back light. For forced choice trials, the trial initiating response turned off the back light and one of the front levers was inserted into the chamber and its associated light was turned on. An FI 10-s schedule began timing from when the front lever was inserted into the chamber. When the schedule was complete, a further response by the subject retracted the front lever and the initial reinforcer was delivered. The delay to terminal reinforcer delivery began counting down as soon as the final initial link response was made. The front light associated with the active lever remained on during the delay. When the delay had elapsed, the front lever was again inserted into the chamber. A response (FR 1) on the lever retracted the lever, turned off the light, and delivered either one dip of ethanol sucrose for MN trials or three dips of ethanol sucrose for ML trials. Each dip of reinforcer was available for $4 \mathrm{~s}$. A 60-s ITI (blackout) separated the trials and, if the 
session was a B condition, the houselight was turned off during the blackout. If a subject failed to respond to complete a forced choice trial within 10 s of the FI elapsing the lever was retracted and the front light was extinguished. A blackout commenced which was equal in duration to the amount of time required to complete a trial. At the end of the blackout the forced choice trial was repeated. That is, subjects could not proceed to the free choice trials until one MN and one ML forced choice trial was completed.

Two free choice trials followed completion of the forced choice trials. Free choice trials were similar to forced choice trials excepting that the initiating back lever response was followed by both of the front lights being turned on and the two front levers being inserted into the chamber. Independent FI 10-s FI 10-s schedules began timing immediately following the back lever response. The subjects were free to distribute their responses between the two front levers. A 1-s changeover delay (COD) limited switching between the levers. The first response made after the FI had elapsed was the choice response. The choice response determined both initial link reinforcer quality and the terminal link contingency. For example, if the $\mathrm{MN}$ alternative was associated with the left lever a choice response on the left lever was an impulsive choice. A choice of the MN alternative turned off the right light and retracted both front levers. The light above the chosen (left) lever remained on and the initial link reinforcer was delivered. The delay to the terminal reinforcer began timing from the choice response and the light associated with the initial link choice remained on during the delay. After the delay had elapsed, the light above the front lever went off and the light above the back lever was turned on. A response (FR 1) on the back lever extinguished the back light and was immediately followed by delivery 
of the appropriate amount of terminal reinforcement (one dip of ethanol sucrose for the current example) from the dipper located in the front wall of the chamber. To ensure that MN and ML trial durations were equal a post-reinforcer blackout followed MN trials. Again, trials were separated by a 60-s ITI. Once the ITI elapsed, the light above the back lever was again illuminated and a response on the back lever initiated the next trial.

Results and Discussion

Contingency Control

After the subjects had completed all three delay conditions ( $5 \mathrm{~s}, 20 \mathrm{~s}$, and $40 \mathrm{~s}$ ), data from the last five sessions of each contingency condition were used to determine if there was contingency control. It was concluded that there was contingency control if there was no significant difference in the proportion of $\mathrm{MN}$ responses made when the location of the MN alternative alternated between the left and right lever. The six graphs in Figure 4.1 show the proportion of responses made on the lever associated with the MN contingency when the MN contingency was located on the left, and when it was located on the right. Single sample t-tests demonstrated that there was a significant difference in the proportion of $\mathrm{MN}$ responses made when the location of the $\mathrm{MN}$ contingency changed for one rat only $(\mathrm{t}(2)=4.96, \mathrm{p}<0.05)$. This indicates that the majority of subjects in this study did not have significant side biases, thus indicating that the procedural manipulation used in this study was largely effective in reducing bias. Due to the lack of significant side biases for most rats, the descriptive measures $\log p$ and $\log$ bias were not calculated for this experiment. 
E11

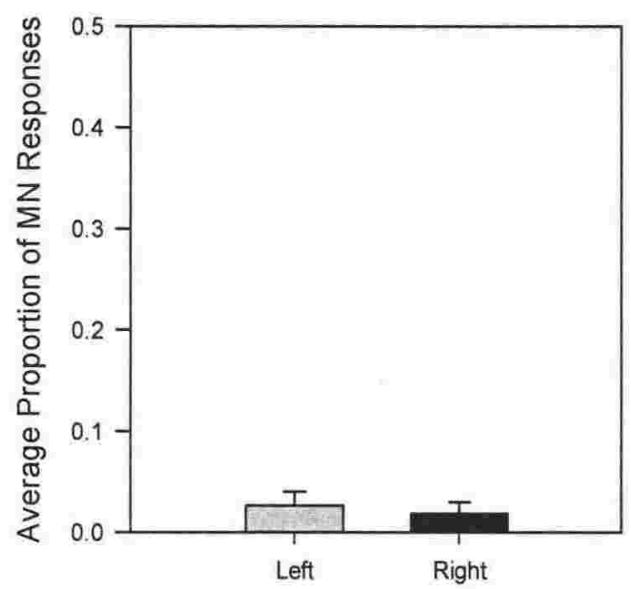

Location of MN Contingency

E31

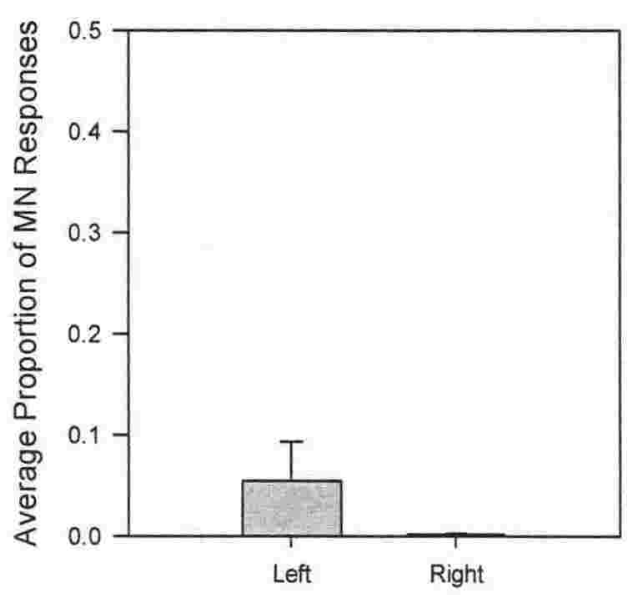

Location of MN Contingency

E51

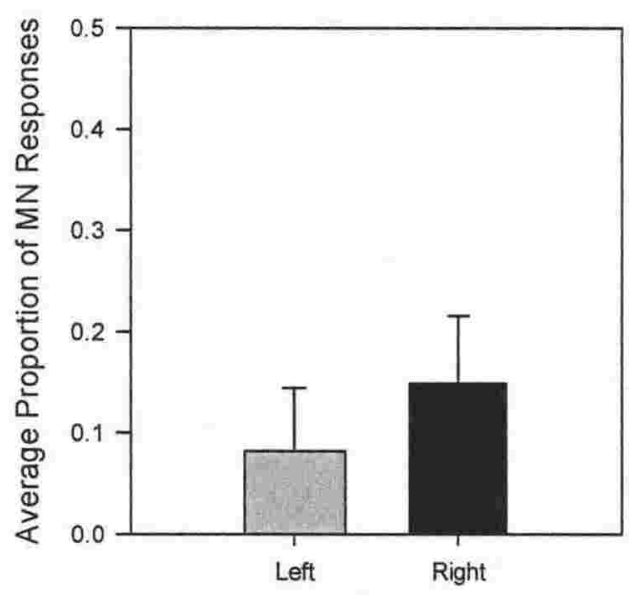

Location of MN Contingency
E21

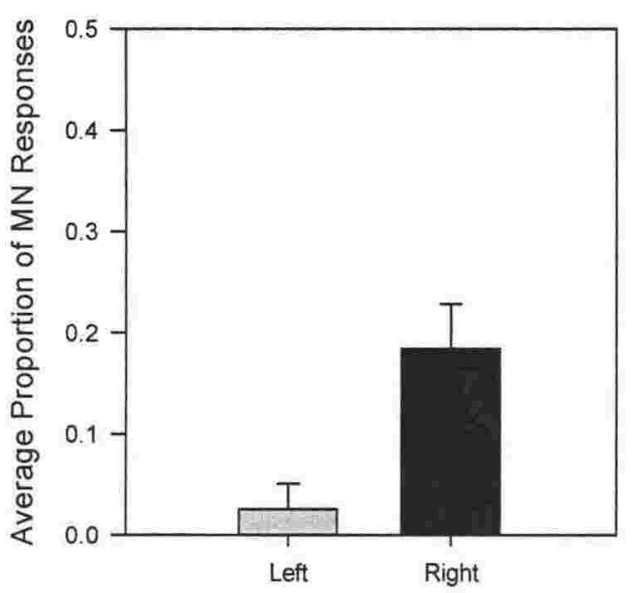

Location of MN Contingency

E41

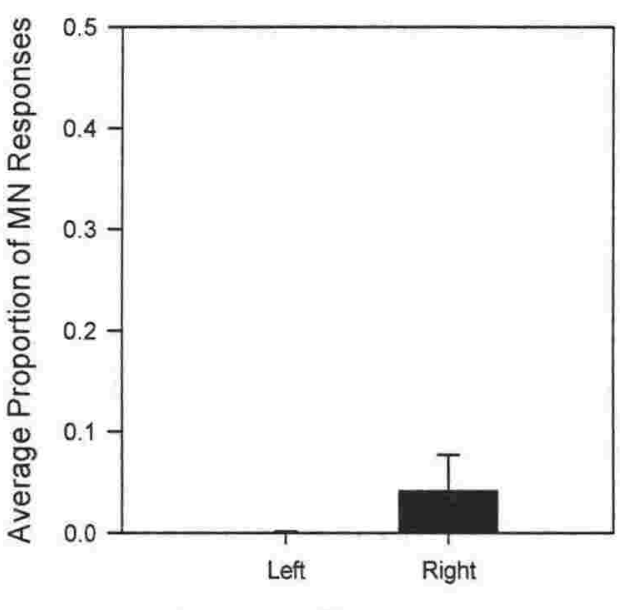

Location of MN Contingency

E61

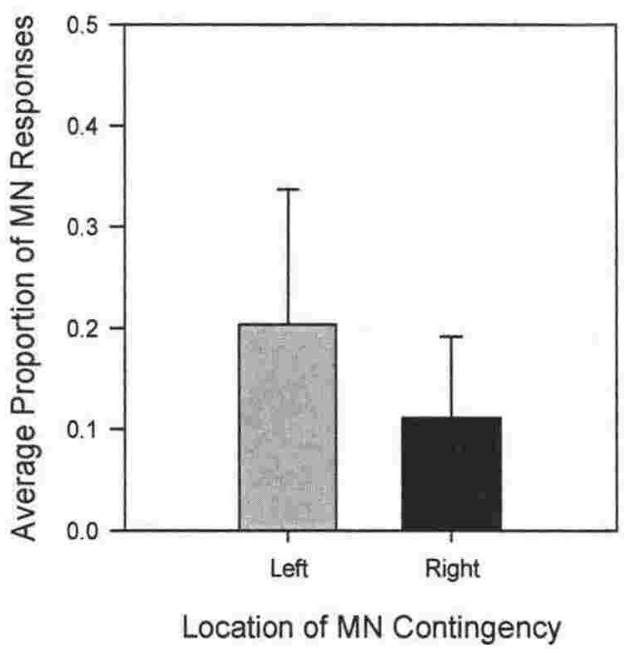

Figure 4.1. Contingency control graphs for each subject. The graphs show the proportion of $\mathrm{MN}$ responses made averaged over the last five sessions of each condition, the data from the three delay conditions was combined. The grey bars (Condition A) are the proportion of MN responses when the MN contingency was associated with the left lever. The black bars (Condition B) are proportions of $\mathrm{MN}$ responses when the $\mathrm{MN}$ condition was associated with the right lever. 


\section{Ethanol Consumption}

Data used to calculate ethanol consumption were the average number of $\mathrm{MN}$ and ML free and forced choices completed at each contingency condition (Table 4.2). The amount of ethanol subjects consumed decreased as the delay to terminal reinforcer delivery increased. Subjects consumed an average of $0.96 \mathrm{~g} / \mathrm{kg} / \mathrm{hr}$ during the 5 -s delay conditions, $0.86 \mathrm{~g} / \mathrm{kg} / \mathrm{hr}$ during the 20 -s delay conditions, and $0.75 \mathrm{~g} / \mathrm{kg} / \mathrm{hr}$ during the 40-s delay conditions. A repeated measures ANOVA with location of MN contingency (left, right) and delay to reinforcer delivery $(5 \mathrm{~s}, 20 \mathrm{~s}, 40 \mathrm{~s})$ revealed no significant effect on ethanol consumption of contingency location $(F(1,5)=0.958, N S)$ or interaction between location and delay $(\mathrm{F}(2,10)=1.24$, NS). There was a significant effect of delay $(\mathrm{F} 2,10)=10.02, \mathrm{p}<0.05$. As the delay to the terminal reinforcers increased, behaviour tended to become more impulsive and this meant that the amount of ethanol consumed by subjects decreased with increasing delay.

\section{Table 4.2}

Individual ethanol consumption $(\mathrm{g} / \mathrm{kg} / \mathrm{hr})$ for each delay condition

\begin{tabular}{cccc}
\hline Subject & $5 \mathrm{~s}$ & $20 \mathrm{~s}$ & $40 \mathrm{~s}$ \\
\hline E11 & 0.78 & 0.81 & 0.71 \\
E21 & 1.16 & 0.94 & 0.84 \\
E31 & 0.74 & 0.74 & 0.65 \\
E41 & 0.70 & 0.65 & 0.61 \\
E51 & 1.22 & 0.94 & 0.85 \\
E61 & 1.16 & 1.05 & 0.85 \\
\hline
\end{tabular}

\section{$\underline{\text { GCML Analysis }}$}

\section{Proportion MN Responses}

In this experiment the ratio of terminal link delays to reinforcement was always $1: 1$ and the absolute duration of the delay increased between conditions. According to the GCML this manipulation should not change the distribution of behaviour across the 
reinforcement alternatives. To assess this, the proportion of $\mathrm{MN}$ responses was averaged across conditions when the MN contingency was associated with the left lever and when it was associated with the right lever for each delay. The mean proportion of responses to the MN contingency was then plotted as a function of reinforcer delay (Figure 4.2). Contrary to predictions based on the GCML, the proportion of $\mathrm{MN}$ choices increased for five of the six subjects as the delay to delivery of the terminal reinforcer increased. The exception was Subject E11, who was not sensitive to the experimental contingencies and made approximately the same average proportion of $\mathrm{MN}$ responses at each delay. Figure 4.2, which shows the average proportion of $\mathrm{MN}$ responses made at each delay, clearly shows a shift in behaviour as a function of delay. Thus, the GCML was not fit to the data obtained in this study.

Unlike the CGML, the CCM (Equation 11) does describe behaviour that changes when the absolute delay to terminal reinforcer delivery increases. This is because increasing the delay to the terminal link reinforcers also increased the duration of the terminal links. Increases in terminal link duration increase the value of the temporal context exponent $\left(\mathrm{T}_{t} / \mathrm{T}_{\mathrm{i}}\right)$ and therefore behaviour should become more sensitive to the terminal link contingencies as absolute delay to reinforcer delivery increases.

\section{CCM}

An assumption of the CCM is that behaviour will change when the absolute delay to delivery of the terminal reinforcers changes. This is because changing the delay increases the duration of the terminal links; and, as the ratio of terminal- to initiallinks changes the impact of the terminal reinforcer amounts on initial link behaviour also changes. To visualise how increasing the delay to terminal reinforcer delivery 
E11

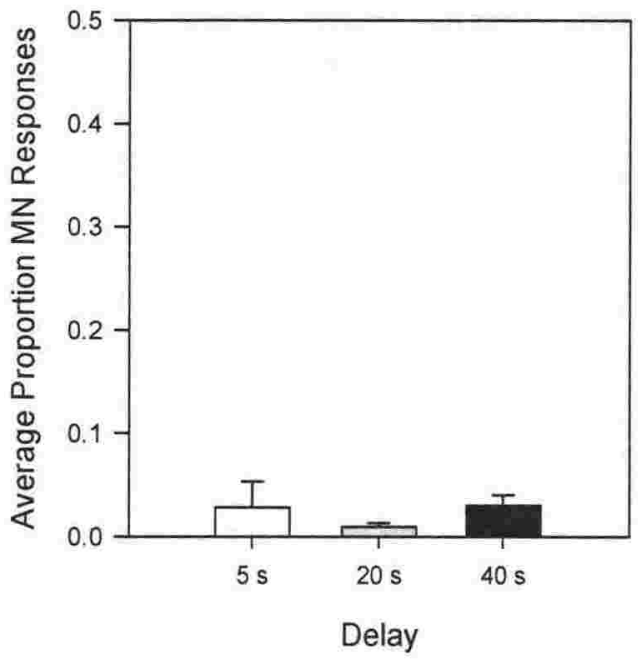

E31

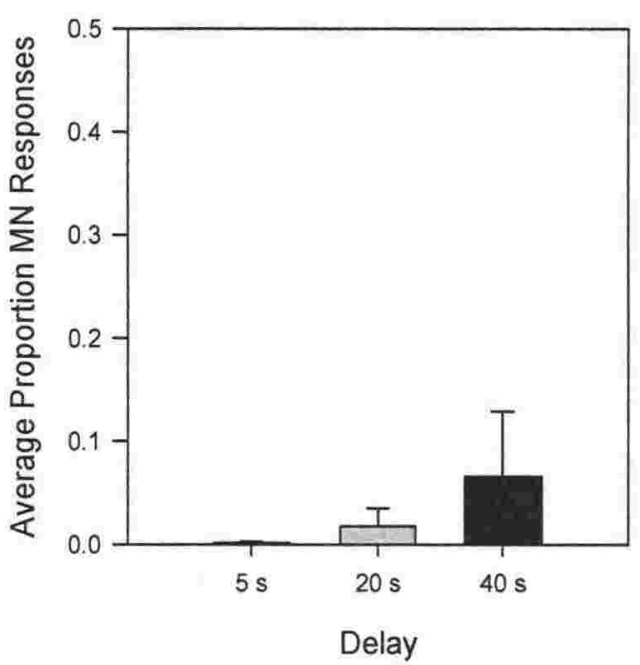

E51

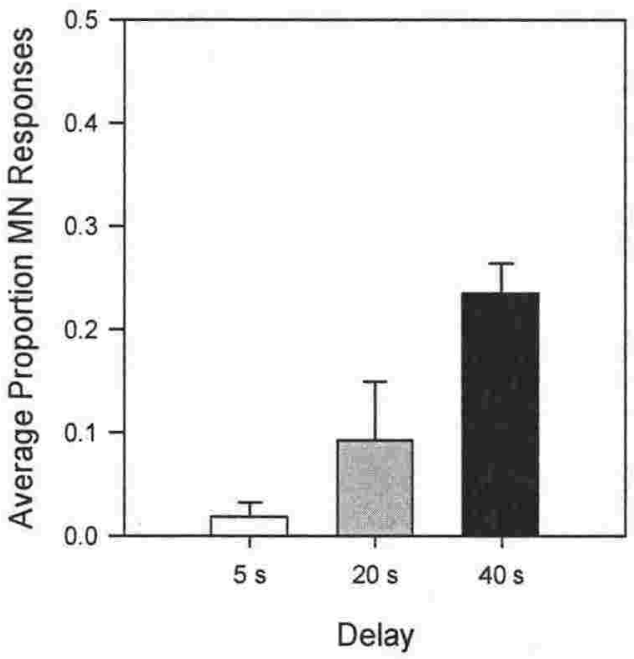

E21

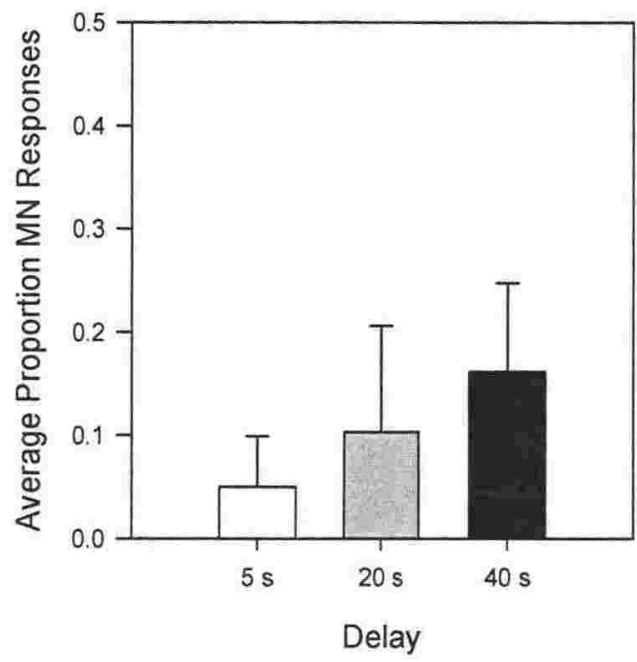

E41

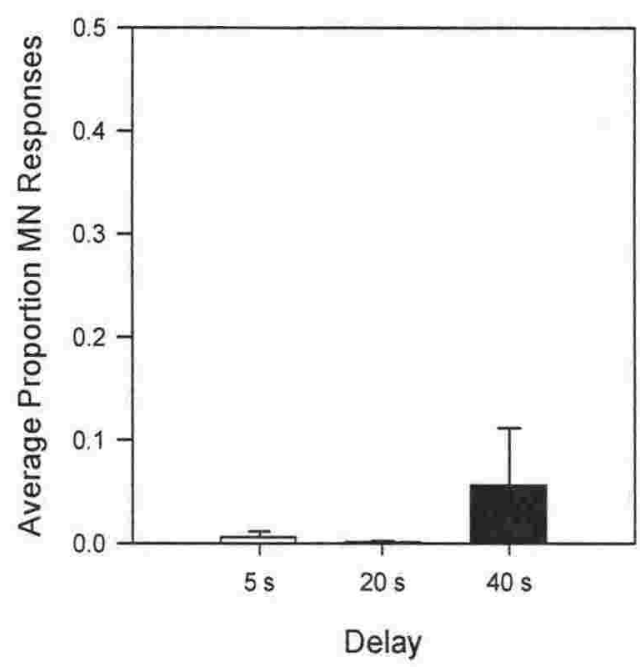

E61

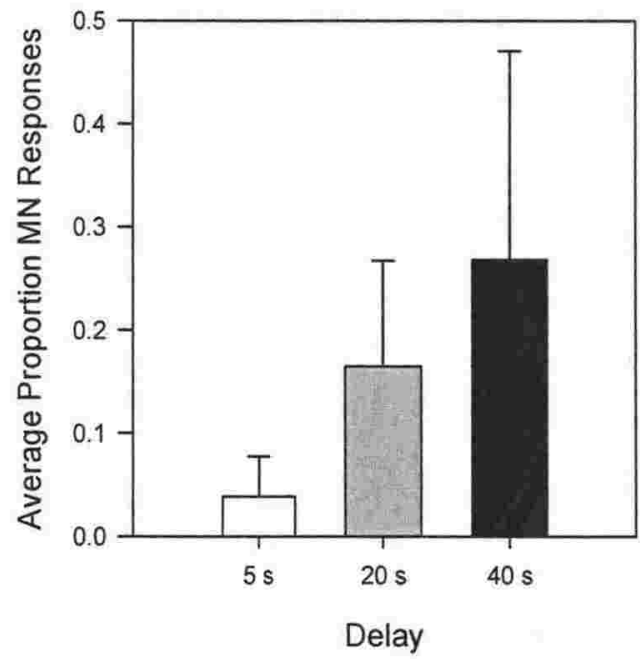

Figure 4.2. The average proportion MN responses made by each subject for each delay condition. The first bar of each set is for 5-s Delay conditions, the second bar is for the 20-s Delay conditions, and the third bar is for the 40 -s Delay conditions. Impulsivity increases as the proportion of MN choices increases. 
contingencies effected behaviour the data are presented in a series of graphs where the $\log$ obtained behaviour ratio is plotted as a function of the log amount ratio for each delay condition (the unfilled circles in Figure 4.3). The solid lines in Figure 4.3 provide a point of reference for the plotted data and show how behaviour would be distributed as a function of reinforcer amount in the absence of the moderating effects of the temporal context parameter or the free parameters. The data depicted in Figure 4.3 suggest that, firstly, the slope of the dashed line connecting the two data points in each graph is steeper than the solid reference line which indicates that behaviour tends to be representative of overmatching. Secondly, as the duration of the terminal links increases the slope of the dashed line tends to decrease (approach matching) for four of the six subjects. The slope of the line represents how sensitive a subject's behaviour is to the amount ratio. From Figure 4.3 it appears that increasing the delay to terminal reinforcer delivery decreases sensitivity to reinforcer amount. This can be seen across the three graphs provided for each subject as a decrease in the slope of the line as delay to terminal reinforcer delivery increases. The extent of the decrease in slope is not consistent between subjects.

In order to assess if the CCM model was able to adequately describe the data, it was necessary to modify the CCM to accommodate the inclusion of primary reinforcer delivery in the initial link. The assumption was made that the independent variables that comprise the initial link reinforcer contingencies combine multiplicatively in a 
E11

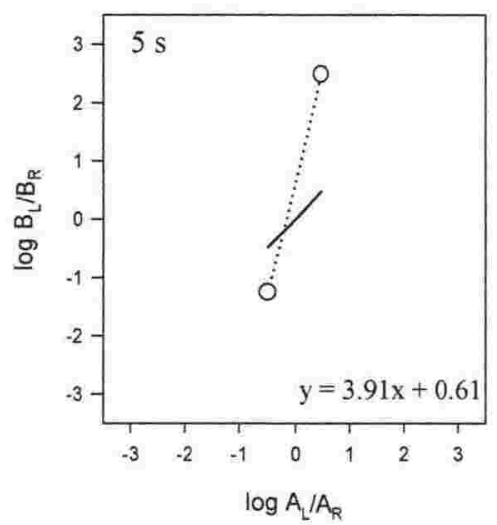

E21
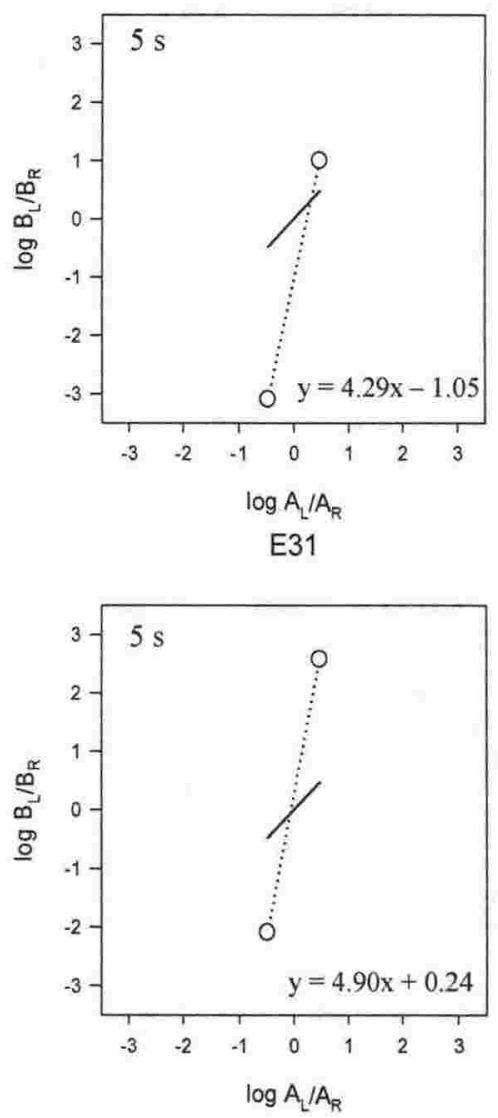

E11

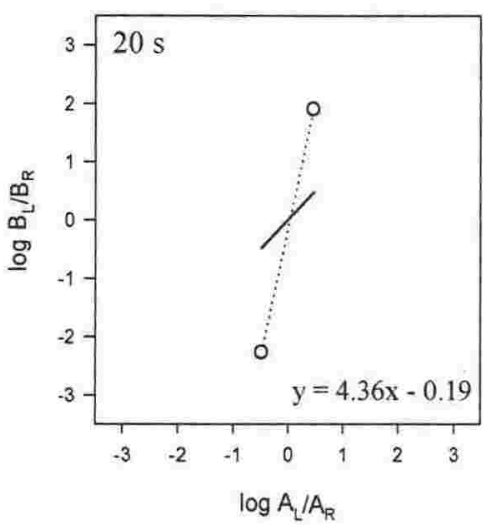

E21

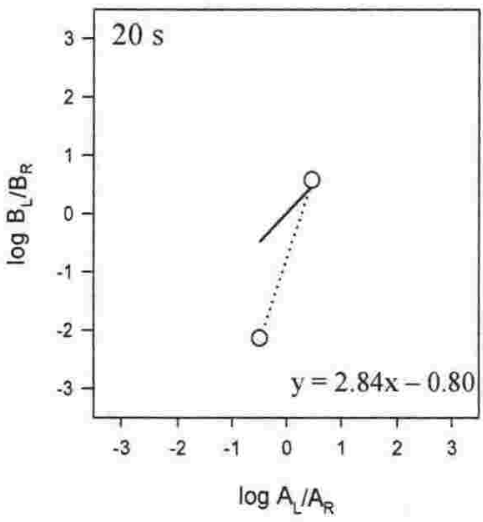

E31

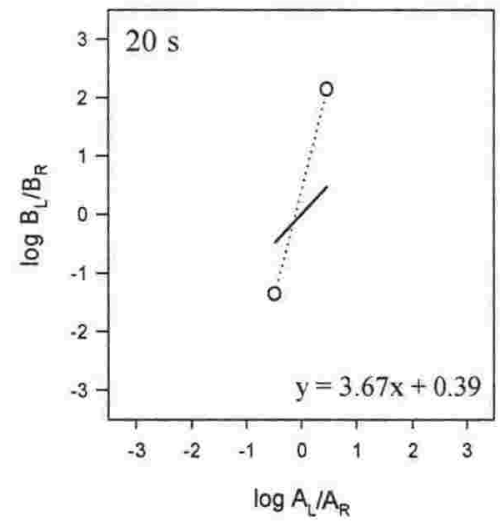

E11

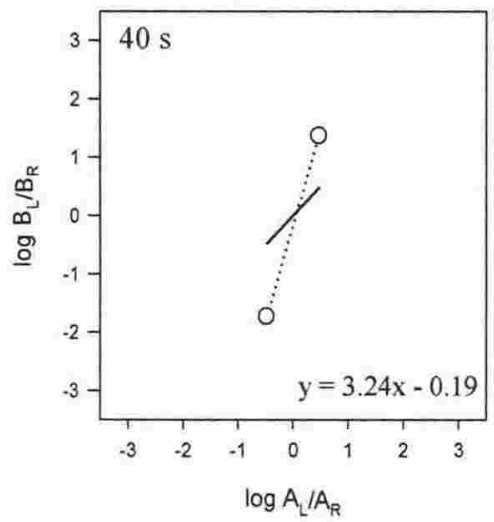

E21
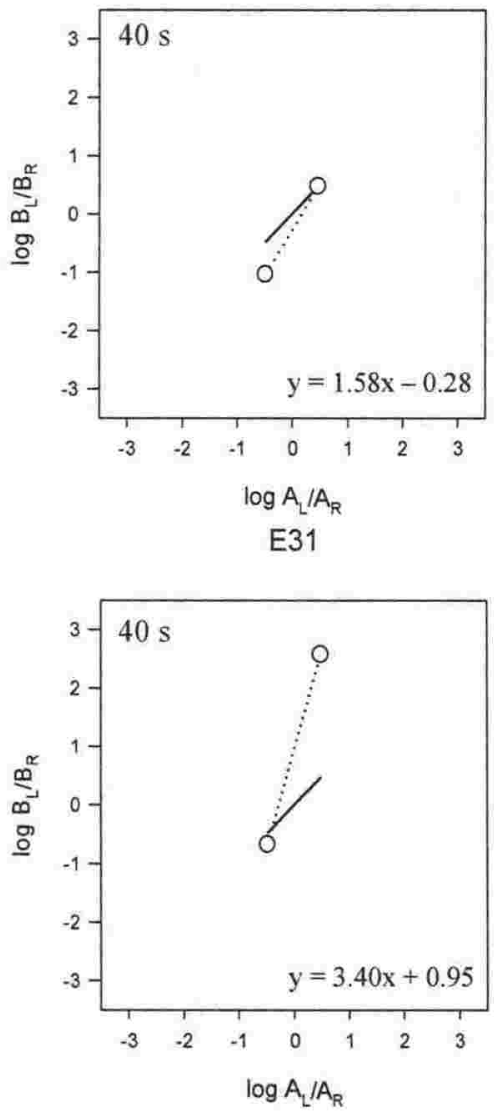

Figure 4.3. Individual subject graphs in which the log response ratios are plotted as a function of the log of the amount ratios for each delay condition of Experiment 3. The solid line on each graph represents strict matching of the behaviour ratio to the terminal link amount ratio. The dotted line is the obtained log behaviour ratio. Values are plotted for conditions in which the $\mathrm{MN}$ contingency was located on the left and also for conditions when it was located on the right. There are three graphs for each subject, one for each delay. 
E41

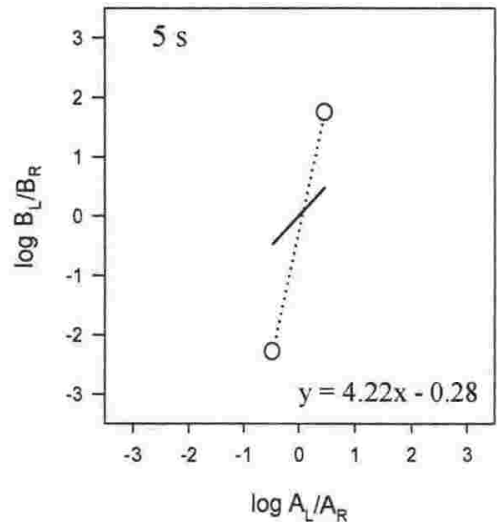

E51

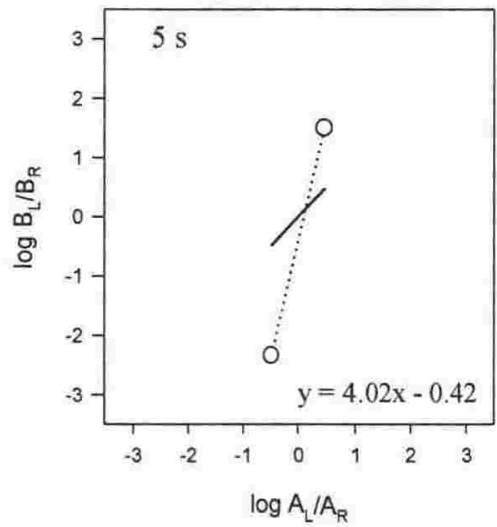

E61

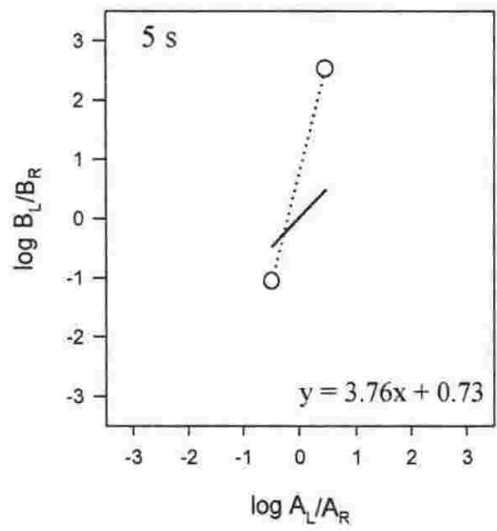

E41

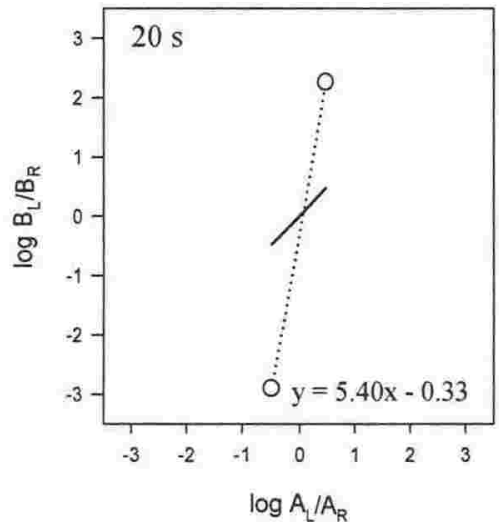

E51

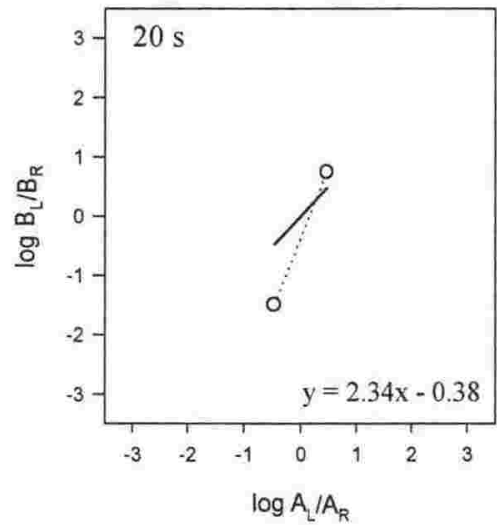

E61

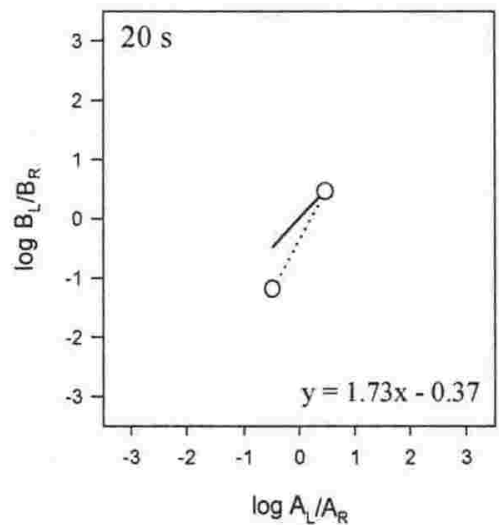

E41

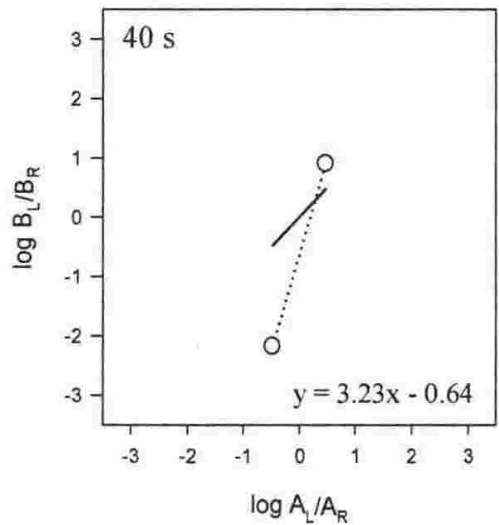

E51

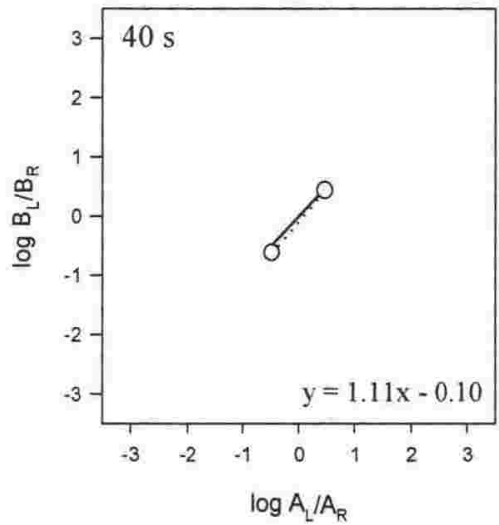

E61

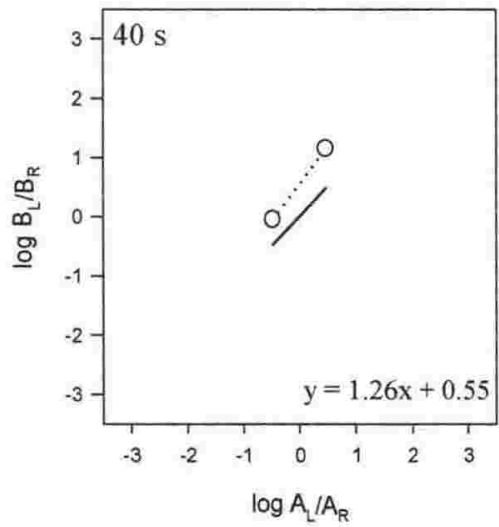

Figure 4.3. continued 
manner similar to the terminal link reinforcer variables (Equation 14).

$$
\frac{B_{L}}{B_{R}}=b\left[\left(\frac{R_{i L}}{R_{i R}}\right)^{a_{i R}}\left(\frac{A_{i L}}{A_{i R}}\right)^{a_{A}}\right]\left[\left(\frac{D_{t R}}{D_{t L}}\right)^{a_{t D}}\left(\frac{A_{t L}}{A_{t R}}\right)^{a_{t A}}\right]^{\left(\frac{T_{t}}{T_{i}}\right)^{k}}
$$

The modified CCM parameters are: $\mathrm{B}$ is the initial link behaviour allocated to a given alternative, $\mathrm{A}$ is reinforcer amount, $\mathrm{D}$ is delay to terminal reinforcer delivery, and $b$ is bias. The subscripts ${ }_{L}$ and $\mathrm{R}_{\mathrm{R}}$ indicate that the variable is associated with either the left or the right contingency, and the subscripts ${ }_{\mathrm{i}}$ and ${ }_{\mathrm{t}}$ denote the initial or the terminal link respectively. The addition of primary reinforcers in the initial link means that the ratio of the initial link reinforcer rates $(\mathrm{R})$ can be substituted for the terminal link entry rates in Equation 11 . The free parameters are: sensitivity to initial link reinforcer rate $\left(a_{R}\right)$, sensitivity to reinforcer amount $\left(a_{A}\right)$, and sensitivity to reinforcer delay $\left(a_{D}\right)$. Again, the subscripts ${ }_{i}$ and $_{t}$ denote whether the sensitivity parameter is an initial or terminal link parameter. As is usual for CCM, the temporal context ratio $T_{t} / T_{i}$ is the average duration of the terminal link divided by the average duration of the initial link; and $k$ is a scaling parameter. Several of the ratios in Equation 14 reduce to one for the current data set, including: the initial-link amount ratio, the initial-link reinforcer rate ratio, and the terminal-link delay ratio. To simplify the model it was assumed that reinforcer quality did not impact behaviour in the same way as other independent variables such as amount or delay. The modified CCM therefore reduces to Equation 15. 
$\frac{B_{L}}{B_{R}}=b\left[\left(\frac{A_{t L}}{A_{t R}}\right)^{a_{A}}\right]^{\left(\frac{T_{t}}{T_{i}}\right)^{k}}$

Equation 15 was first fit to the data with the scaling parameter set equal to one and the values of the bias parameter and sensitivity to the amount ratio were allowed to vary. Parameter values were determined using Microsoft Excel Solver. The results from this data fit are shown in Table 4.3 and it can be seen that CCM was able to account for a large percentage of the variance in the data. Examination of the data in Table 4.3 reveals that the subjects that had the steepest slopes in Figure 4.3 (E11, E31, and E41) were also the most sensitive to the amount ratio and were relatively selfcontrolled. The subjects that had lower sensitivity to the amount ratio (E21, E51, and E61) had shallower slopes and were the most impulsive.

\section{Table 4.3}

Derived values for bias (b) and sensitivity to the amount ratio $\left(a_{A}\right)$ when Equation 15 was fit to the data without including the scaling parameter $(k)$

\begin{tabular}{ccccc}
\hline Subject & $\mathrm{b}$ & $\mathrm{a}_{\mathrm{A}}$ & \% VAC & Avg Prop MN \\
\hline \hline E11 & 3.39 & 1.87 & 99.84 & 0.02 \\
E21 & 0.56 & 0.97 & 88.42 & 0.11 \\
E31 & 0.33 & 3.19 & 97.56 & 0.04 \\
E41 & 0.14 & 2.72 & 99.10 & 0.02 \\
E51 & 0.64 & 1.53 & 85.82 & 0.11 \\
E61 & 1.09 & 0.70 & 66.08 & 0.16 \\
\hline
\end{tabular}

As a comparison, data reported by White and Pipe (1987) have been reanalysed to determine if the current finding that increasing the delay decreased sensitivity to the reinforcer amount ratio was also found in their data. The White and Pipe study is similar to the current study in that the initial link schedules were concurrent FI FI and the delay ratio was held constant at 1:1 as the absolute duration of the delays 
increased. They studied three different amount ratios. A monotonic change in the slope of the line fit to each pair of delay/amount ratio was found in only seven of the fourteen cases (Table 4.4). For six of those cases, the slopes of the lines increased as the absolute delay to terminal reinforcer delivery increased. That is, increasing the delay to terminal reinforcer delivery increased sensitivity to reinforcer amount, which is the opposite to the results of the current study.

\section{Table 4.4}

Obtained slopes for each delay ratio from the re-analysis of White and Pipe's (1987) Experiment 2 when the log response ratio was plotted as a function of the log amount ratio for each delay

\begin{tabular}{ccccc}
\hline Subject & Amount Ratio & $0 \mathrm{~s}$ & $5 \mathrm{~s}$ & $20 \mathrm{~s}$ \\
\hline $\mathrm{G} 1$ & $2: 4$ & 0.08 & 1.30 & 1.63 \\
& $1: 5$ & 0.49 & 3.21 & 2.68 \\
& $1.5: 4.5$ & 0.36 & 2.30 & 2.49 \\
$\mathrm{G} 2$ & $2: 4$ & & & \\
& $1: 5$ & 0.55 & 2.54 & 1.34 \\
& $1.5: 4.5$ & 0.60 & 2.58 & 2.36 \\
$\mathrm{G} 3$ & & & 1.19 & 0.99 \\
& $2: 4$ & 0.31 & & \\
$\mathrm{G} 4$ & $1: 5$ & 1.38 & 3.22 & 2.30 \\
& & & & 2.68 \\
& $2: 4$ & 1.04 & 1.99 & 1.67 \\
& $1: 5$ & 2.55 & 2.49 & 2.31 \\
$\mathrm{G} 5$ & $1.5: 4.5$ & 1.80 & 1.77 & 1.85 \\
& & & & \\
& $2: 4$ & 0.33 & 0.68 & 1.35 \\
& $1: 5$ & 0.89 & 1.63 & 1.66 \\
& $1.5: 4.5$ & 0.20 & 1.40 & 2.05 \\
\hline
\end{tabular}

The major difference between procedure used in the current experiment and White and Pipe's study is the delivery of primary reinforcers in the initial link of the $\mathrm{MN}-$ ML procedure. Grace (1994) suggested that it is possible that procedural variations will moderate the effect of temporal context. In the current study, it is possible that the inclusion of primary reinforcement in the initial links changed temporal context, 
which resulted in a different pattern of behaviour change in the current experiment as compared to the White and Pipe study. For example, the effect of the different reinforcer qualities may have increased the discriminability of the two alternatives. Grace (1994) suggested that the effect of such procedural variations on behaviour might be accounted for by the CCM if the scaling parameter $(k)$ is allowed to vary. Equation 15 was used to calculate hypothetical changes in the proportion of behaviour allocated to the alternative associated with the smaller terminal reinforcer amount as the duration of the terminal link increases relative to the duration of the initial link (Figure 4.4). To generate the predictions, the amount ratio was set 1:3, bias and sensitivity to the amount ratio were set to one, and the value of $k$ was varied from negative one to positive one. The average terminal and initial link durations used in this study were used to obtain hypothetical predictions of behaviour change as delay to reinforcer delivery increases. As the terminal link duration increases (and the initial link duration remains constant) the value of the contextual parameter increases.

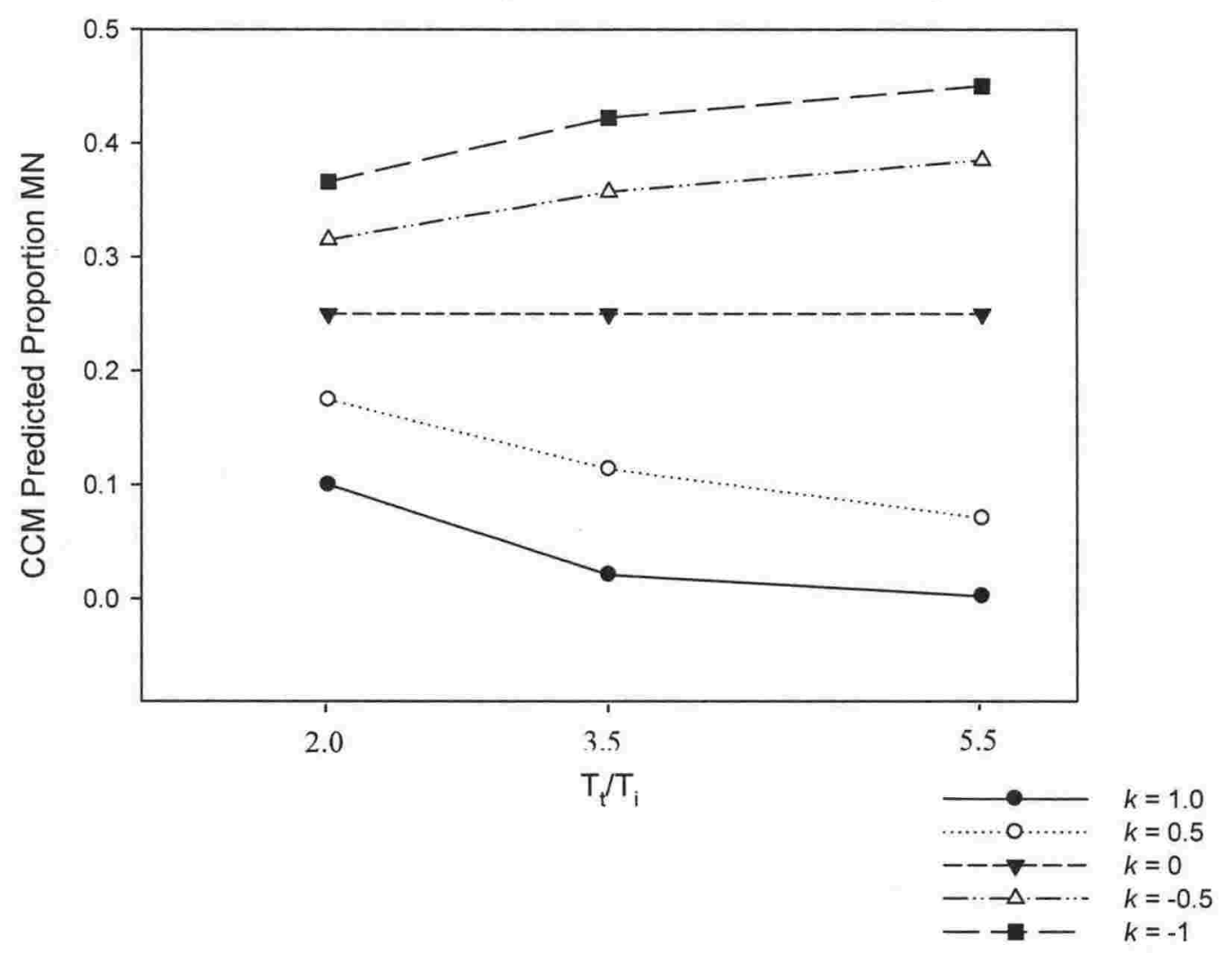

Figure 4.4. Choice proportions predicted by the CCM for several different scaling parameter values for Experiment 3 contingencies. The proportion of $\mathrm{MN}$ responses is predicted to decrease as the duration of the terminal link increases when $\mathrm{k}$ is a positive number. When $\mathrm{k}$ is negative increasing the duration of the terminal link is predicted to increase the proportion of responses allocated to the $\mathrm{MN}$ contingency. 
The impact of increasing the temporal context parameter on the apparent sensitivity of behaviour allocation to the ratio of reinforcer amounts will depend on how sensitive per se behaviour is to temporal context. The scaling parameter $k$ can be interpreted as being a measure of how sensitive behaviour is to temporal context. If a procedural variation decreases the impact of temporal context on behaviour this would be reflected in a negative $k$ value. In the context of the current experiment, a negative $k$ value would mean that as the difference between the terminal and initial links increased the value of the temporal context parameter would decrease. The effect of this is to reduce the impact of the terminal link reinforcer ratio on initial link responding. If the terminal reinforcer does not control behaviour, behaviour would tend to indifference or impulsivity (if ethanol is favoured).

If a procedural variation increases the impact of temporal context on behaviour the value of $k$ would be positive. A positive $k$ value would mean that the impact of temporal context on behaviour allocation would increase as the difference between the terminal and initial link durations increased. For the current experiment, an increase in the value of temporal context would mean that the impact of the terminal links on initial link behaviour would become more extreme as delay to the terminal links increased. This would be reflected in a shift towards the reinforcer alternative that delivers the larger amount of reinforcer (behaviour would become more selfcontrolled). Thus, by including the scaling parameter, the CCM is able to describe data from procedures in which impulsivity increases as delay to reinforcer delivery increases (e.g. the current study); and, when impulsivity decreases as delay increases (Ito \& Asaki, 1982; Snyderman, 1983; White \& Pipe, 1987). 
The model fit was repeated using the CCM and the three free parameters (bias, sensitivity to reinforcer amount, and the scaling parameter) were allowed to vary. The starting values for bias and sensitivity to reinforcer amount were set to one, and the starting value for $k$ was set to negative one. This was because the average proportion of behaviour allocated to the $\mathrm{MN}$ alternative by five of the six subjects increased as the delay increased. This increase in impulsivity is described by the CCM if the scaling parameter $(k)$ is negative (Figure 4.4). The results of the model fitting are shown in Table 4.5. The derived values of $k$ were negative for the five subjects that shifted their behaviour towards the impulsive alternative as the delay to delivery of the terminal reinforcers increased. Thus, as the delay to delivery of the terminal reinforcers increased sensitivity to the temporal context decreased which in turn decreased sensitivity to the terminal reinforcer amount ratio. This overall decrease in sensitivity to amount is reflected in a shift in behaviour in the direction of impulsivity (or indifference), which appears in Figure 4.3 as a shift towards matching as delay increases.

$\underline{\text { Table } 4.5}$

Free parameter values derived from fitting Equation 15 to the data from Experiment 3. when the temporal context scaling parameter $(k)$ was allowed to vary

\begin{tabular}{cccccc}
\hline & $\mathrm{b}$ & $\mathrm{a}_{\mathrm{A}}$ & $k$ & \% VAC & Prop MN \\
\hline E11 & 1.44 & 2.82 & 0.19 & 99.86 & 0.02 \\
E21 & 0.40 & 3.96 & -0.48 & 99.63 & 0.11 \\
E31 & 5.49 & 13.46 & -0.89 & 100.00 & 0.04 \\
E41 & 0.09 & 35.26 & -1.26 & 99.98 & 0.02 \\
E51 & 0.72 & 9.12 & -1.22 & 99.67 & 0.11 \\
E61 & 2.62 & 45.25 & -2.21 & 91.73 & 0.16 \\
\hline
\end{tabular}

Since procedural variations are captured by the scaling parameter, the next step is to consider the differences between the current study which saw a decrease in sensitivity as delay increased with the procedure used by White and Pipe (1987) which resulted 
in an increase in sensitivity as delay increased. The most obvious difference between the two procedures is the delivery of primary reinforcers in the initial link of the MN - ML paradigm. It thus appears that the different findings in the two studies are due to delivery of primary reinforcement in the initial links. The initial link reinforcers are delayed equally and are of equal amounts. The difference between the initial link reinforcers is their quality. Equation 15 does not have a parameter included that is specifically designated to account for the impact of reinforcer quality on behaviour. Thus, it appears that the impact of the initial link reinforcers has been captured in the current analysis by the inclusion of the temporal context scaling parameter. It could be that temporal context was affected by the addition of initial link reinforcers or by the quality of those reinforcers. The current study was not designed to differentiate between these two possibilities. However, if the different qualities of the initial link reinforcers reduced the impact of temporal context on behaviour the effect of the different qualities might show up as a preference for one of the qualities.

An effect of a decreased sensitivity to the terminal reinforcer amount ratios is that the control of initial link behaviour by terminal link contingencies also decreases. A decrease in control by the terminal link logically means that control of behaviour by the initial link contingencies would increase. As far as the initial link reinforcers are concerned, if behaviour is not influenced by reinforcer quality subjects should eventually become indifferent to the two reinforcer alternatives. If, however, subjects preferred ethanol sucrose to plain sucrose it would be likely that subjects would respond more frequently on the alternative that delivered ethanol sucrose as the delay to the terminal reinforcers increased. This change in response allocation would appear as an increase in impulsivity (such as was found in this experiment). If the 
increase in impulsivity was a result of a decrease in sensitivity to the temporal context how much sensitivity decreases (i.e. how "negative" $k$ becomes) may be a function of how much ethanol sucrose was preferred to plain sucrose. The next experiment investigates this possibility by presenting the subjects from Experiment 3 with concurrent access to ethanol- and plain- sucrose. 


\section{EXPERIMENT 4}

\section{Concurrent Access to Ethanol Sucrose and Plain Sucrose}

The purpose of Experiment 4 was to determine if there was a correspondence between preference for the impulsive $(\mathrm{MN})$ reinforcer alternative in Experiment 3 and preference for ethanol sucrose in a concurrent access situation. When the delay to terminal reinforcer delivery was increased in Experiment 3 the impact the terminal reinforcers had on initial link response distribution decreased. As that impact decreased the behaviour of five of the six subjects shifted towards impulsivity. Since there were qualitatively different reinforcers delivered in the initial link it is possible that the change towards impulsivity reflected a preference for ethanol sucrose (which was designated the impulsive alternative because it was associated with the smaller terminal reinforcer amount). It is possible that the subject that did not change its behaviour (remained self-controlled) in Experiment 3 actually preferred plain sucrose rather than ethanol sucrose. Thus, for that subject, decreasing the impact of the terminal reinforcers on choice did not change initial link behaviour because plain sucrose was associated with the self-controlled alternative. Arguably, a greater tendency to make impulsive choices in the $\mathrm{MN}$ - ML paradigm could reflect a greater preference for ethanol sucrose which can be assessed using concurrentVI VI schedules.

Previous studies have used concurrentVI VI schedules to investigate preference between reinforcer alternatives that differed qualitatively (Foster, Temple, Robertson, Nair, \& Poling, 1996; Matthews \& Temple, 1979; McAdie, Foster, \& Temple, 1996). For those studies, the strength of subjects' preference for one alternative over the 
other was measured by fitting the GML to the results. According to matching theory, when two alternatives are concurrently available subjects will allocate their responses between those alternatives in proportion to the reinforcement rate provided by each schedule (Herrnstein, 1961, 1970). If one of the two schedules becomes richer (i.e. the variable interval decreases so that reinforcer delivery is more frequent), the subject will allocate more of their responses to the richer schedule and decrease their responses on the leaner schedule. Additionally, when the alternatives differ qualitatively that difference will be reflected as bias $(\log \mathrm{c})$ or as a greater tendency to respond on the alternative that delivers the preferred reinforcer regardless of the scheduled contingencies (Hollard \& Davison, 1971). Thus, previous studies have assessed preference for different types of reinforcers by examining bias to reinforcer alternatives. Most of those studies have included one or two conditions where the same reinforcer was delivered on both alternatives. Those conditions were then used to estimate inherent bias for one alternative over the other. That inherent bias is then subtracted from the measures of bias obtained when the reinforcer alternatives delivered different quality reinforcers. For example, McAdie et al. (1996) investigated whether the GML could quantify the aversiveness of a sound stimulus. They presented hens with concurrent VI VI arranged so that an aversive noise the sound of hens in a commercial poultry shed) was associated with one alternative. They found that the GML provided a good description of their data and, by using estimates of inherent bias, they were able to provide a measure of bias for the noisefree alternative without the confound of inherent bias. Two studies with cows found that this species of animal tends towards more extreme undermatching than is typically found in rats (Foster et al., 1996; Matthews \& Temple, 1979) but that preference for one quality of reinforcer over another could be assessed using a GML- 
based analysis. Foster et al. (1996) used a condition in which the same quality food was delivered on both alternatives according to equal VI schedules to estimate the amount of inherent bias the subjects had for one alternative as compared to the other. This then enabled them to determine bias resulting from quality differences by subtracting the estimated inherent bias from the bias found when subjects were given access to two different foods over a series of concurrentVI VI schedules. Similarly, Matthews and Temple (1979) used a GML approach to investigate if individual cows preferred chopped hay or dairy meal. The GML fit their data set well and they found that the cows in their study showed a slight preference for dairy meal, which was ascertained by examining the bias parameter.

The current study examined response allocation between concurrentVI VI schedules to assess whether subjects preferred ethanol sucrose or plain sucrose. One schedule delivered three dips of ethanol sucrose for each interval completed. The other schedule delivered three dips of plain sucrose for each interval completed. The amount of reinforcement delivered for each completed VI was relatively large to ensure that subjects could maintain ethanol intakes $(\mathrm{g} / \mathrm{kg} / \mathrm{hr})$ similar to their intakes in Experiment 3. Three pairs of VI schedules were used concurrent VI 15 s VI 15 s, VI $15 \mathrm{~s}$ VI $5 \mathrm{~s}$, and VI $5 \mathrm{~s}$ VI $15 \mathrm{~s}$. These relatively rich schedules were used because the subjects had previous experience with a high rate of reinforcement in Experiment 3 and there is evidence that suggests that demand for a reinforcer may vary for lean versus rich schedules of reinforcement (Hursh, 1991). Previous research with ethanol as a reinforcer suggests that side bias sometimes varies as reinforcer schedules are varied (Anderson et al., 2002). Thus, a procedural variation was used and each pair of schedules was run twice, firstly with ethanol sucrose associated with the left schedule 
and plain sucrose associated with the right schedule. Secondly, the pair of schedules was run with plain sucrose on the right and ethanol sucrose on the left. This procedural variation was used rather than arranging concurrent VI VI schedules that both delivered the same quality of reinforcer and then using that data to estimate a value for side bias because that standard approach assumes that side bias is a constant between conditions. It was predicted that the rats that became more impulsive as the delay to the delivery of the terminal reinforcers increased in Experiment 3 would be biased towards ethanol sucrose in the current study. It was also predicted that the subject that maintained a constant level of self-control in Experiment 3 would be biased towards plain sucrose.

Method

Subjects

The six subjects that completed Experiment 3 completed Experiment 4. All subjects were kept at $85 \%$ of their free-feeding body weight by post-session feeding. The housing conditions were the same as for Experiment 1.

\section{Apparatus}

The apparatus used in Experiment 3 was used in Experiment 4.

\section{Procedure}

As all six subjects had experience responding on both the left and the right lever no specific pre-training was necessary. Each session began when the front levers were inserted into the chamber and the front lights were turned on. The reinforcer contingency in effect was independent concurrent VI VI schedules. The schedules were arranged by a twelve-interval arithmetic progression and intervals were 
presented randomly without replacement. The variable interval schedules and number of sessions required to meet the stability criteria are listed in Table 5.1. When a programmed interval elapsed, the next response the subject made on the lever associated with that schedule was reinforced with three dips of the appropriate reinforcer. Responses on one of the levers were reinforced with ethanol sucrose from the dipper directly below. Responses on the second lever were reinforced with plain sucrose from the dipper located below that lever. Location of the ethanol sucrose and the plain sucrose varied across conditions (Table 5.1). Reinforcer amount was set at three dips so that the rats could consume volumes of ethanol $(\mathrm{g} / \mathrm{kg} / \mathrm{hr})$ similar to those obtained in Experiment 3. There was a 2-s changeover delay to reduce the likelihood of subjects alternating between reinforcement options (Herrnstein, 1961). Sessions continued until 51 reinforcers had been collected or until 45 minutes had elapsed, whichever came first.

Table 5.1

Order of conditions and location of the ethanol sucrose (ethanol) and the plain sucrose (sucrose) for each condition

\begin{tabular}{cccccc}
\hline \multirow{2}{*}{ Condition } & \multicolumn{2}{c}{ Reinforcer Type } & & & \\
\hline 1 & Left & Right & VI Left & VI Right & Sessions \\
\hline 2 & sucrose & ethanol & 15 & 15 & 13 \\
3 & ethanol & sucrose & 15 & 15 & 16 \\
4 & ethanol & sucrose & 5 & 15 & 17 \\
5 & ethanol & sucrose & 15 & 5 & 10 \\
6 & sucrose & ethanol & 15 & 5 & 14 \\
\hline
\end{tabular}

The stability criteria used was that the moving average of the proportion of responses on the left lever for the two most recent five session blocks had to be with 0.05 of the average from the previous five sessions. A minimum of 10 sessions was required per 
condition and there could be no monotonically increasing or decreasing trend in the proportion of sucrose reinforced responses over the final five sessions.

Results and Discussion

Ethanol Consumption

Data were averaged over the last 5 sessions of each condition. Although the maximum duration of each session was set to 45 minutes, the average time to complete a session was 18.9 minutes (range 15.3 minutes to 23.9 minutes). This meant that the rate of ethanol consumption in this experiment was high compared to previous experiments. Grams per kilogram of ethanol consumed per hour for each condition was calculated using each individual subjects' $85 \%$ target weights (Table 5.2).

Table 5.2

The average amount of ethanol $(\mathrm{g} / \mathrm{kg} / \mathrm{hr})$ consumed by each subject for the last five sessions of each condition

\begin{tabular}{ccccccc}
\hline Condition & E11 & E21 & E31 & E41 & E51 & E61 \\
\hline \hline Ethanol Left Plain Right & & & & & & \\
$\quad$ VI 15 s VI 15 s & 4.32 & 5.55 & 2.86 & 4.46 & 4.20 & 3.98 \\
VI 15 S VI 5 s & 0.14 & 0.04 & 0.04 & 0.04 & 3.83 & 2.71 \\
VI 5 s VI 15 s & 2.60 & 4.41 & 2.60 & 1.86 & 9.00 & 8.48 \\
& & & & & & \\
Plain Left Ethanol Right & & & & & & \\
$\quad$ VI 15 s VI 15 s & 3.42 & 1.57 & 3.38 & 5.13 & 3.84 & 3.73 \\
VI 15 S VI 5 s & 0.65 & 0.70 & 2.64 & 1.40 & 8.39 & 8.43 \\
VI 5 s VI 15 s & 0.09 & 0.22 & 0.16 & 0.19 & 1.39 & 0.10 \\
\hline
\end{tabular}

\section{$\underline{\text { GML Analysis }}$}

The data obtained for each subject in Experiment 4 was plotted with the log ratio of responses as a function of the log ratio of the obtained reinforcers (Figure 5.1). The Generalised Matching Law (Equation 3) was used to analyse the data and the line of best fit was determined using a regression analysis. The results of that fit are 
E11

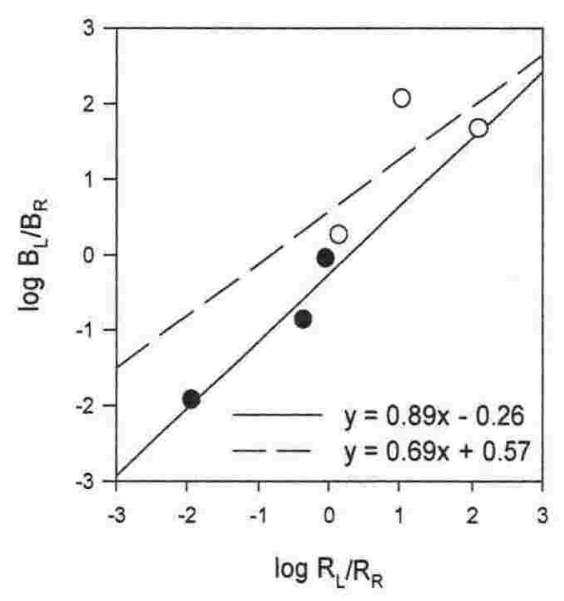

E31

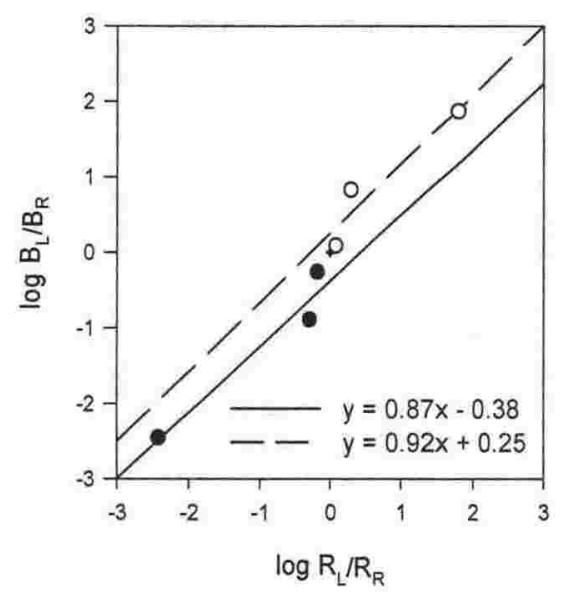

E51

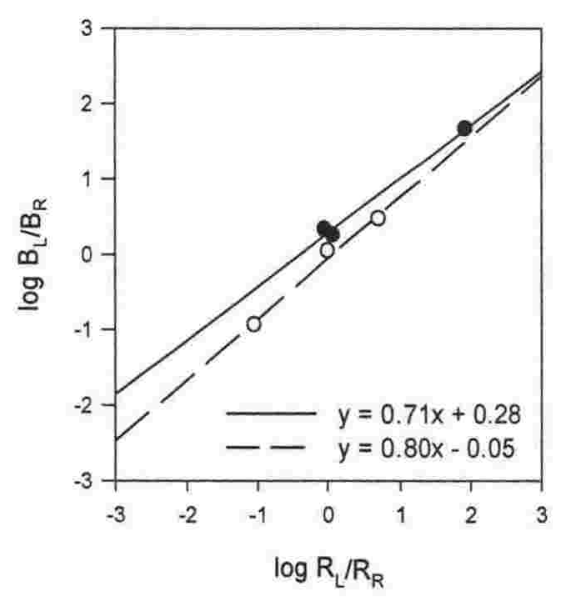

E21

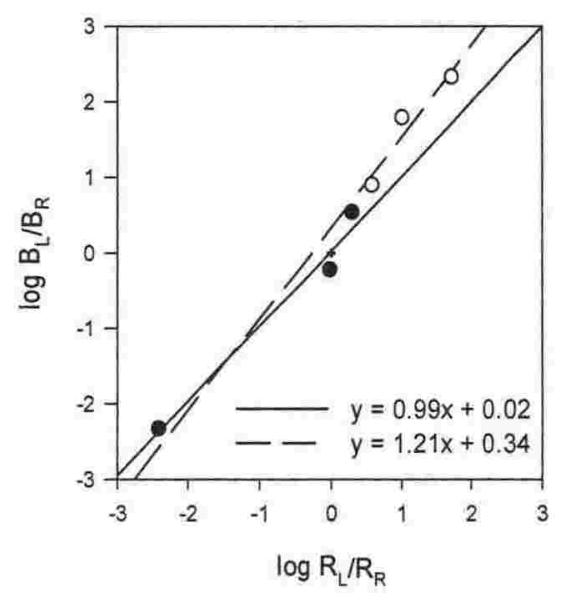

E41

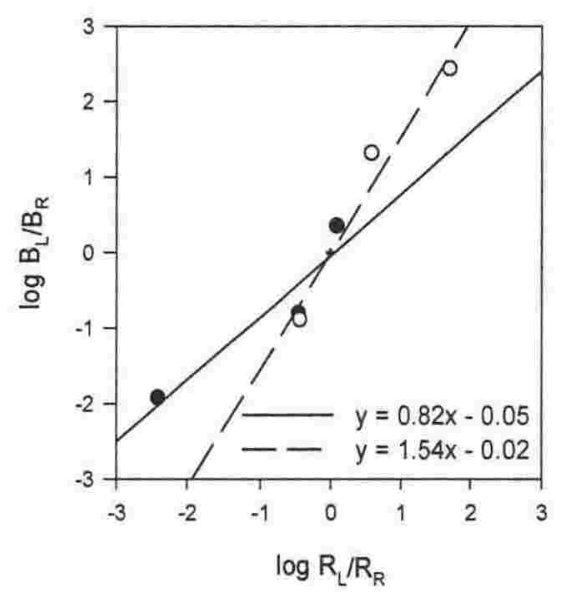

E61

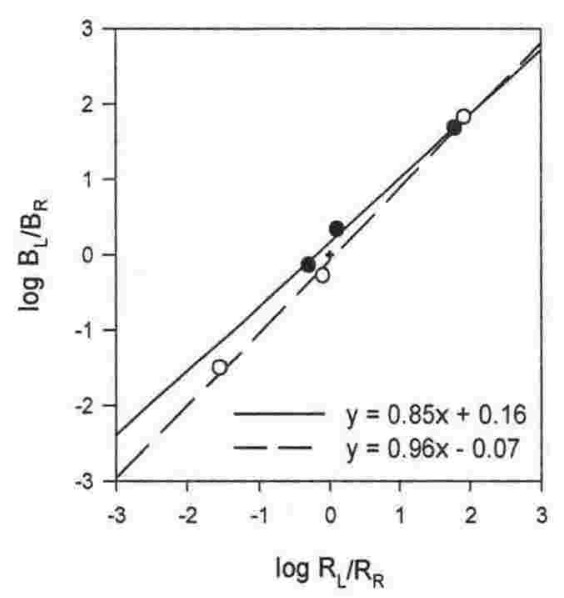

Figure 5.1. Logarithms of the obtained behaviour plotted as a function of the logarithms of the obtained reinforcers. The filled circles represent behaviour when ethanol sucrose was linked to the left and plain sucrose was linked to the right. The open circles are from when the locations of the reinforcers were reversed and ethanol sucrose was associated with the right. The solid lines are the linear regression line for conditions in which ethanol sucrose was on the left; and the dashed lines are the linear regression lines when ethanol sucrose was on the right. The equations for the regression lines are displayed on each graph. A small cross indicates the origin for each graph, this is obscured for subject E11 and E51. 
presented in Table 5.3. The value of the bias parameter varied when the location of the ethanol sucrose and plain sucrose was varied. When ethanol sucrose was on the left a bias for ethanol sucrose resulted in a positive $\log \mathrm{c}$ and bias for plain sucrose resulted in a negative $\log \mathrm{c}$. The reverse was true when ethanol sucrose was on the right and plain sucrose was on the left. In Figure 5.1, the solid line shows the line of best fit for conditions in which ethanol sucrose was on the left and the dashed line shows the line of best fit for conditions in which plain sucrose was on the left. If the dashed line is above the solid line in Figure 5.1 it indicates that the subject preferred the alternative that delivered plain sucrose. Conversely, when the solid line is above the dashed line the subject preferred the alternative that delivered ethanol sucrose. A visual analysis of Figure 5.1 shows that the behaviour of subjects E11, E21, and E31 was biased towards plain sucrose. The behaviour of subject E41 was unbiased. The behaviour of the remaining two subjects (E51 and E61) was biased towards ethanol sucrose.

Table 5.3

Parameters obtained from the GML analysis of the results

\begin{tabular}{ccccccccc}
\hline \multicolumn{3}{c}{ Ethanol sucrose on Left } & \multicolumn{4}{c}{ Ethanol sucrose on Right } \\
Subject & $\mathrm{a}$ & $\log \mathrm{c}$ & $\mathrm{r}^{2}$ & Subject & $\mathrm{a}$ & $\log \mathrm{c}$ & $\mathrm{r}^{2}$ \\
\hline \hline E11 & 0.89 & -0.26 & 0.92 & E11 & 0.69 & 0.57 & 0.51 \\
E21 & 0.99 & 0.02 & 0.97 & E21 & 1.21 & 0.34 & 0.92 \\
E31 & 0.87 & -0.38 & 0.94 & E31 & 0.92 & 0.25 & 0.91 \\
E41 & 0.81 & -0.05 & 0.90 & E41 & 1.54 & -0.02 & 0.95 \\
E51 & 0.71 & 0.28 & 0.99 & E51 & 0.81 & -0.05 & 0.99 \\
E61 & 0.85 & 0.16 & 100.00 & E61 & 0.96 & -0.07 & 100.00 \\
\hline
\end{tabular}

Although two of the three subjects (E51 and E61) that were the most impulsive in Experiment 3 were biased towards ethanol sucrose in the current study, the third subject (E21) that was relatively more impulsive than the others was biased towards plain sucrose. Two other subjects (E11 and E31) made very few impulsive responses 
in Experiment 3 and were biased towards plain sucrose in the current study. The remaining subject (E41) was indifferent to reinforcer quality despite showing an increase in impulsivity as delay increased in Experiment 3. A quantitative measure of quality bias can be obtained by first determining the midpoint on the y-axis between the intercepts of the lines of best fit for conditions when ethanol sucrose was on the left and when it was on the right. The distance that each line is from the midpoint of the two functions is a measure of how biased that subject was towards one alternative over the other. If the intercept of the dashed line is above the intercept of the solid line the quality bias is for plain sucrose; and, if the intercept of the solid line is above the intercept for the dashed line the quality bias is for ethanol sucrose. The quality bias measures were assigned negative values when the bias was for plain sucrose and positive values when the bias was for ethanol sucrose. Table 5.4 shows quality bias measures for each subject along with the average proportion of $\mathrm{MN}$ choices each subject made in Experiment 3 and the derived values of the sensitivity to temporal context measure $(k)$ derived using the CCM in Experiment 3.

Table 5.4

Individual quality bias values from Experiment 4; and average proportion of impulsive choices and sensitivity to temporal context $(k)$ values from Experiment 3

\begin{tabular}{cccc}
\hline Subject & Quality Bias & Avg Prop MN & $k$ \\
\hline E11 & -0.42 & 0.02 & 0.19 \\
E21 & -0.16 & 0.11 & -0.48 \\
E31 & -0.32 & 0.04 & -0.89 \\
E41 & -0.02 & 0.02 & -1.26 \\
E51 & 0.17 & 0.11 & -1.22 \\
E61 & 0.12 & 0.16 & -2.21 \\
\hline
\end{tabular}

There was a positive correlation $(r=.69)$ between the value of the quality bias and the proportion of MN responses made by subjects in Experiment 3. As the value of the quality bias increases, bias towards ethanol sucrose increases, thus, the subjects that 
were more biased towards ethanol sucrose in the current experiment were generally more impulsive in Experiment 3. A negative correlation $(\mathrm{r}=-.81)$ was found between the quality bias measure in the current experiment and sensitivity to the temporal context ratio (the scaling parameter, $k$ ) in Experiment 3.

Smaller (negative) $k$ values in Experiment 3 meant that as the temporal context parameter increased in value, its impact on behaviour was moderated by the sensitivity parameter so that the effect of temporal context on response allocation in the initial link decreased. Thus, the current results indicate that the less a subject's behaviour was influenced by temporal context in Experiment 3 the less biased they were towards plain sucrose in Experiment 4. This suggests that the impact of the different quality initial link reinforcers in Experiment 3 was to change the value of $k$. That is, reinforcer quality in the initial link reduced sensitivity to the temporal context.

The less sensitive initial link behaviour is to temporal context, the less impact the terminal link contingencies will have on that behaviour. When there are no primary reinforcers delivered in the initial link of concurrent chains schedules initial link behaviour distribution is determined by a combination of the terminal link reinforcement contingencies and the conditioned reinforcers with which they are associated. The effect of temporal context in such situations is that it increases the impact that terminal link contingencies have on initial link behaviour as the absolute duration of the terminal link increases. Terminal reinforcer contingencies have a greater impact on initial link behaviour as terminal link duration increases because subjects spend more time in the presence of the conditioned reinforcers that are associated with each terminal link. 
The effect of adding initial link reinforcers in Experiment 3 was to reduce the impact that the terminal reinforcer contingencies had on initial link responding. The longer the terminal link durations became the less impact the terminal link reinforcers had on initial link choice. This decrease in impact can be modelled using the $\mathrm{CCM}$ when the temporal context sensitivity parameter $(\mathrm{k})$ is allowed to vary. Negative values of $\mathrm{k}$ describe situations in which increasing the value of the temporal context parameter decreases initial link sensitivity to terminal link contingencies. Therefore, the effect of delivering qualitatively unequal reinforcers in the initial link of the MN - ML paradigm used in Experiment 3 can be characterised in terms of a change in sensitivity to the temporal context. This is consistent with Grace's (1994) suggestion that temporal context is moderated by procedural variables. Thus, the use of the additional free parameter $k$ is justifiable as a measure of the effect reinforcer quality has on initial link response distribution in the MN - ML analogue of self-control behaviour. 


\section{GENERAL DISCUSSION}

The two central issues for this thesis were self-control and reinforcer quality. The two reinforcer qualities used were sweetened solutions that were either ethanol free or mixed to a concentration of $10 \%$ ethanol. Ethanol was chosen to manipulate reinforcer quality because there is a body of literature that suggests that subjects responding for ethanol are responding to gain access to the pharmacological effects of the drug (Files et al., 1995; Heyman, 1993). Using ethanol to manipulate the quality of the reinforcer alternatives enables comparisons between the current results and the results of other studies that have investigated drugs as reinforcers. The pharmacological effects of ethanol were assumed to change the quality of the sweetened solutions by altering their reinforcing value.

The first experiment examined the effect of different reinforcer qualities on choice in a self-control paradigm. The paradigm was based on traditional self-control analogues where subjects chose between two reinforcer alternatives. In those paradigms, the choice is to receive either a small amount of reinforcer after a brief delay or a large amount of reinforcer after a longer delay. Choosing the small reinforcer is impulsive because doing so reduces the amount of reinforcement a subject can earn on each trial. Opting for the large reinforcer is self-control because, although the delay to reinforcer delivery is longer, choosing that option increases the amount of reinforcement earned on each trial. In the first phase of the study reported here, the quality of the SI and the LD were equal and half the subjects were reinforced with plain sucrose and the remaining subjects were reinforced with ethanol sucrose. Three delay ratios were studied. When the reinforcer qualities were equal (Phase I), 
subjects' behaviour allocation in the initial link tended to be more impulsive at the most extreme delay ratio (1:6) than at the least extreme delay ratio (1:2). However, the predicted monotonic increase in impulsivity was not found for any of the subjects. The delay ratio conditions were then repeated (Phase II) with ethanol sucrose delivered following each impulsive response and plain sucrose delivered for each selfcontrolled response. The data set obtained in that phase of Experiment 1 was more orderly than in Phase I. Four of the six subjects monotonically increased the proportion of impulsive choices they made as the delay ratio became more extreme. A possible explanation for the more systematic behaviour changes found in Phase II is that having a different quality of reinforcer delivered for SI versus LD choices made the reinforcer contingencies more discriminable by increasing sensitivity to the differences between the alternatives. Sensitivities to the two independent variables (the amount ratio, $\mathrm{a}_{\mathrm{A}}$, and the delay ratio, $\mathrm{a}_{\mathrm{D}}$ ) were determined using the GCML and the CCM. Although both models described the data set well, neither of these analyses revealed a systematic effect of reinforcer quality on the individual sensitivity parameters.

Another way to consider the data from Phase I and Phase II was to determine if there were systematic changes in the sensitivity ratio $\left(a_{A} / a_{D}\right)$ when the reinforcer quality was changed (Chelonis \& Logue, 1997). Decreases in the sensitivity ratio should be associated with increases in the proportion of impulsive choices in Phase II versus Phase I; and, increases in the sensitivity ratio should be associated with decreases in the proportion of impulsive choices. The behaviour of the majority of the subjects adhered to this pattern and negative correlations were found between the sensitivity ratio and impulsivity for both phases of the experiment. This result indicates that 
higher sensitivity ratios were associated with lower impulsivity, which supports the use of the sensitivity ratio as a measure of impulsivity in traditional SI - LD selfcontrol paradigms. This finding held true when either the GCML or the CCM were used to analyse the data. The results indicate that one way reinforcer quality affected behaviour in this experiment was that the concurrently available reinforcer alternatives were more discriminable. This is consistent with Davison and Jenkins (1985) suggestion that increasing the disparity between the stimuli associated with reinforcer alternatives should make behaviour more sensitive to the reinforcement contingencies. Finally, although SI - LD paradigms have been successfully used to examine a variety of choice alternatives, they do not address the choice situation of most relevance for this thesis.

One aim of this thesis was to develop an animal paradigm to study self-control that addressed situations that are not covered by existing paradigms. This thesis addressed situations in which an individual must choose between two immediately available reinforcers of unequal quality and value that have mutually exclusive contingent delayed reinforcers of equal quality but unequal value. For example, if a person is trying to maintain their weight by dieting, that person will repeatedly encounter situations in which two (or more) food reinforcers are available. Each time they must choose between a low calorie (e.g. an apple) and a high calorie (e.g. cheesecake) option their decision has both immediate and delayed consequences. If the person really preferred cheesecake but chose the apple they would be acting with self-control. The immediate consequence of that decision would be forfeiting the most valuable immediate reinforcer (the cheesecake). However, the delayed consequences of the choice would be weight loss; and the delayed consequences of choosing the 
cheesecake would be weight gain. It is this type of situation in which individuals chose between having more now (the most valued immediate reinforcer) or more later (the most valued delayed reinforcer) that the model of choice behaviour developed for this thesis was designed to address.

The MN - ML self-control paradigm developed for this thesis delivers primary reinforcement after two delays for each trial. The first primary reinforcer was delivered immediately after the initial link schedule requirement had been met. The second was delivered after a brief delay. In Experiment 2, the immediately delivered reinforcer was a single dip of either plain milk or ethanol milk. Choosing the plain milk was designated the self-control choice and was followed by delivery of three further dips of reinforcer. The impulsive choice was responding to receive ethanol milk immediately and, after the delay, only one more dip of reinforcer was delivered for that trial. The quality of the delayed reinforcers was plain sucrose in one phase of the experiment and ethanol sucrose in the other phase.

The main finding from Experiment 2 was that response allocation in the initial link varied as a function of both delay to terminal reinforcer delivery and terminal reinforcer quality. In general, increasing the delay to terminal reinforcer delivery increased the number of impulsive choices subjects made. When the terminal reinforcer quality was plain sucrose, twelve of the thirteen subjects became more impulsive as the delay to the terminal links increased. Changing the quality of the terminal reinforcers to ethanol milk had a systematic effect on the behaviour of all of the subjects. All subjects allocated more of their responses to the self-controlled alternative when the terminal reinforcers were ethanol milk. However, the impact of 
increasing the delay to terminal reinforcer delivery was inconsistent between subjects. Eight of the thirteen subjects increased their responding on the impulsive option as delay increased; and, the remaining five subjects decreased their responding to that alternative.

A possible explanation for the differential results is that not all subjects preferred ethanol sucrose over plain sucrose. Subjects that preferred ethanol sucrose were expected to become more impulsive as delay to terminal reinforcer delivery increased because, according to theories of temporal discounting, increasing the delay to the terminal reinforcers should decrease their value (e.g. Rachlin \& Green, 1972). This means that the initial link reinforcers gain relatively more control over initial link response distribution as the delay to the terminal reinforcers increases. However, if subjects preferred plain sucrose to ethanol sucrose, increasing delay to the terminal reinforcers could decrease impulsivity. Again, this would be because increasing the delay to the terminal reinforcers decreased their value and behaviour was more sensitive to the initial link reinforcer contingencies.

When the data from this experiment is compared with previous findings obtained using SI - LD paradigms the pattern of behaviour change is different. A common finding from SI - LD paradigms is that, as in Experiment 2, if the delay ratio is constant at 1:1 and the absolute delay to the terminal reinforcer delivery is increased subjects become more self-controlled (e.g., Ito \& Asaki, 1982). Thus, the introduction of primary reinforcers in the initial link of concurrent-chains schedules appears to change response distribution. In a previous study Davison and Smith (1986) found that when the concurrent schedules were nonindependent, and primary 
reinforcer delivery occurred immediately following schedule completion and after a delay, subjects were insensitive to the terminal link reinforcer contingencies. In the Davison and Smith study all reinforcers were of the same quality and terminal link reinforcer delivery was controlled by a VI 30 -s schedule. It is possible that subjects in Davison and Smith's study were insensitive to the terminal link reinforcer rates when there was reinforcement in the initial links because primary reinforcement would not have been delivered in the terminal link of each trial. Support for this is found in the fact that when subjects were presented with a choice between only the delayed SI and LD reinforcers they ceased responding and the initial link VI schedules needed to be changed because subjects were extremely biased towards the SI alternative.

Experiment 2 of this thesis was dissimilar to Davison and Smith's study because the reinforcers delivered upon completion of the schedule requirements were of unequal qualities. In Experiment 1, the introduction of unequal quality reinforcers appeared to change the discriminability of the terminal link reinforcement, which resulted in behaviour that was more sensitive to the reinforcer contingencies.

A problem that was found in Experiment 2 was that subjects responding in the $\mathrm{MN}-$ ML paradigm tended to develop significant side biases. Experiment 3 was designed to try to minimise side bias. The modified $\mathrm{MN}-\mathrm{ML}$ procedure was then used to collect data from more delay conditions to make it possible to fit quantitative models to the data. The CGML and CCM predict different results when the delay ratio is held constant at 1:1 and the absolute durations of the delays increases. According to the GCML, it is the relative rather than the absolute delays that control initial link response distribution. Thus, the GCML predicts that behaviour will not change when the delay to delivery of the terminal reinforcers increased. In contrast, the CCM 
assumes that behaviour is a function of both the delay to reinforcement delivery and temporal context. Increasing the delay to terminal link delivery changes the temporal context. This is because increasing the delay also increases the duration of the terminal links relative to the duration of the initial links. Thus, CCM predicts that behaviour distribution would change when the delay was changed. What the CCM was unable to predict was the direction of the change in initial link behaviour when primary reinforcement was delivered in both the initial and the terminal links of a concurrent-chains procedure. The results of Experiment 3 were that the behaviour of five of the six subjects became more impulsive as the absolute delay to terminal reinforcer delivery increased. Thus, the GCML was unable to account for the results of that experiment. The CCM was able to account for the results when the temporal context scaling parameter $(k)$ was allowed to vary. The shift in the direction of impulsivity with increasing delay was described by negative $k$ values. The $k$ values were negatively correlated with the average proportion of impulsive choices subjects made across the three delay conditions. This indicates that the more impulsive a subject was the less sensitive its behaviour was to temporal context. As sensitivity to temporal context decreases the impact that the terminal link reinforcer contingencies exert on initial link behaviour decreases. The behaviour shift towards impulsivity in Experiment 3 was in the opposite direction to behaviour changes seen in concurrent chains schedules when primary reinforcement is only delivered in the terminal links (White \& Pipe, 1987).

For Experiment 2 and Experiment 3, initial link behaviour was a function of both the initial link reinforcers and the terminal link reinforcers. The effect of adding primary reinforcers to the end of the initial link was that subjects were not as sensitive to the 
terminal-link reinforcement contingencies as they would have been otherwise. It is not clear if the decrease in sensitivity to the temporal context resulted from the addition of primary reinforcement to the initial links, or from the use of unequal reinforcer qualities in the initial link, or from a combination of these factors. Future research could address this question by systematically varying the reinforcer contingencies associated with the initial and terminal links. For instance, behaviour could be studied when all of the reinforcers delivered in the $\mathrm{MN}-\mathrm{ML}$ paradigm were of equal quality and compared to behaviour when the initial links deliver different quality reinforcers. If the presence of initial link reinforcers alone changes sensitivity to the temporal context then behaviour would be predicted to shift from self-control towards indifference as the delay to delivery of the terminal reinforcers increases when equal quality reinforcers were used. Such a shift could be described by a negative $k$ value. If, however, differential reinforcer quality is necessary to change sensitivity to temporal context, behaviour in the MN - ML paradigm should favour the self-controlled (ML) alternative at brief delays. The extent to which the ML alternative was preferred to the $\mathrm{MN}$ alternative would then be expected to increase as the delay to delivery of the terminal reinforcers increased because, as long as sensitivity to the temporal context remains a positive value, the CCM predicts a shift towards greater self-control as the duration of the terminal link increases.

In summary, the MN - ML procedure used in Experiment 2 and Experiment 3 has some unique features that are not included in more traditional SI - LD paradigms. The inclusion of primary reinforcement in the initial links addresses choice situations in which reinforcement for behaviour is a "package" of immediate and delayed consequences. A noteworthy finding was that behaviour in the initial link became 
more impulsive as the delay to the terminal reinforcers increased. This finding is notable because previous studies that did not include initial link reinforcers found that impulsivity decreased when delay increased. Moreover, this result has implications for the treatment of impulsive behaviour disorders, such as alcohol dependence, because it highlights the need to alter the subjective value of more highly valued immediate reinforcers (e.g. alcohol) that impact on the attainment of more highly valued delayed reinforcers (e.g. sobriety). Another important finding was that, unlike the GCML, the CCM was able to account for the results of Experiment 3 when the scaling parameter was included in the model. This finding is important because it indicates that the impact of temporal context on response distribution is influenced by reinforcer quality. The CCM analysis also indicates that reinforcer quality can be incorporated into quantitative models differently than other independent variables such as reinforcer amount or immediacy. Furthermore, the analysis demonstrated that the CCM, which was developed to account for behaviour from concurrent-chains schedules that have concurrent VI VI initial links, was able to describe data obtained from a procedure that had concurrent FI FI initial links.

There are some limitations to the $\mathrm{MN}-\mathrm{ML}$ paradigm. The first issue was that significant side biases developed without the trial blocking procedure. Future research using this paradigm will have to include that procedure. Another procedural drawback was the use of non-independent concurrent schedules, which meant that terminal-link entry rate was not equal across alternatives. A further limitation of the $\mathrm{MN}$ - ML paradigm is that many of the situations it was designed to emulate require repeated choices of less valued immediate reinforcers before the more valued reinforcer is delivered. For instance, in the healthy eating example, the consequence 
of choosing a single apple for someone who habitually consumes cheesecake will not be weight loss; it is necessary for that person to frequently choose fruit over sweets to achieve their goal. A modification of the procedure, which could address this situation, would be to set up reinforcement contingencies so that the delivery of a preferred reinforcer did not occur until a subject had repeatedly responded to receive less valuable immediate reinforcers. There is some evidence that suggests if behaviour is reinforced with "bundles" of reinforcer the delayed contingencies become more salient and self-controlled responding increases (Ainslie \& Monterosso, 2003). It might therefore be possible to investigate the impact of varying the pattern of response requirement on choice behaviour. Of particular interest are situations in which responding access to a preferred delayed reinforcer is dependent on repeated choices of less valuable immediate reinforcers. Additionally, contingencies could be arranged in which each response for the most valuable immediate reinforcer would increase the delay to the preferred delayed reinforcer. Such conditions are increasingly accurate analogues of human choice behaviour.

One behavioural situation that the $\mathrm{MN}$ - ML paradigm can be used to study is addiction or drug dependence. In such situations, the individual makes choices to consume either drug-free or drug-containing reinforcers. A limitation of the reinforcer qualities studied Experiment 2 and 3 is that it was assumed that subjects would prefer ethanol sucrose to plain sucrose. The increase in impulsive responding seen in those two experiments could have resulted from either a preference for ethanol-sucrose over plain sucrose, or from indifference to the qualitative differences. The fact that only two of the six subjects that completed Experiment 3 were biased towards ethanol-sucrose in Experiment 4 indicates that it is possible that the 
behaviour change seen in the MN -ML paradigms was due to addition of initial link reinforcers rather than due to differential reinforcer quality per se. A future research possibility would be to systematically examine how varying reinforcer quality influences sensitivity to the temporal context. One approach would be to select several different qualities of reinforcers and present subjects with reinforcer pairs to develop individually scaled ratings of reinforcer quality (Miller, 1976). The individual ratings could be used to select the most preferred and least preferred reinforcer qualities, which could be then be used in the $\mathrm{MN}$ - ML paradigm.

In conclusion, the results obtained using the novel $\mathrm{MN}$ - ML paradigm supports its use as a method to study self-control. The findings suggest that the addition of initial link reinforcers to a concurrent-chains procedure influenced sensitivity to the temporal context that, in turn, influences sensitivity to reinforcer amount. When the initial link reinforcers were of equal amounts but differed qualitatively, the impact of the terminal reinforcers on choice decreased as delay to delivery of those reinforcers increased. The data obtained from the $\mathrm{MN}-\mathrm{ML}$ procedure could not be accounted for by the GCML because behaviour distribution varied as a function of the absolute delay to terminal reinforcer delivery. The CCM described the data well and was able to account for the increase in impulsivity that occurred as delay increased. The results from the $\mathrm{MN}$ - ML paradigm indicated that the impact of differential reinforcer quality in the initial links was to decrease sensitivity to the temporal context. The reduction in sensitivity to temporal context resulted in behaviour that became more impulsive as the delay to terminal reinforcer delivery increased. Additionally, findings from the first experiment indicate that terminal reinforcer contingencies are more easily discriminated when the reinforcers are of unequal quality. 


\section{APPENDIX A}

These figures are data from all six delay conditions in Experiment 1 Phase I when the SI and LD reinforcer qualities were equal. The white bars are data from the 1:2 delay ratio conditions, the grey bars for the 1:4 conditions, and the black bars for the 1:6 conditions. Subjects F12, F32, and F52 were reinforced with ethanol sucrose; and, subjects F22, F42, and F62 were reinforced with plain sucrose. The figures plot the proportion of responses made on the left alternative for each delay condition. Subject F42 data shows bias free responding while subject F52 is biased, most obviously at the $1: 4$ and $4: 1$ delay conditions.

F12

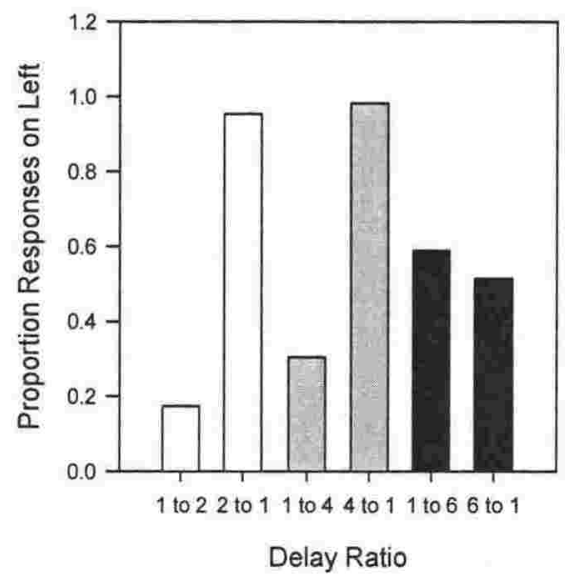

F52

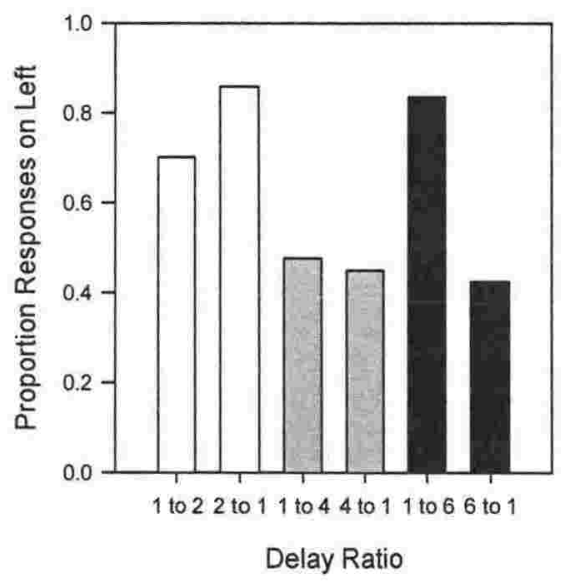

F42

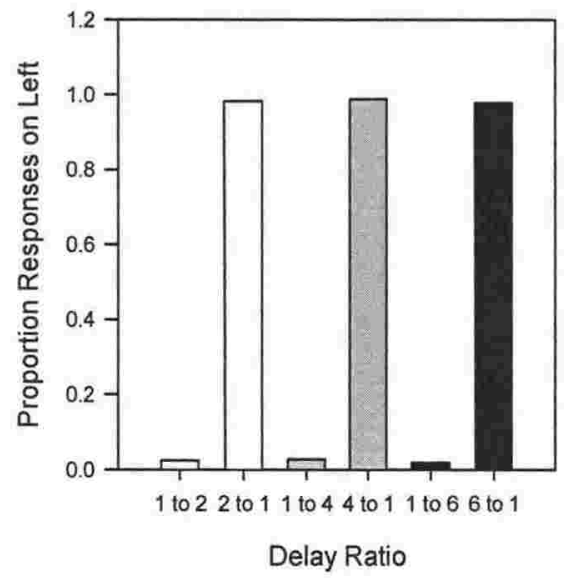

F32

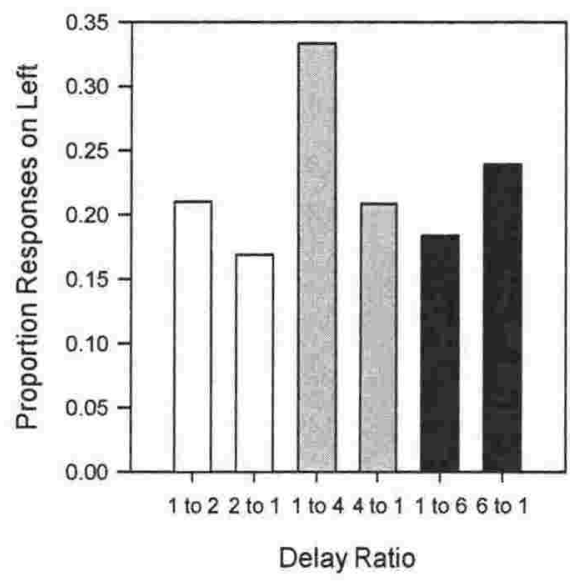

F22

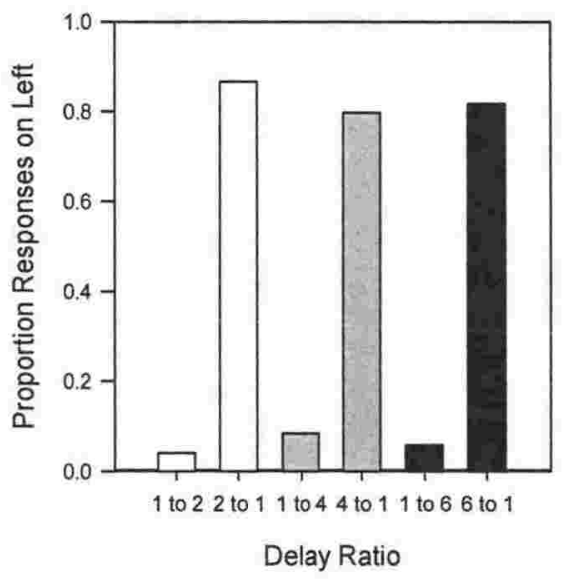

F62

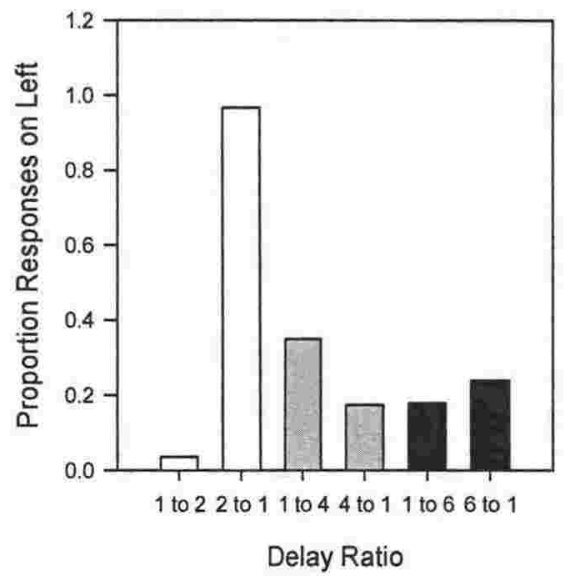




\section{APPENDIX B}

These figures are data from all six delay conditions in Experiment 1 Phase II when the SI reinforcer quality was ethanol sucrose and LD reinforcer quality was plain sucrose. were equal. The white bars are data from the 1:2 delay ratio conditions, the grey bars for the 1:4 conditions, and the black bars for the 1:6 conditions. The figures plot the proportion of responses made on the left alternative for each delay condition.

F12

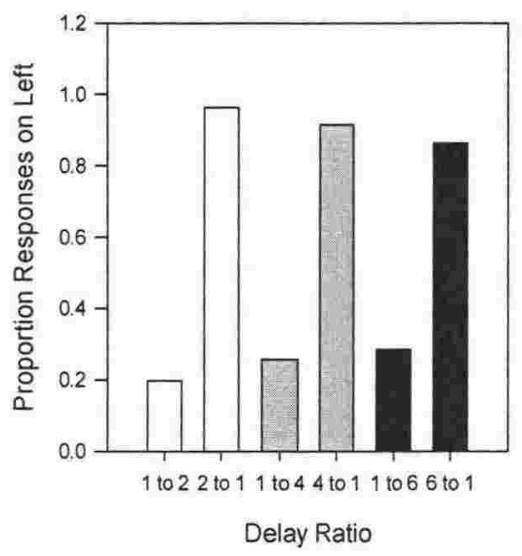

F52

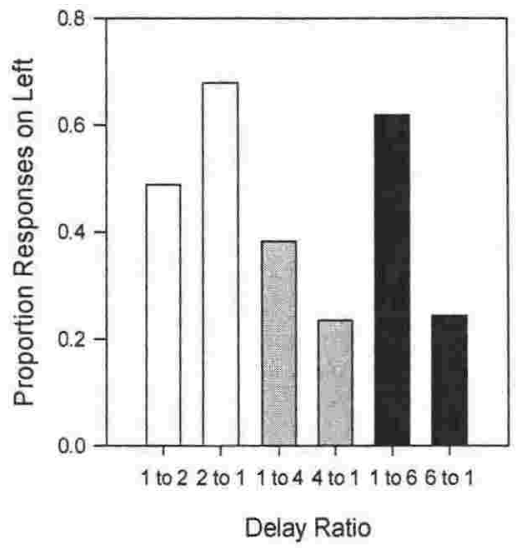

F42

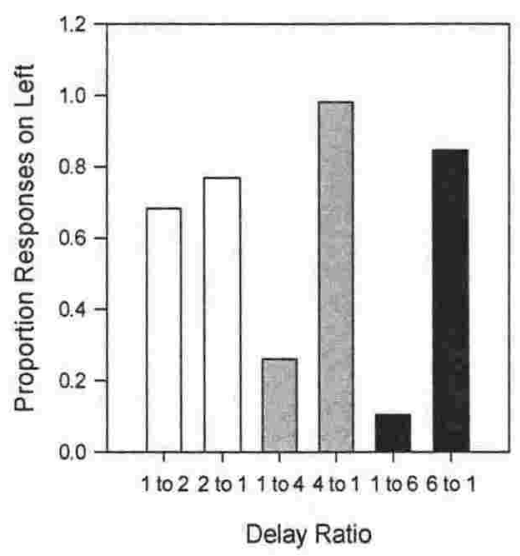

F32

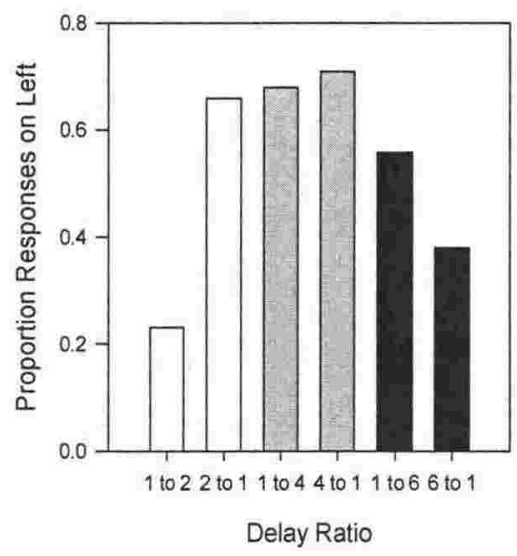

F22

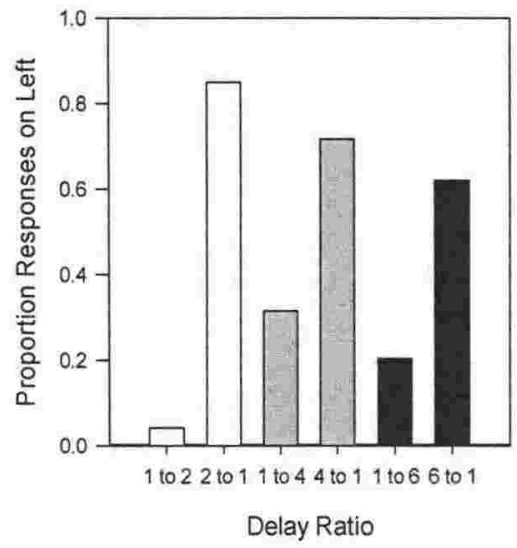

F62

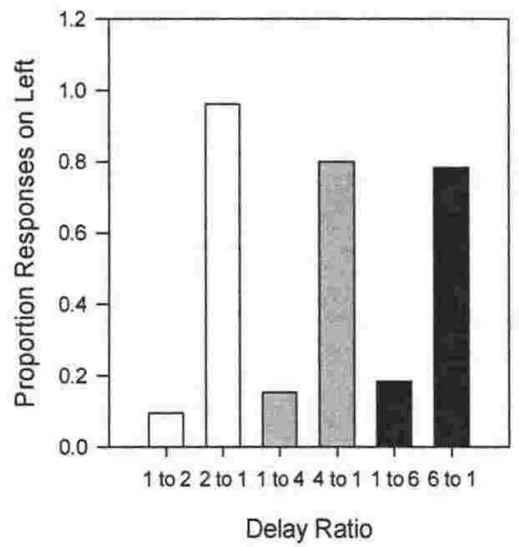




\section{REFERENCES}

Ainslie, G. (1974). Impulse control in pigeons. Journal of the Experimental Analysis of Behavior, 21(3), 485-489.

Ainslie, G., \& Monterosso, J. R. (2003). Building blocks of self-control: increased tolerance for delay with bundled rewards. Journal of the Experimental Analysis of Behavior, 79(1), 37-48.

American Psychiatric Association. (1994). Diagnostic and statistical manual of mental disorders $\left(4^{\text {th }}\right.$ ed.). Washington, DC: Author.

Anderson, K. G., Velkey, A. J., \& Woolverton, W. L. (2002). The generalized matching law as a predictor of choice between cocaine and food in rhesus monkeys. Psychopharmacology, 163, 319-326.

Anderson, K. G., \& Woolverton, W. L. (2003). Effects of dose and infusion delay on concaine self-administration choice in rhesus monkeys. Psychopharmacology, $167,424-430$.

Baum, W. M. (1974). On two types of deviation fom the matching law: bias and undermatching. Journal of the Experimental Analysis of Behavior, 22(1), 231242.

Baum, W. M. (1979). Matching, undermatching, and overmatching in studies of choice. Journal of the Experimental Analysis of Behavior, 32(2), 269-281.

Baum, W. M., \& Rachlin, H. (1969). Choice as time allocation. Journal of the Experimental Analysis of Behavior, 12(6), 861-874.

Beardsley, P. M., Lemaire, G. A., \& Meisch, R. A. (1993). Persistence of ethanol selfadministration as a function of inter-reinforcer interval and concentration. Drug and Alcohol Dependence, 34, 71-81.

Catania, A. C. (1963). Concurrent performances: Reinforcement interaction and response independence. Journal of the Experimental Analysis of Behavior, 6(2), 253-263.

Chelonis, J. J., \& Logue, A. W. (1997). Effects of reinforcer type on rats' sensitivity to variation in reinforcer amount and reinforcer delay. Behavioural Processes, $39,187-203$.

Czachowski, C. L., \& Samson, H. H. (1999). Breakpoint determination and ethanol self-administration using an across-session progressive ratio procedure in the rat. Alcoholism: Clinical and Experimental Research, 23, 1580-1586.

Czachowski, C. L., Samson, H. H., \& Denning, C. E. (1999). Independent ethanoland sucrose-maintained responding on a multiple schedule of reinforcement. Alcoholism: Clinical and Experimental Research, 23(3), 398-403. 
Davison, M. (1983). Bias and sensitivity to reinforcement in a concurrent-chain schedule. Journal of the Experimental Analysis of Behavior, 40(1), 15-34.

Davison, M., \& Jenkins, P. E. (1985). Stimulus discriminability, contingency discriminability, and schedule performance. Animal Learning and Behavior, 13(1), 77-84.

Davison, M., \& McCarthy, D. (1988). The Matching Law: A Research Review. Hillsdale New Jersey: Lawrence Erlbaum Associates.

Davison, M., \& Smith, C. (1986). Some aspects of preference between immediate and delayed periods of reinforcement. Journal of the Experimental Analysis of Behavior, 12(3), 291-300.

Davison, M., \& Tustin, R. D. (1978). The relation between the Generalized Matching Law and Signal-detection Theory. Journal of the Experimental Analysis of Behavior, 29(2), 331-336.

Dziadosz, T., \& Tustin, R. D. (1982). Self-control: an application of the Generalized Matching Law. American Journal of Mental Deficiency, 86(6), 614-620.

Ettinger, R. H., McSweeney, F. K., \& Norman, W. D. (1981). Contrast and undermatching as a function of reinforcer duration and quality during multiple schedules. Journal of the Experimental Analysis of Behavior, 35, 271-282.

Fantino, E. (1969). Choice and rate of reinforcement. Journal of the Experimental Analysis of Behavior, 12(5), 723-730.

Fantino, E., \& Herrnstein, R. J. (1968). Secondary reinforcement and number of primary reinforcements. Journal of the Experimental Analysis of Behavior, 11(1), 9-14.

Farrar, A. M., Kieres, A. K., Hausknecht, K. A., de Wit, H., \& Richards, J. B. (2003). Effects of reinforcer magnitude on an animal model of impulsive behavior. Behavioural Processes, 64, 261-271.

Files, F. J., Samson, H. H., \& Brice, G. T. (1995). Sucrose, ethanol, and sucrose/ethanol reinforced responding under variable interval schedules of reinforcement. Alcoholism: Clinical and Experimental Research, 19, 12711278.

Foster, T. M., Temple, W., Robertson, B., Nair, V., \& Poling, A. (1996). Concurrentschedule performance in dairy cows: persistent undermatching. Journal of the Experimental Analysis of Behavior, 65(1), 57-80.

Grace, R. C. (1994). A contextual model of concurrent-chains choice. Journal of the Experimental Analysis of Behavior, 61, 113-129.

Grace, R. C. (1995). Independence of reinforcement delay and magnitude in concurrent chains. Journal of the Experimental Analysis of Behavior, 63(3), 255-276. 
Grace, R. C. (1999). The matching law and amount-dependent exponential discountig as accounts of self-control choice. Journal of the Experimental Analysis of Behavior, 71(1), 27-44.

Grace, R. C., \& Nevin, J. A. (1999). Timing and choice in concurrent chains. Behavioural Processes, 45, 115-127.

Green, L., \& Estle, S. J. (2003). Preference reversals with food and water reinforcers in rats. Journal of the Experimental Analysis of Behavior, 79, 233-242.

Green, L., \& Fisher, E. B. J. (2000). Economic substitutability: Some implications for health behavior. In W. K. Bickel \& R. E. Vuchinich (Eds.), Reframing Health Behavior Change with Behavioural Economics (pp. 115-144). Mahwah, New Jersey: Lawrence Erlbaum Associates, Publishers.

Green, L., Fisher, E. B. J., Perlow, S., \& Sherman, L. (1981). Preference reversal and self-control: choice as a function of reward amount and delay. Behaviour Analysis Letters, 1, 43-51.

Green, L., Myerson, J., Holt, D. D., Slevin, J. R., \& Estle, S. J. (2004). Discounting of delayed food rewards in pigeons and rats: is there a magnitude effect? Journal of the Experimental Analysis of Behavior, 81, 39-50.

Green, L., Myerson, J., \& Ostaszewski, P. (1999). Discounting of delayed rewards across the life span: age difference in individual discounting functions. Behavioural Processes, 46, 89-96.

Green, L., \& Snyderman, M. (1980). Choice between rewards differing in amount and delay: toward a choice model of self-control. Journal of the Experimental Analysis of Behavior, 34(2), 135-147.

Grosch, J., \& Neuringer, A. (1981). Self-control in pigeons under the Mischel Paradigm. Journal of the Experimental Analysis of Behavior, 35, 3-21.

Herrnstein, R. J. (1970). On the Law of Effect. Journal of the Experimental Analysis of Behavior, 13(2), 243-266.

Herrnstein, R. J. (1974). Formal properties of the matching law. Journal of the Experimental Analysis of Behavior, 21, 159-164.

Heyman, G. M. (1993). Ethanol regulated preference in rats. Psychopharmacology, $112,259-269$.

Heyman, G. M., \& Oldfather, C. M. (1992). Inelastic preference for ethanol in rats: an analysis of ethanol's reinforcing effects. Psychological Science, 3, 122-130.

Higa, J. J., \& McSweeney, F. K. (1987). Behavioral contrast in rats when qualitatively different reinforcers are used. Behavioural Processes, 15, 131-142.

Hollard, V., \& Davison, M. C. (1971). Preference for qualitatively different reinforcers. Journal of the Experimental Analysis of Behavior, 16, 375-380. 
Hursh, S. R. (1980). Economic concepts for the analysis of behavior. Journal of the Experimental Analysis of Behavior, 34(2), 219-238.

Hursh, S. R. (1991). Behavioral econimics of drug self-administration and drug abuse policy. Journal of the Experimental Analysis of Behavior, 56(2), 377-393.

Hursh, S. R., \& Fantino, E. (1973). Relative delay of reinforcement and choice. Journal of the Experimental Analysis of Behavior, 19(3), 437-450.

Ito, M., \& Asaki, K. (1982). Choice behavior of rats in a concurrent-chains schedule: amount and delay of reinforcement. Journal of the Experimental Analysis of Behavior, 37(3), 383-392.

Johnson, M. W., \& Bickel, W. K. (2002). Within subject comparison of real and hypothetical monetary rewards in delay discounting. Journal of the Experimental Analysis of Behavior, 77(2), 129-146.

King, G. R., \& Logue, A. W. (1990). Choice in a self-control paradigm: effects of reinforcer quality. Behavioural Processes, 22, 89-99.

Kirby, K. N., \& Herrnstein, R. J. (1995). Preference reversals due to myopic discounting of delayed reward. Psychological Science, 6(2), 83-89.

Koob, G. F. (2000). Animal Models in Craving Research. Animal models of craving for ethanol. Addiction, 95(Supplement 2), S73-S81.

Logan, F. A. (1965). Decision making by rats: Uncertain outcome choices. Journal of Comparative and Physiological Psychology, 59(2), 246-251.

Logue, A. W., \& Pena-Correal, T. E. (1984). Responding during reinforcement delay in a self-control paradigm. Journal of the Experimental Analysis of Behavior, 41(3), 267-277.

Logue, A. W., Rodriguez, M. L., Pena-Correal, T. E., \& Mauro, B. C. (1984). Choice in a self-control paradigm: quantification of experience-based differences. Journal of the Experimental Analysis of Behavior, 41(1), 53-67.

Macey, D. J., Schulteis, G., Heinrichs, S. C., \& Koob, G. F. (1995). Time-dependent quantifiable withdrawal from ethanol in the rat: efect of method of induction. Alcohol, 13, 163-170.

Martinetti, M. P., Andrzejewski, M. E., Hineline, P., \& Lewis, M. J. (2000). Ethanol consumption and the matching law: a choice analysis using a limited-access paradigm. Experimental and Clinical Psychopharmacology, 8, 395-403.

Matthews, L. R., \& Temple, W. (1979). Concurrent schedule assessment of food preference in cows. Journal of the Experimental Analysis of Behavior, 32(2), 245-254.

Mazur, J., E. (1986). Choice between single and multiple delayed reinforcers. Journal of the Experimental Analysis of Behavior, 46(1), 67-77. 
Mazur, J., E. (1988). Estimation of indifference points with an adjusting-delay procedure. Journal of the Experimental Analysis of Behavior, 49(1), 37-47.

Mazur, J., E. (1994). Effects of intertrial reinforcers on self-control choice. Journal of the Experimental Analysis of Behavior, 61(1), 83-96.

Mazur, J., E. (2001). Hyperbolic value addition and general models of animal choice. Psychological Review, 108(1), 96-112.

Mazur, J., E., \& Kralik, J. D. (1990). Choice between delayed reinforcers and fixedratio schedules requiring forceful responding. Journal of the Experimental Analysis of Behavior, 53(1), 175-187.

Mazur, J., E., \& Logue, A. W. (1978). Choice in a "self-control" paradigm: effects of a fading procedure. Journal of the Experimental Analysis of Behavior, 30(1), 11-17.

McAdie, T. M., Foster, T. M., \& Temple, W. (1996). Concurrent schedules: quantifying the aversiveness of noise. Journal of the Experimental Analysis of Behavior, 65(1), 37-55.

McDiarmid, C. G., \& Rilling, M. E. (1965). Reinforcemen delay and reinforcement rate as determinants of schedule preference. Psychonomic Science, 2, 195-196.

McSweeney, F. K. (1978). Negative behavioral contrast on multiple treadle-press schedules. Journal of the Experimental Analysis of Behavior, 29(3), 463-473.

McSweeney, F. K., \& Melville, C. L. (1993). Behavioral contrast for key pecking as a function of component duration when only one component varies. Journal of the Experimental Analysis of Behavior, 60(2), 331-343.

McSweeney, F. K., Melville, C. L., \& Higa, J. (1988). Positive behavioral contrast across food and alcohol reinforcers. Journal of the Experimental Analysis of Behavior, 50, 469-481.

Miller, H. L., Jr. (1976). Matching-based hedonic scaling in the pigeon. Journal of the Experimental Analysis of Behavior, 26(3), 335-347.

Mitchell, S. H., \& Rosenthal, A. J. (2003). Effects of multiple delayed rewards on delay discounting in an adjusting amount procedure. Behavioural Processes, 64, 273-286.

Moore, J. (1982). Choice and multiple reinforcers. Journal of the Experimental Analysis of Behavior, 37(1), 115-122.

Myerson, J., \& Green, L. (1995). Discounting of delayed rewards: models of individual choice. Journal of the Experimental Analysis of Behavior, 64(3), 263-276.

Nowak, K. L., McKinzie, D. L., McBride, W. J., \& Murphy, J. M. (1999). Patterns of ethanol and saccarin intake in $\mathrm{P}$ rats under limited-access conditions. Alcohol, $19,85-96$. 
Peters, H. L., Hunt, M., \& Harper, D. N. (2004). Choice with initial and terminal link reinforcement: a alternative self-control paradigm. Journal of Experimental Psychology: Animal Behavior Processes, 30(1), 74-77.

Rachlin, H. (1971). On the tautology of the matching law. Journal of the Experimental Analysis of Behavior, 15(2), 249-251.

Rachlin, H. (1995). Self-control: beyond commitment. Behavioural and Brain Sciences, 18, 109-159.

Rachlin, H. (2000). The Science of Self-control. Cambridge, Massachusetts: Harvard University Press.

Rachlin, H., \& Green, L. (1972). Commitment, choice, and self-control. Journal of the Experimental Analysis of Behavior, 17(1), 15-22.

Rasmussen, D. D., Mitton, D. R., Green, J., \& Puchalski, S. (2001). Chronic daily ethanol and withdrawal: 2 . Behavioral changes during prolonged abstinence. Alcoholism: Clinical and Experimental Research, 25(7), 999-1005.

Reynolds, B., de Wit, H., \& Richards, J. B. (2002). Delay of gratifiction and delay discounting in rats. Behavioural Processes, 59, 157-168.

Robinson, J. K. (1992). Quantitative analyses of choice in rat and pigeons. The Psychological Record, 42, 437-445.

Samson, H. H. (1986). Initiation of ethanol reinforcement using a sucrose-substitution procedure in food- and water-sated rats. Alcoholism: Clinical and Experimental Research, 10(4), 436-442.

Samson, H. H. (2000). Animal Models in Craving Research: the microstructure of ethanol drinking: genetic and behavioral factors in the control of drinking patterns. Addiction, 95(Supplement 2), S61-S72.

Samson, H. H., Pfeffer, A. O., \& Tolliver, G. A. (1988). Oral ethanol selfadministration in rats: models of ethanol seeking behavior. Alcoholism: Clinical and Experimental Research, 12, 591-597.

Samson, H. H., Roehrs, T. A., \& Tolliver, G. A. (1982). Ethanol reinforced responding $i$ the rat: a concurrent analysis using sucrose as the alternate choice. Pharmacology, Biochemistry \& Behavior, 17, 333-339.

Schenk, S., Gorman, K., \& Amit, Z. (1990). Age-dependent effects of isolation housing on the self-administration of ethanol in laboratory rats. Alcohol, 7 , 321-326.

Snyderman, M. (1983). Delay and amount of reward in a concurrent chain. Journal of the Experimental Analysis of Behavior, 39(3), 437-447.

Sumpter, C. E., Foster, T. M., \& Temple, W. (1993). Predicting and scaling hens' preferences for topograhically different responses. Journal of the Experimental Analysis of Behavior, 63(2), 151-163. 
Trevett, A. J., Davison, M. C., \& Williams, R. J. (1972). Performance in concurrent interval schedules. Journal of the Experimental Analysis of Behavior, 17, 369374.

van Erp, A. M. M., \& Miczek, K. A. (2001). Persistent suppression of ethanol selfadministration by brief social stress in rats and increased startle response as index of withdrawal. Physiology \& Behavior, 73, 301-311.

Warry, C. J., Remington, B., \& Sonuga-Barke, E. J. S. (1999). When more means less: factors affecting human self-control in a local versus global choice paradigm. Learning and Motivation, 30(53-73).

Wearden, J. H., \& Burgess, I. S. (1982). Matching since Baum (1979). Journal of the Experimental Analysis of Behavior, 38(3), 339-348.

White, K. G., \& Alsop, B. (1993). Cognition in birds. In A. Sahgal (Ed.), Behavioural Neuroscience: A Practical Approach (pp. 137-147). Oxford: IRL Press.

White, K. G., \& Pipe, M. E. (1987). Sensitivity to reinforcer duration in a self-control procedure. Journal of the Experimental Analysis of Behavior, 48(2), 235-249.

Williams, B. A., \& Fantino, E. (1978). Effects on choice of reinforcement delay and conditioned reinforcement. Journal of the Experimental Analysis of Behavior, 29(1), 77-86.

Wolffgramm, J., Galli, G., Thimm, F., \& Heyne, A. (2000). Animal models of addiction: models for therapeutic strategies? Journal of Neural Transmission, 107, 649-668. 